University of South Florida

DIGITAL COMMONS

Digital Commons @ University of

@ UNIVERSITY OF SOUTH FLORIDA

South Florida

$11-16-2004$

\title{
Antenna Array Output Power Minimization Using Steepest Descent Adaptive Algorithm
}

Sandra Gomulka Johnson

University of South Florida

Follow this and additional works at: https://digitalcommons.usf.edu/etd

Part of the American Studies Commons

\section{Scholar Commons Citation}

Johnson, Sandra Gomulka, "Antenna Array Output Power Minimization Using Steepest Descent Adaptive Algorithm" (2004). USF Tampa Graduate Theses and Dissertations.

https://digitalcommons.usf.edu/etd/1098

This Dissertation is brought to you for free and open access by the USF Graduate Theses and Dissertations at Digital Commons @ University of South Florida. It has been accepted for inclusion in USF Tampa Graduate Theses and Dissertations by an authorized administrator of Digital Commons @ University of South Florida. For more information, please contact digitalcommons@usf.edu. 
Antenna Array Output Power Minimization Using Steepest Descent Adaptive Algorithm

by

Sandra Gomulka Johnson

A dissertation submitted in partial fulfillment of the requirements for the degree of

Doctor of Philosophy

Department of Electrical Engineering

College of Engineering

University of South Florida

Major Professor: Arthur David Snider, Ph.D.

Paul Flikkema, Ph.D.

Vijay K. Jain, Ph.D.

Nagarajan Ranganathan, Ph.D.

Mourad Ismail, Ph.D.

Date of Approval:

November 16, 2004

Keywords: signal processing, jammer nulling, GPS reception, exact gradient, Hilbert space gradient

(C) Copyright 2004, Sandra Gomulka Johnson 


\section{Dedication}

I dedicate this dissertation to my husband, William Joel Dietmar Johnson, and to my

daughter, Cora Julia Kimberly Johnson. It is through his generosity and motivation, her patience, and their love that this work has been accomplished. 


\section{Acknowledgments}

I would like to acknowledge my advisor, Dr. A. David Snider, for his patience, encouragement, and persistence during the course of our research. I must also acknowledge Dr. Paul Flikkema for introducing me to this research problem. I thank him as well as Chris Sperandio for their help with $\mathrm{AT}_{\mathrm{E}} \mathrm{X}$. The friendships of Carlos and Florence Briceno and the LaPointe family have been invaluable. Also I must include my parents, Richard and Rosemarie Gomulka, for their unwavering encouragement.

Finally, all thanks be to God from Whom all good things come. 


\section{Table of Contents}

List of Tables $\quad$ iii

List of Figures $\quad$ iv

Abstract $\quad$ V

Chapter 1 Introduction 1

1.1 Motivations 1

1.2 Antenna Array Fundamentals 2

Chapter $2 \quad$ Antenna Array Notation 5

Chapter 3 Existing Algorithms and Assessment Methodology: Comparison with the Oracle Solution $\quad 10$

3.1 Existing Adaptive Algorithms 11

3.1.1 Wiener Solution 11

3.1.2 Least Mean Square Solution 12

3.1.3 Power Inversion Solution 13

3.1.4 Subgradient Search Technique 13

3.2 A New Candidate: Hilbert-Space-Based (HSB) Gradient Algorithm 14

$\begin{array}{ll}3.3 \text { Algorithm Comparison Considerations } & 15\end{array}$

$\begin{array}{lll}3.3 .1 & \text { Oracle Solution } & 15\end{array}$

3.3.2 Condition Number 21

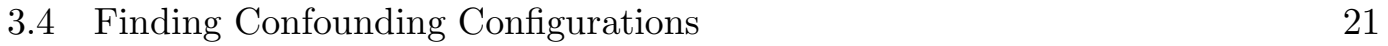

Chapter $4 \quad$ Hilbert-Space-Based (HSB) Gradient Algorithm 28

4.1 Power Minimization and Weight Calculation 28

4.2 Exact Gradient Calculation $\quad 32$

4.3 Noise Simulation 35

4.4 Differences in Algorithm and Oracle Solution 36

$\begin{array}{lll}\text { Chapter } 5 & \text { Matlab Implementation } & 38\end{array}$

$\begin{array}{lll}5.1 & \text { setup.m } & 38\end{array}$

5.2 adapt.m 40

5.3 motion.m 44

$\begin{array}{lll}\text { Chapter } 6 & \text { Algorithm and Oracle Performance } & 47\end{array}$

6.1 Summary of Performance $\quad 47$

6.1.1 No Motion 48 
$\begin{array}{lll}\text { 6.1.2 Yaw Motion } & 48\end{array}$

6.1.3 Full Motion $\quad 49$

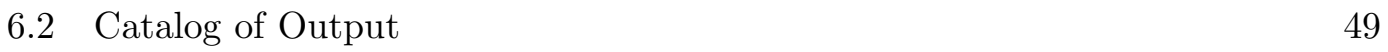

6.2.1 No Motion 49

6.2.2 Yaw Motion 51

6.2.3 Different Jammer Amplitude Levels 53

$\begin{array}{lll}6.2 .4 & \text { Full Motion } & 56\end{array}$

6.3 A Note on Out-of-Plane Jammers 58

6.4 Noise Immunity 58

$\begin{array}{lll}\text { Chapter } 7 & \text { Summary and Future Work Directions } & 61\end{array}$

$\begin{array}{ll}\text { References } & 62\end{array}$

$\begin{array}{ll}\text { Appendices } & 64\end{array}$

$\begin{array}{ll}\text { Appendix A } & \text { Symbol Glossary } \\ & 65\end{array}$

$\begin{array}{ll}\text { Appendix B } & \text { Matlab Source Code }\end{array}$

$\begin{array}{ll}\text { Appendix C } & \text { GPS Signal Characteristics }\end{array}$

Appendix D Alternative Gradient Formula 84

About the Author

End Page 


\section{List of Tables}

Table 1. Constants in setup.m.

Table 2. Variables in setup.m Initialized by User.

Table 3. Typical Data for No Motion Cases.

Table 4. Typical Data for Yaw Motion Cases.

Table 5. Typical Data for No Motion Cases with Different Jammer Amplitudes.

Table 6. Typical Data for Yaw Motion Cases with Different Jammer Amplitudes.

Table 7. Typical Data for Full Motion Cases.

Table 8. Algorithm Failure Rate with Increasing Noise Levels (noisefloor $=0 \mathrm{~dB}$ ).

Table 9. Algorithm Failure Rate with Increasing Noise Levels (noisefloor=1 dB).

Table 10. Algorithm Failure Rate with Increasing Noise Levels (noisefloor $=3 \mathrm{~dB}$ ). 


\section{List of Figures}

Figure 1. A 3 Element Linear Array.

Figure 2. Jammer Propagation Delay.

Figure 3. 3 Element Antenna Array with 2 Jammers.

Figure 4. Confounding Configuration Example.

Figure 5. Example of $\vec{a}_{m}$ and $\vec{u}_{n}$.

Figure 6. Six Jammers, No Motion.

Figure 7. Six Jammers, Yaw Motion, Near Confounding Configuration.

Figure 8. Four Jammers $50 \mathrm{~dB}, 30 \mathrm{~dB}, 40 \mathrm{~dB}, 20 \mathrm{~dB}$; No Motion.

Figure 9. Six Jammers 50, 30, 40, 20, 40, 50 dB; Yaw Motion.

Figure 10. Six Jammers 50, 30, 40, 20, 40, 50 dB; Full Motion. 


\title{
Antenna Array Output Power Minimization Using Steepest Descent Adaptive Algorithm
}

\author{
Sandra Gomulka Johnson
}

\begin{abstract}
A beamforming antenna array is a set of antennas whose outputs are weighted by complex values and combined to form the array output. The effect of the complex valued weights is to steer lobes and nulls of the array pattern to desired directions. These directions may be unknown and so the antenna weights must be adjusted adaptively until some measure of array performance is improved, indicating proper lobe or null placement.

An adaptive algorithm to adjust the complex weights of an antenna array is presented that nulls high power signals while allowing reception of GPS signals as long as the signals arrive from different directions. The GPS signals are spread spectrum modulated and have very low average power, on the order of background thermal noise. Simulations of the adaptive algorithm minimize the output power of the array to within $5 \mathrm{~dB}$ of the background noise level.

The adaptive algorithm, named the Hilbert-space-based (HSB) gradient method, is based on the steepest descent algorithm and implements an efficient, exact gradient calculation.

With $M$ antennas in the array, only $M-1$ weights are adjustable; one antenna weight is held constant to prevent the algorithm from minimizing the output power trivially by zeroing all weights thus preventing the reception of any signal by the array. It appears that $M-1$ adjustable antenna weights can null $M-1$ unwanted signals (jammers). However,
\end{abstract}


in the course of the algorithm development, a few configurations of antennas and jammer arrival directions were found where this is not true. Even when the jammer arrival directions are known ('oracle') certain configurations are mathematically impossible to cancel.

The oracle solution has a matrix formulation and under certain conditions an exact solution for antenna weights to annihilate the jammers can be found. This provides an excellent comparison tool to assess the performance of other adaptive algorithms.

The HSB gradient adaptive algorithm and the oracle solution are both implemented in Matlab. Outputs of both are plotted for comparison. 


\section{Chapter 1}

\section{Introduction}

\subsection{Motivations}

An antenna is a device to transmit and receive electromagnetic radiation. The antenna creates a pattern of radiation that spatially describes areas of gain (lobes) or attenuation (nulls) for the signals it transmits or receives. The radiation pattern of a single isotropic antenna is simpler than that of an array of multiple antennas; the radiation pattern of an array consists of a weighted superposition of each antenna's radiation pattern. The location of lobes and nulls in an array radiation pattern can be controlled with a beamforming network consisting of a signal processor implementing an algorithm that weights the outputs of each antenna and combines them to form the array output [9]. The subject of this dissertation is the signal processing algorithm. The RF data received by the antennas is assumed to be downconverted to a digital baseband stream and the signal processing constituting the proposed algorithm occurs completely in the digital domain.

The objective of the proposed Hilbert-space-based (HSB) gradient adaptive algorithm is to receive signals from the Global Positioning System (GPS) of satellites by nulling all jammer signals received by the array. The desired GPS signals have very low power, on the order of thermal noise, $-160 \mathrm{dBw}$, so if the output power of the array is above this level, it is assumed to come from jammer signals. The adaptive algorithm reduces the power output of the array by adapting weights of the antennas to null power. The adaptive algorithm is not based on forming lobes in the direction of arrival of the GPS signals.

The dissertation is organized as follows. The remainder of Chapter 1 introduces what a beamforming network is and includes an example to illustrate the effect of antenna weights on the array output. 
Chapter 2 introduces the vector notation used to describe the antenna array geometry and defines variables describing the input and output signals of the array.

Chapter 3 includes a survey of existing adaptive algorithms related to the HSB gradient algorithm and introduces the oracle solution. The oracle solution is a standard for assessing realistic solutions for antenna array weights based on array geometry and known jammer arrival directions.

Chapter 4 describes the theory of the HSB gradient algorithm including details of the exact gradient calculation and noise simulation.

Chapter 5 describes the implementation of the HSB gradient algorithm in Matlab.

Chapter 6 contains output of the Matlab simulation of the HSB gradient algorithm, illustrating its adaptive performance.

\subsection{Antenna Array Fundamentals}

This section will briefly describe the radiation pattern of an array of antennas and the effect the antenna weights have on this pattern.

The radiation pattern of an array is the superposition of the individual radiation patterns of each antenna in the array. The principle of pattern multiplication [1] states that the radiation pattern of an array of identical antennas is determined by the product of the element factor and the array factor. The element factor is the radiation pattern of an individual antenna in the array. The array factor depends on the geometry of the array antennas as well as on the complex weight of each antenna in the array. By adjusting the complex weights of the antennas in the array, the array factor and thus, the overall array radiation pattern, can be modified. Adaptive algorithms describe how to adjust the weights and a beamforming network implements these weight adjustments.

A beamforming network assigns an adjustable weight to each antenna output signal. Each weight is a complex number which has a variable gain (magnitude) and a variable phase. Adjusting the weight of each antenna modifies the array factor and has the effect of steering lobes and nulls to various positions. Signals arriving at the array in a null location will be severely attenuated or even eliminated by the array; signals arriving at a 
lobe will be enhanced by the array. Thus the antenna array performs spatial filtering using the mainlobe, sidelobes, and nulls of its radiation pattern. These are somewhat analogous to the passband, transition band, and stop band from traditional circuit filter theory. The spatial areas in the array pattern of gain and attenuation are frequency dependent. A deep null at $\omega_{1}$ may not be as deep, or even a null, at another frequency $\omega_{2}$. The bandwidths of the desired and jammer signals must be taken into account when designing the antenna array as well as the beamforming network. Each antenna weight in a narrowband array is a complex number; the antenna weights controlled by the proposed adaptive algorithm are implemented as such in the simulations to follow. For wideband arrays, the antenna weights must provide the ability to null jammers over a range of frequencies. These weights, being frequency dependent, can be implemented at each antenna as a linear filter or a digital tapped delay line [10]. General notation for a jammer impinging on an antenna array is developed in Chapter 2.

A simple example to illustrate the effect of gain and phase adjustments in a narrowband array follows. Consider a linear antenna array of 3 antennas each separated by a distance $r$ as in Figure 1. The signal arriving at the array is considered to be a plane wave modeled

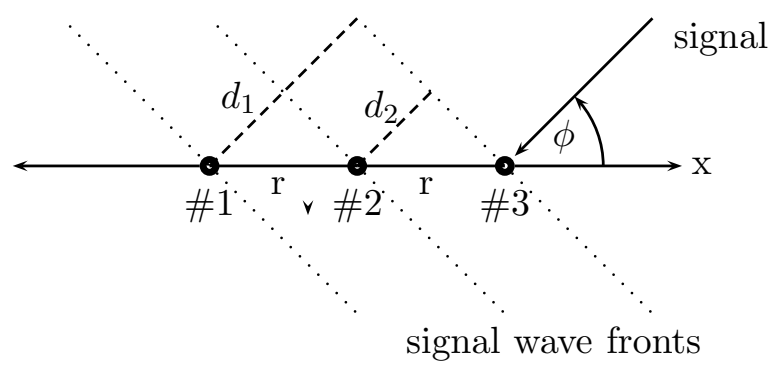

Figure 1. A 3 Element Linear Array.

as a sinusoid with frequency $\omega$ arriving at an angle $\phi$ with respect to the axis of the array. A particular wavefront of the signal will arrive at antenna \#3 first and after a delay of $\tau=d_{2} / c=r \cos \phi / c$ seconds ( $c$ is the propagation speed of the signal) the same wavefront of the signal will arrive at antenna \#2 and finally, after an additional delay of $\left(d_{1}-d_{2}\right) / c=r \cos \phi / c=\tau$ seconds, it arrives at antenna \#1. The output of the array is 


$$
w_{3} \cos \left(\omega t+q_{3}\right)+w_{2} \cos \left(\omega(t-\tau)+q_{2}\right)+w_{1} \cos \left(\omega(t-2 \tau)+q_{1}\right)
$$

where $w_{m}$ represents the gain of the $m$ th antenna and $q_{m}$ represents the phase shift introduced by the $m$ th antenna.

If the antenna phases are adjusted to compensate for the propagation delays so that the signal appears to have arrived at each antenna at the same time, the signal will appear to be boosted in amplitude at the output of the array. This is accomplished by adjusting the phase of antenna \#2 to be $q_{2}=\omega \tau$ and the phase of antenna \#1 to be $q_{1}=2 \omega \tau$ while keeping $w_{1}=w_{2}=w_{3}=1$. This results in the array output of $\cos (\omega t)+\cos (\omega(t-\tau)+$ $\omega \tau)+\cos (\omega(t-2 \tau)+2 \omega \tau)=3 \cos (\omega t)$.

The antenna weights can also be adjusted so that the array output is eliminated. With the antenna gains set to $w_{2}=-1 / 2$ and $w_{1}=-1 / 2$ and $q_{2}$ and $q_{1}$ defined as above, the output of the array is $\cos (\omega t)-\frac{1}{2} \cos \left(\omega(t-\tau)+q_{2}\right)-\frac{1}{2} \cos \left(\omega(t-2 \tau)+q_{1}\right)=\cos (\omega t)-$ $\frac{1}{2} \cos (\omega t)-\frac{1}{2} \cos (\omega t)=0$ and the signal has been eliminated at the output of the array.

These examples show that the choice of antenna weights can significantly affect the array output. Normally, it is up to the weight adjustment algorithm to distinguish friendly signals from jammer signals for proper lobe and null placement. The proposed algorithm does not distinguish the type of incoming signal although it assumes different power levels between the desired and unwanted signals; it simply tracks the output power of the array and adjusts the antenna weights until the output power is minimized. Adjusting antenna weights to achieve the desired array output when the input to the array is not well defined is the dilemma all adaptive algorithms must overcome, including the proposed algorithm to be described in Chapter 4. 


\section{Chapter 2}

\section{Antenna Array Notation}

This chapter will introduce the notation used to describe the configuration of antennas in an array as well as the input and output of the array. The geometry of the antenna array and the incoming signals will be expressed mathematically using vector notation. The incoming signals to the array are of 3 types: the GPS signals, the jammers, and noise. The GPS signals and noise are considered to have low power on the order of background noise levels, while the jammers are assumed to have a much higher power level. The basic strategy of the proposed HSB gradient algorithm focuses on reducing the array output jammer power to a level comparable to the output GPS signal power, so that the latter can be detected with spread-spectrum technology.

An array can consist of any number of antennas (assumed identical) arranged in any configuration. One antenna in the array is selected as the reference antenna. The origin of a reference coordinate system is chosen to coincide with the location of this reference antenna. The other antennas in the array are referred to as peripheral antennas. The antennas are located at positions $\vec{a}_{m}, m=1,2, \ldots, N_{\text {ant }}$ where $N_{\text {ant }}$ is the total number of antennas in the array. All peripheral antennas have adjustable gain $w_{m}$ and adjustable phase $q_{m}$ represented as a complex term $A_{m}=w_{m} e^{i q_{m}}$. The weight of the reference antenna, $m=1$, is fixed at a constant value, $A_{1} \equiv 1$.

The total GPS signal received by antenna $\# m$ is denoted $\chi_{G P S}^{(m)}(t)$. The number of individual GPS signals, each from a different GPS satellite, is not taken into account nor are their directions of arrival at the array.

The noise at antenna $\# m$ is $N_{m}(t)$. Details of the noise model used in the simulation are presented in Chapter 4 . 
The jammers, modeled as plane waves, arrive from directions $\vec{j}_{n}, n=1,2, \ldots, N_{\text {jam }}$, where $N_{j a m}$ is the total number of jammers impinging on the array, each with frequency $\omega_{n}$ and (complex) amplitude $J_{n}$. The phase of jammer $\# n$ at the reference antenna, $\varepsilon_{n}$, is incorporated into $J_{n}$. The $\vec{j}_{n}$ are unit vectors. A jammer signal received by the reference antenna as a sinusoid with frequency $\omega$ is (the real part of)

$$
J e^{i \omega t}=\left|J_{n}\right| e^{i\left(\omega t+\varepsilon_{n}\right)} .
$$

As stated previously in section 1.2, it takes a finite time for a jammer wavefront to propagate across the array and so the jammer wavefront reaches each peripheral antenna with a corresponding phase delay, with respect to the phase of the jammer wavefront at the reference antenna. These differences in propagation distance between the peripheral antennas and the reference antenna are given by $\vec{a}_{m} \bullet \vec{j}_{n}$, the projection of the location vector of antenna $\# m$ onto the vector representing the arrival direction of jammer \#n. Figure 2 shows a jammer arriving at a 3 element antenna array. The (negative) distance $x_{2}$ equals $\vec{a}_{2} \bullet \vec{j}_{1}$ and the distance $x_{3}$ equals $\vec{a}_{3} \bullet \vec{j}_{1}$. Each of these propagation distance differences

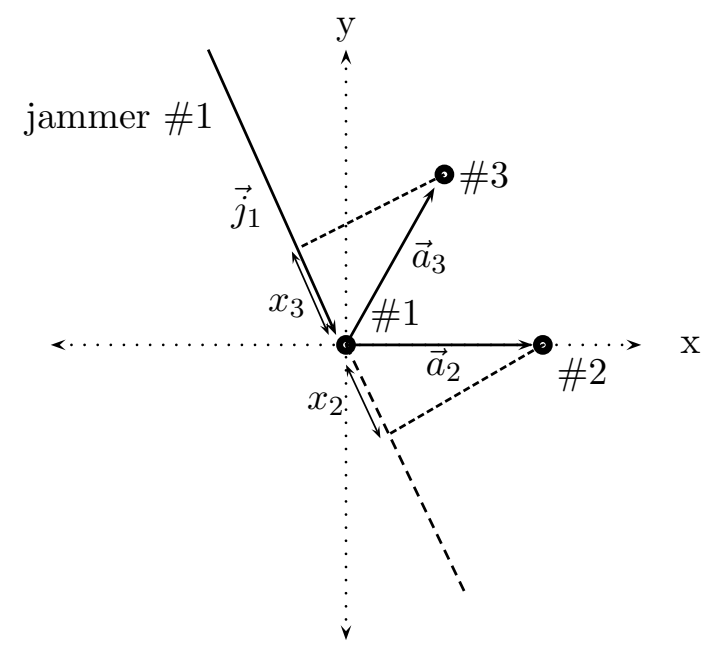

Figure 2. Jammer Propagation Delay. 
has an associated time delay of $\tau=\vec{a}_{m} \bullet \vec{j}_{n} / c$, where $c$ is the speed of propagation of the jammer signal (taken to be the speed of light), and a corresponding phase difference of

$$
\omega \tau=\frac{\omega\left(\vec{a}_{m} \bullet \vec{j}_{n}\right)}{c}=\frac{2 \pi f\left(\vec{a}_{m} \bullet \vec{j}_{n}\right)}{c}=\frac{2 \pi\left(\vec{a}_{m} \bullet \vec{j}_{n}\right)}{\lambda}
$$

where $\omega=2 \pi f$ and $\frac{f}{c}=\frac{1}{\lambda}$. From figure 2, the jammer wavefront reaches antenna \#3 $x_{3} / c$ seconds before it propagates to the reference antenna and the jammer propagates to antenna \#2 $x_{2} / c$ seconds after it has reached the reference antenna.

Each of the $m$ antennas in the array weights its input by $A_{m}$ and the array output is the sum of these weighted signals. The output signal at antenna $\# m$ consisting of a GPS signal, noise, and $N_{\text {jam }}$ jammers is then

$$
A_{m} \chi_{G P S}^{(m)}(t)+A_{m} N_{m}(t)+\sum_{n=1}^{N_{j a m}} J_{n} e^{i \omega_{n} t} e^{i \frac{2 \pi}{\lambda_{n}} \vec{a}_{m} \bullet \vec{j}_{n}} A_{m}
$$

The total output of the array, with $N_{j a m}$ jammers arriving at each antenna in the array, is the sum of the output of each antenna in the array

$$
\sum_{m=1}^{N_{a n t}} A_{m} \chi_{G P S}^{(m)}(t)+\sum_{m=1}^{N_{\text {ant }}} A_{m} N_{m}(t)+\sum_{n=1}^{N_{\text {jam }}} \sum_{m=1}^{N_{\text {ant }}} J_{n} e^{i \omega_{n} t} e^{i \frac{2 \pi}{\lambda_{n}} \vec{a}_{m} \bullet \vec{j}_{n}} A_{m}
$$

or in matrix form

$$
\begin{gathered}
{\left[\chi_{G P S}^{(1)}(t) \cdots \chi_{G P S}^{\left(N_{a n t}\right)}(t)\right]\left[\begin{array}{c}
A_{1} \\
A_{2} \\
\vdots \\
A_{N_{a n t}}
\end{array}\right]+\left[N_{1}(t) \cdots N_{N_{a n t}}(t)\right]\left[\begin{array}{c}
A_{1} \\
A_{2} \\
\vdots \\
A_{N_{a n t}}
\end{array}\right]} \\
+\left[J_{1} e^{i \omega_{1} t} J_{2} e^{i \omega_{2} t} \cdots J_{N_{j a m}} e^{i \omega_{N_{j a m}} t}\right]\left[\begin{array}{ccc}
e^{i \frac{2 \pi}{\lambda_{1}} \vec{a}_{1} \cdot \vec{j}_{1}} & \cdots & e^{i \frac{2 \pi}{\lambda_{1}} \vec{a}_{N_{a n t}} \cdot \vec{j}_{1}} \\
\vdots & \ddots & \vdots \\
e^{i \frac{2 \pi}{\lambda_{N_{j a m}}} \vec{a}_{1} \cdot \vec{j}_{N_{j a m}}} & \cdots & e^{i \frac{2 \pi}{\lambda_{N_{j a m}}} \vec{a}_{N_{a n t}} \cdot \vec{j}_{N_{j a m}}}
\end{array}\right]\left[\begin{array}{c}
A_{1} \\
A_{2} \\
\vdots \\
A_{N_{\text {ant }}}
\end{array}\right]
\end{gathered}
$$




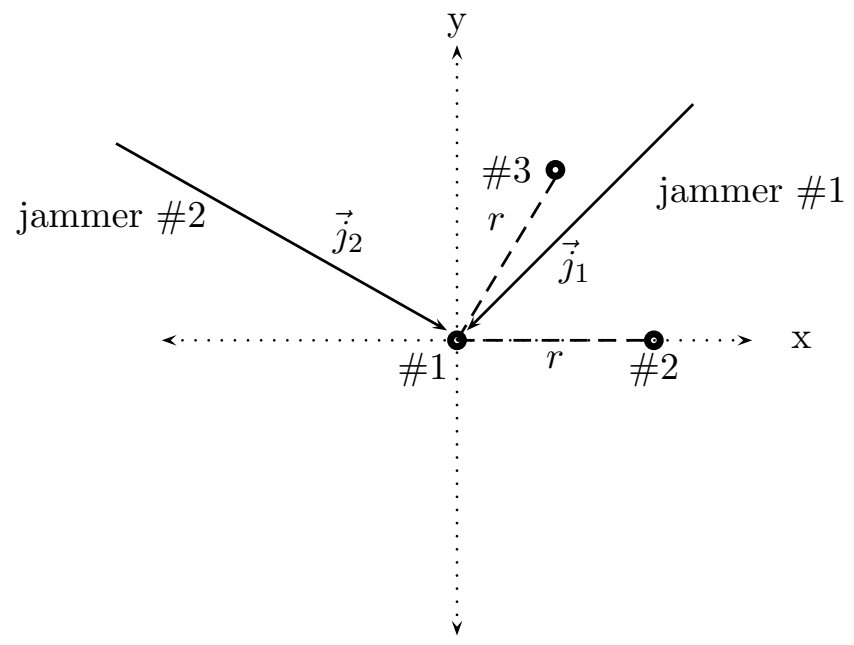

Figure 3. 3 Element Antenna Array with 2 Jammers.

$$
=\chi_{G P S}(t) A+N(t) A+J(t) C A=x(t) A
$$

where $x$ is the 'generic' input vector to the array, $\left(\chi_{G P S}^{(m)}(t)+N_{m}(t)+J(t) C\right)$, and $C$ is a matrix whose $(n, m)$ th element represents the phase difference of jammer $\# n$ arriving at antenna \#m

$$
C=\left[\begin{array}{cccc}
e^{i \frac{2 \pi}{\lambda_{1}} \vec{a}_{1} \cdot \vec{j}_{1}} & e^{i \frac{2 \pi}{\lambda_{1}} \vec{a}_{2} \cdot \vec{j}_{1}} & \cdots & e^{i \frac{2 \pi}{\lambda_{1}} \vec{a}_{N_{a n t}} \cdot \vec{j}_{1}} \\
e^{i \frac{2 \pi}{\lambda_{2}} \vec{a}_{1} \cdot \vec{j}_{2}} & e^{i \frac{2 \pi}{\lambda_{2}} \vec{a}_{2} \cdot \vec{j}_{2}} & \cdots & e^{i \frac{2 \pi}{\lambda_{2}} \vec{a}_{N_{a n t}} \cdot \vec{j}_{2}} \\
\vdots & \vdots & \ddots & \vdots \\
e^{i \frac{2 \pi}{\lambda_{N_{j a m}}} \vec{a}_{1} \cdot \vec{j}_{N_{j a m}}} & e^{i \frac{2 \pi}{\lambda_{N_{j a m}}} \vec{a}_{2} \cdot \vec{j}_{N_{j a m}}} & \cdots & e^{i \frac{2 \pi}{\lambda_{N_{j a m}}} \vec{a}_{N_{a n t}} \cdot \vec{j}_{N_{j a m}}}
\end{array}\right]
$$

A simple example will illustrate the vector notation just introduced. Consider a 3 element planar array with the reference antenna located at the origin $\left(\vec{a}_{1}=0\right)$ and peripheral antennas located at $\vec{a}_{2}=r \hat{i}, \vec{a}_{3}=\frac{1}{2} r \hat{i}+\frac{\sqrt{3}}{2} r \hat{j}$ as in figure 3 . The arrival directions of two jammers are $\vec{j}_{1}=\frac{1}{\sqrt{2}} \hat{i}+\frac{1}{\sqrt{2}} \hat{j}$ and $\vec{j}_{2}=-\frac{\sqrt{3}}{2} \hat{i}+\frac{1}{2} \hat{j}$. 
The dot products of the antenna vectors and jammer vectors are

$$
\begin{aligned}
& \vec{a}_{1} \bullet \vec{j}_{1}=0 \\
& \vec{a}_{2} \bullet \vec{j}_{1}=\frac{r}{\sqrt{2}} \\
& \vec{a}_{3} \bullet \vec{j}_{1}=\left(\frac{1}{2 \sqrt{2}}+\frac{\sqrt{3}}{2 \sqrt{2}}\right) r \\
& \vec{a}_{1} \bullet \vec{j}_{2}=0 \\
& \vec{a}_{2} \bullet \vec{j}_{2}=-\frac{\sqrt{3}}{2} r \\
& \vec{a}_{3} \bullet \vec{j}_{2}=0
\end{aligned}
$$

and the array output is

$$
\begin{aligned}
& {\left[\chi_{G P S}^{(1)}(t) \chi_{G P S}^{(2)}(t) \chi_{G P S}^{(3)}(t)\right]\left[\begin{array}{c}
A_{1} \\
A_{2} \\
A_{3}
\end{array}\right]+\left[N_{1}(t) N_{2}(t) N_{3}(t)\right]\left[\begin{array}{c}
A_{1} \\
A_{2} \\
A_{3}
\end{array}\right]} \\
& +\left[J_{1} e^{i \omega_{1} t} J_{2} e^{i \omega_{2} t}\right]\left[\begin{array}{ccc}
1 & e^{i \frac{2 \pi}{\lambda} r / \sqrt{2}} & e^{i \frac{2 \pi}{\lambda} r(1+\sqrt{3}) / 2 \sqrt{2}} \\
1 & e^{-i \frac{2 \pi}{\lambda} r \sqrt{3} / 2} & 1
\end{array}\right]\left[\begin{array}{c}
A_{1} \\
A_{2} \\
A_{3}
\end{array}\right] \text {. }
\end{aligned}
$$

Since the array output is dominated by the high power jammers, the task of the proposed algorithm is to adjust the antenna weights, $A_{2}, \ldots, A_{N_{a n t}}$, so that nulls are steered toward the jammer arrival directions, minimizing the jammer power to the level of the noise and GPS signals. This leaves the GPS signals intact to be processed by spread spectrum demodulation techniques. Recall the reference antenna weight is fixed at $A_{1}=1$ so that the algorithm minimizes the output power while avoiding the trivial solution, $A_{m}=0, m=1,2, \ldots, N_{\text {ant }}$.

Note that even though the jammer arrival directions appear explicitly in the formulation just presented, in practice one does not know the jammer arrival directions or frequencies. At this point of the exposition, the inclusion of the jammer arrival directions is necessary to formulate the phase differences between the jammers and the antennas as the jammers propagate across the array. 


\section{Chapter 3}

\section{Existing Algorithms and Assessment Methodology: Comparison with the Oracle Solution}

Adaptive algorithms are used in a wide variety of applications. When used with an antenna array, the adaptive algorithm modifies the antenna weights based on some cost function. When the cost function indicates improved array performance, the algorithm is adapting the antenna weights in the desired fashion. The choice of algorithm to adaptively adjust the antenna weights is determined by such factors as the performance characteristics to be improved, known information about the operating environment of the array, and cost of implementation.

This chapter will provide a review of existing techniques used by adaptive algorithms. The proposed algorithm is related to and shares similarities with these existing algorithms. Also, this chapter contains details of the oracle solution. This is an exact solution for antenna weights based on minimizing jammer output power with knowledge of the jammer

parameters. It is called the 'oracle' because the jammer information is unavailable in practice. The oracle provides a metric upon which the performance of other adaptive algorithms can be assessed.

A direct comparison of antenna weights between one algorithm and another is not always possible. After the first iteration of each algorithm, the weights evolve along different trajectories. This chapter will introduce the scenario that an array may encounter certain configurations which cause the algorithm to stall. The oracle solution is a history-free standard assessing how an algorithm is doing at a particular configuration, regardless of how it got there. 


\subsection{Existing Adaptive Algorithms}

\subsubsection{Wiener Solution}

A popular cost function that is used in adaptive array algorithms is the mean squared error [10]. The error is a measure between some reference or desired array output signal, $d$, and the actual array output signal, $x(t) A$ where $A$ is a vector of antenna weights and $x(t)$ is the vector of antenna input signals (assumed real for this exposition). The error signal is

$$
e(t)=d(t)-x(t) A
$$

and the mean squared error is

$$
\operatorname{MSE}=E\left[e^{2}(t)\right]=d^{2}(t)-2 E[d(t) x(t)] A+A^{T} E\left[x^{T}(t) x(t)\right] A .
$$

The term $E[d(t) x(t)]=r_{x d}$ is the cross-correlation vector of the actual input and the desired signal and the term $E\left[x^{T}(t) x(t)\right]=R_{x x}$ is the autocorrelation matrix of the actual input signal. The MSE is a quadratic function of the antenna weights and can be visualized as a surface with (typically) a unique minimum. The weights correspond to some point on the MSE surface and those that correspond to the minimum of this surface are those that are sought by the adaptive algorithm. A way to approach the minimum is by adjusting the weight values in the direction of the negative gradient, $-\nabla$, on this surface

$$
A_{\text {new }}=A_{\text {old }}-\mu \nabla
$$

where $\mu$ is a constant that determines the fraction of the gradient to be implemented per weight adjustment. The gradient of the MSE (assuming real elements of $A$ ) is

$$
\nabla=\frac{\partial E\left[e^{2}(t)\right]}{\partial A}=2 r_{x d}+2 R_{x x} A
$$


The gradient has a value of zero at the minimum point on the surface. Setting the gradient to zero and solving for $A$ yields the Wiener solution for the antenna weights,

$$
A=-R_{x x}^{-1} r_{x d}
$$

This solution, however, requires the statistics of the input signal to be known, i.e., $r_{x d}$ and $R_{x x}$. This is rare in practice so, an alternative to the Wiener solution is proposed herein.

\subsubsection{Least Mean Square Solution}

An alternative to the Wiener solution involves the instantaneous value of the gradient on the squared error surface [11]. The squared error is

$$
e^{2}(t)=(d(t)-x(t) A)^{2}
$$

and its gradient is

$$
\nabla\left(e^{2}(t)\right)=\frac{\partial e^{2}(t)}{\partial A}=-2 e(t) x(t)
$$

This is only an approximation to the Mean Squared Error gradient because the instantaneous values of the error and input signals, instead of their exact statistics as in the Wiener solution, are used in the gradient calculation. The weight update equation becomes

$$
A_{\text {new }}=A_{\text {old }}+\mu(2 e(t) x(t)) .
$$

This is usually called the least mean square (LMS) algorithm. Despite the name of this algorithm, the LMS does not seek the least mean squared error but rather the least squared error in the solution for $A$. As stated previously, the squared error is a quadratic function of the weights and can typically be interpreted as a surface with a unique minimum. The LMS algorithm approaches this minimum with iterative weight adjustments in the negative gradient direction. The weight adjustments continue until the gradient is zero or until 
further weight adjustments no longer reduce the squared error. At this point, the array output due to these weight values approximates the desired signal with a least squared error.

\subsubsection{Power Inversion Solution}

Compton has proposed an algorithm whose objective is to minimize the output power of an antenna array and it is called the power-inversion algorithm for adaptive arrays [15]. It improves the signal to interference ratio in an adaptive array and is based on the HowellsApplebaum feedback [12] to minimize array output power. The arrival directions of the desired signals and interference signals need not be known and the best performance occurs when the desired signal is near thermal noise levels. The power-inversion algorithm requires the designer to choose a value for loop gain which depends on three things: 1) the required minimum signal-to-noise ratio (SNR) out of the array, 2) the dynamic range of the signal level that the array must accommodate, and 3) what signals the array is receiving: the desired signal only, the desired and interference signals only, or desired, interference and noise signals. The optimal weights are calculated with an equation dependent on the desired signal power per element, interference power per element, desired SNR per element, and

interference-to-noise ratio per element. This algorithm requires a substantial amount of information about the array's operating environment before effective antenna weights can be calculated.

\subsubsection{Subgradient Search Technique}

Another antenna array power minimization method uses a so-called "subgradient" technique to search a performance surface for the optimal antenna weights [6]. This method assumes an array containing a fixed-weight reference antenna with the remaining antennas, referred to as peripheral antennas, having adjustable weights. The technique sequentially sets the peripheral antenna weights to values from a known, finite list of settings, based on the locations of the antenna elements, until the output power of the array is minimized. The jammers are viewed as phasors whose magnitudes and phases are manipulated by the 
peripheral antenna weights. The algorithm adjusts the peripheral antenna weights until the overall jammer phasor from the peripheral antennas cancels or minimizes the jammer phasor from the reference antenna whose weight is fixed.

As an example, consider a planar array in the $x y$-plane with $N_{\text {ant }}=7$ antennas, 6 peripheral antennas equally spaced around a central reference antenna. Let the origin of the $x y$ axes coincide with the central antenna. The subgradient search sets the peripheral antenna weights to the values from the set $\left\{\frac{1}{N_{a n t}-1} e^{i\left(\pi-\phi_{m}\right)}, m=2,3, \ldots, N_{\text {ant }}\right\}$, where $\phi_{m}$ is the angle describing the location of the $m$ th peripheral antenna measured counterclockwise from the $x$-axis. The finite list of settings comprise these values. The technique monitors the output power of the array as each antenna weight takes on each value from the finite set. When all combinations have been tried, the technique chooses the optimal weights as those giving the lowest output power.

The results of this technique were compared in [6] to results of the LMS algorithm for a 4 element planar array receiving GPS and jammer signals. The LMS algorithm outperformed the simple, computationally inexpensive, subgradient technique in static and dynamic cases (roll motion) but with the tradeoff of more complex hardware implementation. Although the subgradient technique was outperformed by the LMS algorithm, it still provided sufficient nulling for successful GPS signal detection.

\subsection{A New Candidate: Hilbert-Space-Based (HSB) Gradient Algorithm}

The algorithms mentioned above, LMS, Wiener, and Compton, are powerful algorithms when information is known about the array operating environment, and about the input and desired signal characteristics. The desired signal must be approximated in practice and any error in this approximation will propagate to the weight solution. The desired signal for the HSB gradient algorithm to be described in Chapter 4 is a constant (zero); the algorithm adjusts the antenna weights until the output power is minimized.

The statistics of the input signal are rarely known for all potential inputs to the antenna array and the problem is further compounded when the input data is not stationary. The HSB gradient algorithm works successfully with nonstationary data and the dynamic 
range of the input signals and output SNR need not be known for successful algorithm performance.

The HSB gradient algorithm efficiently calculates the exact gradient on the performance surface based on a time-averaged power output of an array instead of approximating a gradient with instantaneous signal statistics. The HSB gradient algorithm weights are not limited to a finite set of possible values as are the weights from the subgradient search technique. Therefore, the performance of the HSB gradient algorithm is expected to exceed that of the subgradient search technique.

\subsection{Algorithm Comparison Considerations}

Given the variety of adaptive algorithms that are available, one would like to know which algorithm will result in the best performance for a given application. Comparisons among the outputs of a group of potential algorithms are a way to select the best performing algorithm but this requires that all potential algorithms be implemented, to obtain the outputs to make such comparisons. Therefore, comparing any number of different algorithms can be a very time consuming task.

All algorithms seek a solution that minimizes some measure of the array output without knowledge of jammer parameters. This section introduces the 'oracle' solution which seeks to zero the array output with knowledge of the jammer parameters. The oracle thus provides a standard against which other algorithms can be measured. If there is no oracle solution, then no other algorithm can possibly succeed in nulling the output for the given jammer parameters.

\subsubsection{Oracle Solution}

In the course of simulating the HSB gradient algorithm described in Chapter 4 with an array in motion (array motion is simulated with roll, pitch, and yaw), occasionally a configuration of the antennas of the array and the jammers was encountered that confounded the convergence of the algorithm, and the output power was not minimized. The array appeared to move through such regions of divergence and then return to regions of conver- 
gence, indicating that the motion of the array through certain jammer arrival configurations caused the divergence of the algorithm.

This opened a new question as to whether it was possible to formulate configurations of antennas and jammers that were mathematically unsolvable. If such configurations could be found and characterized, the configurations that confounded the HSB gradient algorithm might be explained.

The existence of a solution for antenna weights to null the jammers, presuming known antenna locations and known jammer directions, called the "oracle solution", was studied. The configurations of antennas and jammers for which no oracle solution exists were named "confounding configurations".

The oracle solution for the antenna weights will be described here. Recall the output of the array from equation (5) is $\chi_{G P S} A+N A+J C A$. The goal of any adaptive algorithm is to minimize the power in this array output. Consider, instead, the task of forcing only the jammer component of the output to be zero, i.e., $J C A=0$. Since the jammer amplitude vector, $J$, cannot be controlled, the oracle cannot exploit any feature about the amplitudes of the jammers, and thus it must solve

$$
C A=0
$$

(for $A$ ). The jammer amplitudes do not affect the values of the oracle antenna weights; they provide no information for these weights. The arrival directions of the jammers $\vec{j}_{n}$, however, directly influence the solution. They appear in the elements of $C$ :

$$
C A=\left[\begin{array}{cccc}
e^{i \frac{2 \pi}{\lambda} \vec{a}_{1} \cdot \vec{j}_{1}} & e^{i \frac{2 \pi}{\lambda_{1}} \vec{a}_{2} \cdot \vec{j}_{1}} & \cdots & e^{i \frac{2 \pi}{\lambda_{1}} \vec{a}_{N_{a n t}} \cdot \vec{j}_{1}} \\
e^{i \frac{2 \pi}{\lambda_{2}} \vec{a}_{1} \cdot \vec{j}_{2}} & e^{i \frac{2 \pi}{\lambda_{2}} \vec{a}_{2} \cdot \vec{j}_{2}} & \cdots & e^{i \frac{2 \pi}{\lambda_{2}} \vec{a}_{N_{a n t}} \cdot \vec{j}_{2}} \\
\vdots & \vdots & \ddots & \vdots \\
e^{i \frac{2 \pi}{\lambda_{N_{j a m}}} \vec{a}_{1} \cdot \vec{j}_{N_{j a m}}} & e^{i \frac{2 \pi}{\lambda_{N_{j a m}}} \vec{a}_{2} \cdot \vec{j}_{N_{j a m}}} & \cdots & e^{i \frac{2 \pi}{\lambda_{N_{j a m}}} \vec{a}_{N_{\text {ant }}} \cdot \vec{j}_{N_{j a m}}}
\end{array}\right]\left[\begin{array}{c}
A_{1} \\
A_{2} \\
\vdots \\
A_{N_{\text {ant }}}
\end{array}\right]=\left[\begin{array}{c}
0 \\
0 \\
\vdots \\
0
\end{array}\right] .
$$


All elements of the first column of $C$ have terms which contain $\vec{a}_{1} \bullet \vec{j}_{n}$ and since $\vec{a}_{1}=0$ for the reference antenna the first column is a column of 1 's. Also, $A_{1} \equiv 1$ for the reference antenna. Equation (7) can thus be rewritten as

$$
\left[\begin{array}{cccc}
1 & e^{i \frac{2 \pi}{\lambda} \vec{a}_{2} \cdot \vec{j}_{1}} & \cdots & e^{i \frac{2 \pi}{\lambda_{1}} \vec{a}_{N_{a n t}} \cdot \vec{j}_{1}} \\
1 & e^{i \frac{2 \pi}{\lambda_{2}} \vec{a}_{2} \cdot \vec{j}_{2}} & \cdots & e^{i \frac{2 \pi}{\lambda_{2}} \vec{a}_{N_{a n t}} \cdot \vec{j}_{2}} \\
\vdots & \vdots & \ddots & \vdots \\
1 & e^{i \frac{2 \pi}{\lambda_{N_{j a m}}} \vec{a}_{2} \cdot \vec{j}_{N_{j a m}}} & \cdots & e^{i \frac{2 \pi}{\lambda_{N_{j a m}}} \vec{a}_{N_{a n t}} \cdot \vec{j}_{N_{j a m}}}
\end{array}\right]\left[\begin{array}{c}
1 \\
A_{2} \\
\vdots \\
A_{N_{a n t}}
\end{array}\right]=\left[\begin{array}{c}
0 \\
0 \\
\vdots \\
0
\end{array}\right]
$$

These equations can be further rearranged by taking the first column of $C$ to the right hand side. The $C$ matrix with the first column removed is now referred to as $C_{\text {reduced }}$ with size $N_{\text {jam }} \times\left(N_{\text {ant }}-1\right)$.

$$
\left[\begin{array}{ccc}
e^{i \frac{2 \pi}{\lambda_{1}} \vec{a}_{2} \cdot \vec{j}_{1}} & \cdots & e^{i \frac{2 \pi}{\lambda_{1}} \vec{a}_{N_{\text {ant }}} \cdot \vec{j}_{1}} \\
e^{i \frac{2 \pi}{\lambda_{2}} \vec{a}_{2} \cdot \vec{j}_{2}} & \cdots & e^{i \frac{2 \pi}{\lambda_{2}} \vec{a}_{N_{a n t}} \cdot \vec{j}_{2}} \\
\vdots & \ddots & \vdots \\
e^{i \frac{2 \pi}{\lambda_{N_{j a m}}} \vec{a}_{2} \cdot \vec{j}_{N_{j a m}}} & \cdots & e^{i \frac{2 \pi}{\lambda_{N_{\text {jam }}}} \vec{a}_{N_{\text {ant }}} \cdot \vec{j}_{N_{\text {jam }}}}
\end{array}\right]\left[\begin{array}{c}
A_{2} \\
\vdots \\
A_{N_{\text {ant }}}
\end{array}\right]=C_{\text {reduced }}\left[\begin{array}{c}
A_{2} \\
\vdots \\
A_{N_{\text {ant }}}
\end{array}\right]=\left[\begin{array}{c}
-1 \\
-1 \\
\vdots \\
-1
\end{array}\right] .
$$

A criterion for the existence of an oracle solution can be stated as:

Antenna weights annihilating the jammer output power exist if and only if the vector $[-1-1 \cdots-1]^{T}$ lies in the column space of $C_{\text {reduced }}$.

When the number of jammers equals the number of peripheral antennas, $N_{\text {jam }}=N_{\text {ant }}-$ $1, C_{\text {reduced }}$ is square. If, further, $\operatorname{rank}\left(C_{\text {reduced }}\right)=N_{\text {ant }}-1$, the unique exact antenna weights 
are

$$
\left[\begin{array}{c}
A_{2} \\
A_{3} \\
\vdots \\
A_{N_{\text {ant }}}
\end{array}\right]=-C_{\text {reduced }}^{-1}\left[\begin{array}{c}
1 \\
1 \\
\vdots \\
1
\end{array}\right]
$$

If the number of jammers is less than the number of peripheral antennas, $N_{j a m}<$ $N_{\text {ant }}-1$, the system of equations (9) is underdetermined. In this case, if the rank of $C_{\text {reduced }}$ is full, $\operatorname{rank}\left(C_{\text {reduced }}\right)=N_{\text {jam }}$, the system has an infinite number of solutions. For the case when there are more jammers than peripheral antennas, $N_{j a m}>N_{a n t}-1$, the array is asked to form $N_{\text {jam }}$ nulls with only $N_{a n t}-1<N_{\text {jam }}$ adjustable antenna weights. This results in an overdetermined system of equations (9) with the possibility-or rather, likelihood-of no solution for the antenna weights.

(Of course a least squares solution to equation (9) of minimum norm [2] can always be expressed using the pseudoinverse of $C_{\text {reduced }}[3]$, written as $C_{\text {reduced }}^{-\psi}$, as

$$
\left.\left[\begin{array}{c}
A_{2} \\
A_{3} \\
\vdots \\
A_{N_{\text {ant }}}
\end{array}\right]=-C_{\text {reduced }}^{-\psi}\left[\begin{array}{c}
1 \\
1 \\
\vdots \\
1
\end{array}\right] .\right)
$$

The following is a simple example of an $N_{\text {ant }}=3$ antenna array receiving $N_{\text {jam }}=2$ jammers, $\left(N_{\text {jam }}=N_{\text {ant }}-1\right)$. With 2 adjustable antenna weights, it seems reasonable to expect that 2 nulls can be formed to attenuate 2 jammers. The example will illustrate that this is not always true.

The antennas are located at $\vec{a}_{1}=0, \vec{a}_{2}=r \hat{i}, \vec{a}_{3}=\frac{1}{2} r \hat{i}+\frac{\sqrt{3}}{2} r \hat{j}$ and the jammer arrival directions are $\vec{j}_{1}=\frac{\sqrt{3}}{2} \hat{i}+\frac{1}{2} \hat{j}$ and $\vec{j}_{2}=-\frac{\sqrt{3}}{2} \hat{i}-\frac{1}{2} \hat{j}$, see figure 4 . The jammer frequencies are 


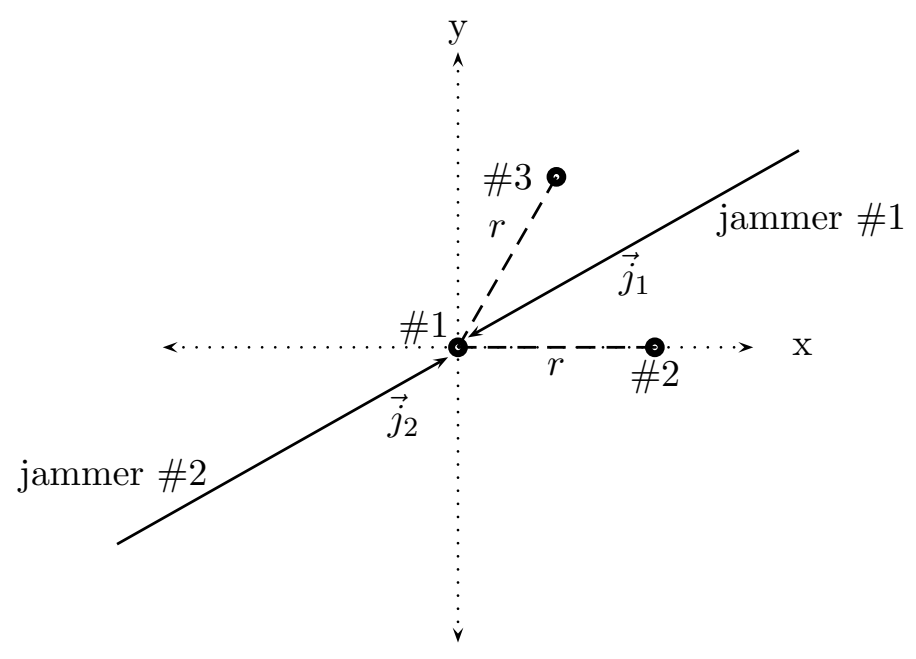

Figure 4. Confounding Configuration Example.

the same, $\omega_{1}=\omega_{2}$. The antenna weights will null the output, from equation (8), if

$$
\left[\begin{array}{ccc}
1 & e^{i \frac{2 \pi}{\lambda} \vec{a}_{2} \cdot \vec{j}_{1}} & e^{i \frac{2 \pi}{\lambda} \vec{a}_{3} \cdot \vec{j}_{1}} \\
1 & e^{i \frac{2 \pi}{\lambda} \vec{a}_{2} \cdot \vec{j}_{2}} & e^{i \frac{2 \pi}{\lambda} \vec{a}_{3} \cdot \vec{j}_{2}}
\end{array}\right]\left[\begin{array}{c}
1 \\
A_{2} \\
A_{3}
\end{array}\right]=\left[\begin{array}{l}
0 \\
0 \\
0
\end{array}\right] .
$$

Substituting in the values for $\vec{a}_{m}$ and $\vec{j}_{n}$,

$$
C_{\text {reduced }}=\left[\begin{array}{cc}
e^{i \frac{2 \pi}{\lambda} \frac{\sqrt{3}}{2} r} & e^{i \frac{2 \pi}{\lambda} \frac{\sqrt{3}}{2} r} \\
e^{-i \frac{2 \pi}{\lambda} \frac{\sqrt{3}}{2} r} & e^{-i \frac{2 \pi}{\lambda} \frac{\sqrt{3}}{2} r}
\end{array}\right]
$$

Let $e^{i \beta}=e^{i \frac{2 \pi}{\lambda} \frac{\sqrt{3}}{2} r}$ and equation (9) becomes

$$
\left[\begin{array}{cc}
e^{i \beta} & e^{i \beta} \\
e^{-i \beta} & e^{-i \beta}
\end{array}\right]\left[\begin{array}{l}
A_{2} \\
A_{3}
\end{array}\right]=\left[\begin{array}{c}
-1 \\
-1
\end{array}\right]
$$


Rewrite the left hand side of this equation as

$$
A_{2}\left[\begin{array}{c}
e^{i \beta} \\
e^{-i \beta}
\end{array}\right]+A_{3}\left[\begin{array}{c}
e^{i \beta} \\
e^{-i \beta}
\end{array}\right]=\left(A_{2}+A_{3}\right)\left[\begin{array}{c}
e^{i \beta} \\
e^{-i \beta}
\end{array}\right]
$$

But the right hand side of the equation is

$$
\left[\begin{array}{l}
-1 \\
-1
\end{array}\right]
$$

$\left[e^{i \beta} e^{-i \beta}\right]^{T}$ is a scalar multiple of $[-1-1]^{T}$ if and only if $e^{i \beta}=e^{-i \beta}$, that is, $e^{i 2 \beta}=1, \beta=k \pi$, where $k$ is an integer. Thus, these jammers can be nulled only if $\beta=\frac{2 \pi}{\lambda} \frac{\sqrt{3}}{2} r=k \pi$, that is, if the jammer wavelength $\lambda$ equals $\sqrt{3}$ times the antenna separation, $r$, divided by an integer, i.e.

$$
\lambda=\sqrt{3} r / k
$$

For this example, even though the number of jammers is equal to the number of peripheral antennas in the array, $N_{\text {jam }}=N_{\text {ant }}-1$, making $C_{\text {reduced }}$ a square matrix, if the jammer wavelength does not satisfy equation (12) the vector $\left[\begin{array}{ll}-1 & -1\end{array}\right]^{T}$ does not lie in the column space of $C_{\text {reduced }}$. The jammer power, therefore, cannot be nulled for this configuration.

We propose that whenever a prescribed configuration of jammers and antennas causes any adaptive power-minimizing algorithm to diverge, the configuration should be tested for an oracle solution before the algorithm is "blamed". If the configuration has no oracle solution, it should be removed from the test set in assessing the performance of the adaptive algorithm, because no algorithm could possibly give satisfactory performance.

Whenever an oracle solution does exist, on the other hand, it completely nulls the jammer power. Thus, it provides a standard against which other algorithms can be measured.

To summarize, if an adaptive algorithm fails to perform as expected, the oracle solution analysis can perhaps provide a potential clue by exposing a confounding configuration. The oracle solution, if it exists, also provides an evaluation of the effectiveness of an adaptive algorithm solution. 


\subsubsection{Condition Number}

When no oracle solution exists, the configuration of jammer arrival directions and antenna locations is labeled a confounding configuration. However, there exist configurations of jammers and antennas for which there is an oracle solution but it is numerically unstable; these such cases are called near-confounding configurations. A metric used during research of confounding configurations was the condition number of $C_{\text {reduced }}$ which tests the numerical robustness of an oracle solution. The condition number measures sensitivity to error [16] and it is calculated by taking the ratio of the largest singular value of the matrix to the smallest singular value. The higher the condition number of a matrix, the closer the matrix is to being singular [16]; a condition number of infinity indicates a matrix that is singular, one without full rank.

A confounding configuration can result from a singular $C_{\text {reduced }}$. For cases with $N_{j a m}=$

$N_{\text {ant }}-1$ ( $C_{\text {reduced }}$ square), if the condition number of $C_{\text {reduced }}$ is large, on the order of $1 \times 10^{4}$, the arrangement of jammers and antennas may confound the HSB gradient algorithm and is labeled a near-confounding configuration.

Therefore, the condition number of $C_{\text {reduced }}$ provides a useful indication as to whether any adaptive algorithm will be able to find optimal weights to null jammers. If the condition number is on the order of $1 \times 10^{4}$ or greater the HSB gradient algorithm may not produce viable weights and the antenna/jammer configuration producing the high condition number should be removed from the test set.

\subsection{Finding Confounding Configurations}

The previous section described how the oracle can be used to test whether given antenna/jammer configurations are confounding or near-confounding. This section examines some aspects of the oracle solution, exploring the ability to construct confounding antenna/jammer configurations. This may be useful for jamming an enemy when the configuration of the enemy's array is known. 
The exact antenna weights $A$ to annihilate incident jammers, the oracle solution, exist if the vector $[-1-1 \cdots-1]^{T}$ lies in the column space of $C_{\text {reduced }}$

$$
C_{\text {reduced }}\left[\begin{array}{c}
A_{2} \\
A_{3} \\
\vdots \\
A_{N_{\text {ant }}}
\end{array}\right]=\left[\begin{array}{c}
-1 \\
-1 \\
\vdots \\
-1
\end{array}\right] \text {. }
$$

In this section, it is desired to find jammer arrival directions that defy an oracle solution, i.e., confounding configurations. One way, among many, to explore this starts with introducing the variable

$$
\vec{v}_{n}=\frac{2 \pi}{\lambda} \vec{j}_{n}
$$

for more compact notation and factors the matrix $C_{\text {reduced }}$ as

$$
C_{\text {reduced }}=C_{\text {reduced }}^{\prime} \Lambda
$$

(' indicates prime, and does not indicate transpose) where

$$
C_{\text {reduced }}^{\prime}=\left[\begin{array}{cccc}
1 & 1 & \cdots & 1 \\
e^{i\left(\vec{v}_{2}-\vec{v}_{1}\right) \bullet \vec{a}_{2}} & e^{i\left(\vec{v}_{2}-\vec{v}_{1}\right) \bullet \vec{a}_{3}} & \cdots & e^{i\left(\vec{v}_{2}-\vec{v}_{1}\right) \bullet \vec{a}_{N a n t}} \\
e^{i\left(\vec{v}_{3}-\vec{v}_{1}\right) \bullet \vec{a}_{2}} & e^{i\left(\vec{v}_{3}-\vec{v}_{1}\right) \bullet \vec{a}_{3}} & \cdots & e^{i\left(\vec{v}_{3}-\vec{v}_{1}\right) \bullet \vec{a}_{N_{a n t}}} \\
\vdots & \vdots & \ddots & \vdots \\
e^{i\left(\vec{v}_{N}-\vec{v}_{1}\right) \bullet \vec{a}_{2}} & e^{i\left(\vec{v}_{N}-\vec{v}_{1}\right) \bullet \vec{a}_{3}} & \cdots & e^{i\left(\vec{v}_{N}-\vec{v}_{1}\right) \bullet \vec{a}_{N a n t}}
\end{array}\right]
$$

and

$$
\Lambda=\left[\begin{array}{ccccc}
e^{i \vec{v}_{1} \bullet \vec{a}_{2}} & 0 & 0 & \cdots & 0 \\
0 & e^{i \vec{v}_{1} \bullet \vec{a}_{3}} & 0 & \cdots & 0 \\
0 & 0 & e^{i \vec{v}_{1} \bullet \vec{a}_{4}} & \cdots & 0 \\
\vdots & \vdots & \vdots & \ddots & \vdots \\
0 & 0 & 0 & \cdots & e^{i \vec{v}_{1} \bullet \vec{a}_{N a n t}}
\end{array}\right] .
$$


(Note that the vectors $\vec{v}$ have units of inverse meters, $\left(\mathrm{m}^{-1}\right)$ and the vectors $\vec{a}$ have unit of meters.) So

$$
C_{\text {reduced }}\left[\begin{array}{c}
A_{2} \\
A_{3} \\
\vdots \\
A_{N_{\text {ant }}}
\end{array}\right]=C_{\text {reduced }}^{\prime} \Lambda\left[\begin{array}{c}
A_{2} \\
A_{3} \\
\vdots \\
A_{N_{a n t}}
\end{array}\right]=\left[\begin{array}{c}
-1 \\
-1 \\
\vdots \\
-1
\end{array}\right]
$$

and now the criterion for existence of a solution is that $[-1-1 \cdots,-1]^{T}$ must lie in the column space of $C_{\text {reduced }}^{\prime}$.

Note that if $C_{\text {reduced }}^{\prime}$ takes the form

$$
C_{\text {reduced }}^{\prime}=\left[\begin{array}{cccc}
1 & 1 & \cdots & 1 \\
\vdots & \vdots & \cdots & \vdots \\
\gamma & \gamma & \cdots & \gamma \\
\vdots & \vdots & \cdots & \vdots
\end{array}\right]
$$

i.e., has a row of identical entries (besides the first row), with $\gamma \neq 1$, then the columns cannot span the vector $[-1-1-1 \cdots-1]^{T}$. So if a configuration of jammers is chosen such that all entries of, say, the second row of $C_{\text {reduced }}^{\prime}$ are equal but different from 1 , then there are no solutions. We explore this possibility.

The second row of $C_{\text {reduced }}^{\prime}$ will have equal entries if

$$
\left(\vec{v}_{2}-\vec{v}_{1}\right) \bullet \vec{a}_{2}=\left(\vec{v}_{2}-\vec{v}_{1}\right) \bullet \vec{a}_{3}+\alpha_{3} 2 \pi=\cdots\left(\vec{v}_{2}-\vec{v}_{1}\right) \bullet \vec{a}_{N_{a n t}}+\alpha_{N_{a n t}} 2 \pi,
$$

where $\alpha_{3}, \cdots, \alpha_{N_{a n t}}$ are integers. In order to prevent $\gamma=e^{i\left(\vec{v}_{2}-\vec{v}_{1}\right) \bullet \vec{a}_{2}}=1$ we require

$$
\left(\vec{v}_{2}-\vec{v}_{1}\right) \bullet \vec{a}_{2} \neq \alpha 2 \pi
$$

for any integer $\alpha$. Note that equations (14) and (15) are conditions only on the difference $\vec{v}_{2}-\vec{v}_{1}$, if there is one solution, there are infinitely many. 
Now, consider a specific example of finding confounding jammers for a given array. The array from figure 4 has $\vec{a}_{1}=0, \vec{a}_{2}=\hat{i}$, and $\vec{a}_{3}=\frac{1}{2} \hat{i}+\frac{\sqrt{3}}{2} \hat{j}$ and we will confound it with 2 jammers. Let $\vec{v}_{2}-\vec{v}_{1}=\kappa \hat{i}+\nu \hat{j}$. Then

$$
\begin{gathered}
\left(\vec{v}_{2}-\vec{v}_{1}\right) \bullet \vec{a}_{2}=\kappa \\
\left(\vec{v}_{2}-\vec{v}_{1}\right) \bullet \vec{a}_{3}=(\kappa \hat{i}+\nu \hat{j}) \bullet\left(\frac{1}{2} \hat{i}+\frac{\sqrt{3}}{2} \hat{j}\right)=\frac{\kappa}{2}+\frac{\sqrt{3} \nu}{2} .
\end{gathered}
$$

To satisfy equation (14), set

$$
\left(\vec{v}_{2}-\vec{v}_{1}\right) \bullet \vec{a}_{3}+\alpha_{3} 2 \pi=\frac{\kappa}{2}+\frac{\sqrt{3} \nu}{2}+\alpha_{3} 2 \pi=\left(\vec{v}_{2}-\vec{v}_{1}\right) \bullet \vec{a}_{2}=\kappa
$$

and solve for $\nu$

$$
\nu=\frac{1}{\sqrt{3}}\left(\kappa+4 \pi \alpha_{3}\right)
$$

Thus

$$
\vec{v}_{2}-\vec{v}_{1}=\kappa \hat{i}+\frac{1}{\sqrt{3}}\left(\kappa+4 \pi \alpha_{3}\right) \hat{j} .
$$

Pick $\alpha_{3}=1$ and $\kappa=1$, then from equation (16), $\nu=7.8325$ and

$$
\vec{v}_{2}-\vec{v}_{1}=\hat{i}+7.8325 \hat{j}
$$

Since the direction and amplitude of $\vec{v}_{1}$ is not determined, pick $\left|\vec{v}_{1}\right|=32.987 \mathrm{~m}^{-1}$ and choose the direction as $\vec{v}_{1}=16 \hat{i}+28.85 \hat{j}$. Then $\vec{v}_{2}$ is, from equation (17), $\vec{v}_{2}=-15 \hat{i}-21.0175 \hat{j}$ and its magnitude is $\left|\vec{v}_{2}\right|=25.82 \mathrm{~m}^{-1}$.

The wavelength of jammer \#1 is

$$
\lambda_{1}=2 \pi /\left|\vec{v}_{1}\right|=0.19047 \mathrm{~m}
$$

and the wavelength of jammer \#2 is

$$
\lambda_{2}=2 \pi /\left|\vec{v}_{2}\right|=2 \pi /\left|\vec{v}_{1}+\kappa \hat{i}+\frac{1}{\sqrt{3}}\left(\kappa+4 \pi \alpha_{3}\right) \hat{j}\right|=0.243 \mathrm{~m}
$$


The frequencies corresponding to the wavelengths of these 2 jammers that will confound the 3 element equilateral triangle antenna array are $f_{1}=1.575 \mathrm{GHz}$ and $f_{2}=1.23 \mathrm{GHz}$, which are close to the wavelengths of the GPS transmissions.

Another example of an array that can be confounded with 2 jammers is the centered square array. The antenna location vectors are $\vec{a}_{1}=0, \vec{a}_{2}=\hat{i}, \vec{a}_{3}=\hat{j}, \vec{a}_{4}=-\hat{i}$, and $\vec{a}_{5}=-\hat{j}$. Let $\vec{v}_{2}-\vec{v}_{1}=\kappa \hat{i}+\nu \hat{j}$. Notice that $\left(\vec{v}_{2}-\vec{v}_{1}\right) \bullet \vec{a}_{2}=\kappa$ and $\left(\vec{v}_{2}-\vec{v}_{1}\right) \bullet \vec{a}_{4}=-\kappa$ so equation (14) requires

$$
\kappa=-\kappa+\alpha_{4} 2 \pi=\alpha_{4} \pi
$$

If $\alpha_{4}$ is an even integer, this contradicts the requirement (equation (15)) that $\left(\vec{v}_{2}-\vec{v}_{1}\right) \bullet \vec{a}_{2}$ cannot be an integer multiple of $2 \pi$; so choose $\kappa$ to be an odd multiple of $\pi$ :

$$
\kappa=\left(2 N_{1}+1\right) \pi
$$

where $N_{1}$ is any integer. Equation (14) also requires

$$
\left(\vec{v}_{2}-\vec{v}_{1}\right) \bullet \vec{a}_{3}=\nu \quad \text { and } \quad\left(\vec{v}_{2}-\vec{v}_{1}\right) \bullet \vec{a}_{5}=-\nu
$$

so

$$
\begin{aligned}
\nu+\alpha_{3} 2 \pi & =-\nu+\alpha_{5} 2 \pi \\
\nu & =\left(\alpha_{5}-\alpha_{3}\right) \pi \\
& \equiv\left(2 N_{2}+1\right) \pi
\end{aligned}
$$

where $N_{2}$ is any integer. The centered square array can be confounded by any 2 jammers related by $\vec{v}_{2}-\vec{v}_{1}=\left(2 N_{1}+1\right) \pi \hat{i}+\left(2 N_{2}+1\right) \pi \hat{j}$.

Arbitrarily choosing $N_{1}=1$ and $N_{2}=2$ results in $\kappa=3 \pi$ and $\nu=5 \pi$. Again, the direction and amplitude of $\vec{v}_{1}$ can be anything. Choosing $\left|\vec{v}_{1}\right|=32.987 \mathrm{~m}^{-1}$ and its direction as $\vec{v}_{1}=20 \hat{i}+26.23 \hat{j}$ results in $\vec{v}_{2}=29.42 \hat{i}+41.94 \hat{j}$ and $\left|\vec{v}_{2}\right|=51.23 \mathrm{~m}^{-1}$. 
The wavelengths of the jammers are

$$
\lambda_{1}=2 \pi /\left|\vec{v}_{1}\right|=0.1905 \mathrm{~m}
$$

and

$$
\lambda_{2}=2 \pi /\left|\vec{v}_{1}+\left(2 N_{1}+1\right) \pi \hat{i}+\left(2 N_{2}+1\right) \pi \hat{j}\right|=0.1226 \mathrm{~m} .
$$

The frequencies of these jammers are $f_{1}=1.575 \mathrm{GHz}$ and $f_{2}=2.45 \mathrm{GHz}$. Note that we have confounded a five antenna array with only two jammers.

This particular method of finding jammers to confound an array will not work if all the antenna location vectors are related by $\vec{a}_{i}-\vec{a}_{j}=\vec{a}_{k}$ for some $i, j, k$. An example of such an array is a hexagonal array with $\vec{a}_{1}=0, \vec{a}_{2}=\hat{i}, \vec{a}_{3}=\frac{1}{2} \hat{i}+\frac{\sqrt{3}}{2} \hat{j}, \vec{a}_{4}=-\frac{1}{2} \hat{i}+\frac{\sqrt{3}}{2} \hat{j}, \vec{a}_{5}=-\hat{i}, \vec{a}_{6}=$ $-\frac{1}{2} \hat{i}-\frac{\sqrt{3}}{2} \hat{j}$, and $\vec{a}_{7}=\frac{1}{2} \hat{i}-\frac{\sqrt{3}}{2} \hat{j}$. For this array, $\vec{a}_{3}-\vec{a}_{4}=\vec{a}_{2}, \vec{a}_{4}-\vec{a}_{3}=\vec{a}_{5}, \vec{a}_{3}-\vec{a}_{2}=\vec{a}_{4}$, etc. Substituting one of these, for example $\vec{a}_{2}=\vec{a}_{3}-\vec{a}_{4}$, into equation (14) and rearranging terms results in

$-\left(\vec{v}_{2}-\vec{v}_{1}\right) \bullet \vec{a}_{4}=\alpha_{3} 2 \pi=\left(\vec{v}_{2}-\vec{v}_{1}\right) \bullet\left(\vec{a}_{4}-\vec{a}_{3}\right)+\alpha_{4} 2 \pi=\cdots=\left(\vec{v}_{2}-\vec{v}_{1}\right) \bullet\left(\vec{a}_{N_{a n t}}-\vec{a}_{3}\right)+\alpha_{N_{a n t}} 2 \pi$

but this leaves the term $\left(\vec{v}_{2}-\vec{v}_{1}\right) \bullet \vec{a}_{4}$ equal to an integer multiple of $2 \pi$. This violates the condition of equation (15) defeating the objective of finding a confounding configuration.

Summarizing, note that the HSB gradient algorithm as well as other algorithms focus on minimizing the total power output from the array, $\left|\left[\chi_{G P S}+N+J C\right] A\right|^{2}$, while the oracle focuses on zeroing the term $C A$. So if the oracle solution exists and $\chi_{G P S}$ and $N$ are small, it is a reasonable standard for assessing the HSB gradient algorithm; if the oracle solution does not exist then the goal of the HSB gradient algorithm may be unachievable.

The next chapter describes the HSB gradient adaptive algorithm that computes antenna weights which seek to minimize the output power of an array. A comparison between the HSB gradient weights and the oracle weights is made by comparing, at each step of the simulation, the array output powers resulting from each set of weights. The comparison 
shows that the HSB algorithm exhibits excellent performance in minimizing the output power of an array. 


\section{Chapter 4}

\section{Hilbert-Space-Based (HSB) Gradient Algorithm}

This chapter will describe the theory of the HSB gradient algorithm. The objective of this algorithm is to minimize the output power of the array. The signals of interest received from the GPS satellite network are spread spectrum signals of negligible power, about the same as the background noise level. Reducing the array output power to this level will allow GPS signals to be extracted.

\subsection{Power Minimization and Weight Calculation}

At time $t_{\gamma}$, the output of antenna \#m consisting of GPS signals, noise, and $N_{\text {jam }}$ jammers is

$$
A_{m} \chi_{G P S}^{(m)}\left(t_{\gamma}\right)+A_{m} N_{m}\left(t_{\gamma}\right)+\sum_{n=1}^{N_{\text {jam }}} J_{n} e^{i \omega_{n} t_{\gamma}} e^{i \frac{2 \pi}{\lambda_{n}} \vec{a}_{m} \bullet \vec{j}_{n}} A_{m}
$$

where $N_{m}\left(t_{\gamma}\right)$ conceptually represents a random noise component in antenna \#m. The details of the nature of the random noise in the array are discussed in section 4.3.

The array output is the sum of all antenna outputs

$$
\sum_{m=1}^{N_{a n t}} A_{m} \chi_{G P S}^{(m)}\left(t_{\gamma}\right)+\sum_{m=1}^{N_{\text {ant }}} A_{m} N_{m}\left(t_{\gamma}\right)+\sum_{m=1}^{N_{\text {ant }}} \sum_{n=1}^{N_{\text {jam }}} J_{n} e^{i \omega_{n} t_{\gamma}} e^{i \frac{2 \pi}{\lambda_{n}} \vec{a}_{m} \bullet \vec{j}_{n}} A_{m}
$$

which can be expressed in matrix form, from (5), as

$$
\chi_{G P S} A+N A+J C A=x A .
$$

The power minimization and weight calculations will proceed as follows. For some initial choice of weights $A$, the output power of the array is sampled every $\tau$ seconds and assessed at times labeled by the variable $t_{\gamma}=\gamma \tau$ ( $\gamma$ is an integer $), \Gamma$ of these power measurements 
are averaged every $\Gamma \tau$ seconds, and the gradient of this average (with respect to the weights) is calculated. The peripheral antenna weights are adjusted in the direction of the negative gradient. This constitutes one algorithm iteration. It is unlikely that one single adjustment in each weight value results in their optimal values so these updated weights are used to accumulate another batch of $\Gamma$ output power measurements, and the algorithm is repeated. If the procedure converges, the search becomes more precise, with finer adjustments in the weight values toward their optimal values, as the algorithm progresses.

The instantaneous output power of the array, $\mathrm{p}\left(t_{\gamma}, A\right)$, is

$$
\mathrm{p}\left(t_{\gamma}, A\right)=(x A)(x A)^{\dagger}
$$

where $^{\dagger}$ represents conjugate transpose. For each value of $t_{\gamma}, \mathrm{p}\left(t_{\gamma}, A\right)$ represents one sample of the instantaneous array output power.

During the $k$ th iteration of the algorithm, $\Gamma$ samples of instantaneous power, $\mathrm{p}\left(t_{\gamma}, A\right)$, are averaged to obtain the average output power of the array, $P(k, A)$, i.e.,

$$
P(k, A)=\frac{1}{\Gamma} \sum_{t_{\gamma}=(k-1) \Gamma+1}^{k \Gamma} \mathrm{p}\left(t_{\gamma}, A\right) .
$$

The average output power is a quadratic function of the antenna weights. To help picture this power function, consider an array of 3 antennas, all with real weights only, in any configuration (2 peripheral antennas with adjustable weights and 1 reference antenna with a fixed weight). The output power of the array can be plotted along the $z$-axis as a function of the adjustable antenna weights represented along the $x$ and $y$ axes. The power surface is shaped like a bowl with a single, unique minimum. The antenna weights that correspond to this minimum are those that the algorithm seeks. The strategy is to converge to the minimum by continuously adjusting the values for the antenna weights so as to enforce $P(k+1, A)<P(k, A)$.

Recall, from Chapter $2, A$ is a $1 \times N_{\text {ant }}$ vector with components $w_{m} e^{i q_{m}}, m=1,2, \ldots, N_{\text {ant }}$. The power $P(k, A)$ can be expressed explicitly in terms of the magnitudes, $w_{m}$, and phases, 
$q_{m}$, of the antenna weights by reincarnating $A_{m}=w_{m} e^{i q_{m}}$ as a new variable $W$, where $W$ is a column of antenna weight parameters $\left[\begin{array}{llll}w_{1} & w_{2} & \cdots & w_{N_{a n t}} \\ q_{1} & q_{2} \cdots q_{N_{a n t}}\end{array}\right]^{T}$ (recall since $A_{1} \equiv 1, w_{1}=1$ and $\left.q_{1}=0\right)$. The objective of the algorithm is to choose the change in the weights, $\Delta W_{m}$, to reduce $P\left(k, W_{m}\right)$ :

$$
P\left(k+1, W_{m}+\Delta W_{m}\right) \leq P\left(k, W_{m}\right), \quad m=1,2, \ldots, 2 N_{\text {ant }} .
$$

Without risk of confusion, we write $P(W)$ for $P(k, A)$. Expanding $P\left(W_{m}+\Delta W_{m}\right)$ with a (matrix) Taylor series to first order yields

$$
P\left(W_{m}+\Delta W_{m}\right) \approx P\left(W_{m}\right)+\Delta W^{T} \frac{\partial P}{\partial W} \equiv P\left(W_{m}\right)+\Delta W^{T} \nabla P \leq P\left(W_{m}\right),
$$

where $W$ is the column of weight parameters introduced above and $\nabla P$ represents the column of all components of the gradient of the output power with respect to the antenna weight parameters, $\nabla P=\left[\frac{\partial P}{\partial w_{1}}, \frac{\partial P}{\partial w_{2}}, \ldots, \frac{\partial P}{\partial w_{N_{a n t}}}, \frac{\partial P}{\partial q_{1}}, \frac{\partial P}{\partial q_{2}}, \ldots, \frac{\partial P}{\partial q_{N_{a n t}}}\right]^{T}$.

For the steepest descent algorithm, the weights from iteration $k$ to iteration $k+1$ are adjusted in the direction of the negative power gradient with a scale factor $\mu$, the "step size"

$$
\Delta W=-\mu \nabla P
$$

Substituting equation (24) for $\Delta W^{T}$ in equation (23) results in

$$
\Delta P=P(W+\Delta W)-P(W) \approx-\mu \nabla P^{T} \nabla P .
$$

The desired change in power is known; it is the difference between the present power, $P(k, A)=P(W)$ and the minimum power, which is approximately zero.

$$
\Delta P=\text { change in power }=(0-P(W))=-\mu \nabla P^{T} \nabla P
$$


and solving for the step size $\mu$, one obtains

$$
\mu=\frac{P(W)}{\nabla P^{T} \nabla P}
$$

The weight adjustment equation (24) becomes

$$
\Delta W=-\mu \nabla P=\frac{-P(W)}{\nabla P^{T} \nabla P} \nabla P
$$

Equation (27) in terms of the weight magnitudes states

$$
\Delta w_{i}=-\mu \frac{\partial P}{\partial w_{i}}=-P(W) \times\left(\frac{\partial P / \partial w_{i}}{\sum_{m=2}^{N_{a n t}}\left[\left(\frac{\partial P}{\partial w_{m}}\right)^{2}+\left(\frac{\partial P}{\partial q_{m}}\right)^{2}\right]}\right), i=2,3, \ldots, N_{\text {ant }}
$$

and in terms of the weight phases it states

$$
\Delta q_{i}=-\mu \frac{\partial P}{\partial q_{i}}=-P(W) \times\left(\frac{\partial P / \partial q_{i}}{\sum_{m=2}^{N_{\text {ant }}}\left[\left(\frac{\partial P}{\partial w_{m}}\right)^{2}+\left(\frac{\partial P}{\partial q_{m}}\right)^{2}\right]}\right), i=2,3, \ldots, N_{\text {ant }} .
$$

These weight change calculations occur once per algorithm iteration, each time with updated values for $\mu$ and $\nabla P$. Our innovation for calculating the gradient components, $\frac{\partial P}{\partial w_{m}}$ and $\frac{\partial P}{\partial q_{m}}$, will be described in the next section.

If the array is in motion, the optimal weight search performed by the algorithm becomes nonstationary because the power surface changes at each iteration. The power surface is a function of the phase matrix $C$ which is a function of the changing jammer arrival directions, $\vec{j}_{n}$. For an array in motion, the power is time averaged and each weight is updated per algorithm iteration as it is for a stationary array. 


\subsection{Exact Gradient Calculation}

This section will describe the computation of the gradient of the average array output power. What distinguishes the HSB gradient algorithm from all other gradient based adaptive algorithms is that it calculates the exact gradient components, not approximations of the gradient components. Furthermore, the algorithm calculates the gradient components simultaneously and efficiently.

The power gradient, $\nabla P$, is computed using the Hilbert space inner products of signals which are readily available at the array output by the following method [5]. From equations (21) and (22),

$$
P(k, A)=\frac{1}{\Gamma} \sum_{t_{\gamma}=(k-1) \Gamma+1}^{k \Gamma} \mathrm{p}\left(t_{\gamma}, A\right)=\frac{1}{\Gamma} \sum_{t_{\gamma}=(k-1) \Gamma+1}^{k \Gamma}(x A)(x A)^{\dagger}
$$

Rewrite this summation using inner product bra/ket notation

$$
<a, b>=\frac{1}{T} \sum_{t=(k-1) T+1}^{k T} a(t) b^{\dagger}(t)
$$

and the power is represented as

$$
P(k, A)=<x A, x A>
$$

Since the array output results from the $N_{\text {ant }}$ antenna outputs,

$$
x A=\sum_{m=1}^{N_{\text {ant }}} A_{m} x_{m}(k)
$$

the array output power becomes

$$
P(k, A)=<\sum_{g=1}^{N_{a n t}} A_{g} x_{g}(k), \sum_{g=1}^{N_{a n t}} A_{g} x_{g}(k)>=\sum_{g=1}^{N_{a n t}} \sum_{h=1}^{N_{a n t}} A_{g} A_{h}^{*}<x_{g}(k), x_{h}(k)>
$$


Now the $m$ th component of the gradient with respect to the weight magnitude $w_{m}$ is (the time dependence of $x$ is dropped for clarity) (recall $A_{g}=w_{g} e^{i q_{g}}$ )

$$
\begin{aligned}
\frac{\partial P}{\partial w_{m}} & =\frac{\partial\left(\sum_{g=1}^{N_{a n t}} \sum_{h=1}^{N_{a n t}} A_{g} A_{h}^{*}<x_{g}, x_{h}>\right)}{\partial w_{m}} \\
& =\sum_{g} \sum_{h}<x_{g}, x_{h}>A_{g} \frac{\partial w_{h} e^{-i q_{h}}}{\partial w_{m}}+\sum_{g} \sum_{h}<x_{g}, x_{h}>\frac{\partial w_{g} e^{i q_{g}}}{\partial w_{m}} A_{h}^{*}
\end{aligned}
$$

On the right hand side of this equation, the partial derivative with respect to $w_{m}$ is non-zero only when $h=m$ for the first term and only when $g=m$ for the second term:

$$
\begin{aligned}
\frac{\partial P}{\partial w_{m}} & =\sum_{g} \sum_{h}<x_{g}, x_{h}>A_{g} \delta_{h m} e^{-i q_{m}}+\sum_{g} \sum_{h}<x_{g}, x_{h}>\delta_{g m} e^{i q_{m}} A_{h}^{*} \\
& =\sum_{g}<x_{g}, x_{m}>A_{g} e^{-i q_{m}}+\sum_{h}<x_{m}, x_{h}>e^{i q_{m}} A_{h}^{*} .
\end{aligned}
$$

Rearranging terms and identifying the summation of the antenna outputs as the array output signal, $x A$ from equation (31), yields

$$
\begin{aligned}
\frac{\partial P}{\partial w_{m}} & =\sum_{g}<A_{g} x_{g}, e^{i q_{m}} x_{m}>+\sum_{h}<e^{i q_{m}} x_{m}, A_{h} x_{h}> \\
& =<x A, A_{m} x_{m}>/ w_{m}+<A_{m} x_{m}, x A>/ w_{m} .
\end{aligned}
$$

The terms in the last line are complex conjugates of each other. A quantity added to its conjugate leaves only twice the real part

$$
\frac{\partial P}{\partial w_{m}}=\frac{2 \Re<A_{m} x_{m}, x A>}{w_{m}} .
$$

The components of the gradient with respect to the weight magnitudes are simply the Hilbert space inner products of the real parts of the appropriately scaled (by $\frac{1}{w_{m}}$ ) individual output signals of each antenna $A_{m} x_{m}(k)$ with the array signal output, $x(k) A$. That is, they are correlations (at zero delay). 
Similarly, the $m$ th component of the gradient with respect to the phase $q_{m}$ of the weight is

$$
\begin{aligned}
\frac{\partial P}{\partial q_{m}} & =\frac{\partial\left(\sum_{g}^{N_{a n t}} \sum_{h}^{N_{a n t}} A_{g} A_{h}^{*}<x_{g}, x_{h}>\right)}{\partial q_{m}} \\
& =\sum_{g} \sum_{h}<x_{g}, x_{h}>A_{g} \frac{\partial w_{h} e^{-i q_{h}}}{\partial q_{m}}+\sum_{g} \sum_{h}<x_{g}, x_{h}>\frac{\partial w_{g} e^{i q_{g}}}{\partial q_{m}} A_{h}^{*}
\end{aligned}
$$

On the right hand side of this equation, the partial derivative with respect to $q_{m}$ is non-zero only when $h=m$ for the first term and only when $g=m$ for the second term:

$$
\begin{aligned}
\frac{\partial P}{\partial q_{m}}= & \sum_{g} \sum_{h}<x_{g}, x_{h}>A_{g} \delta_{h m} w_{h}(-i) e^{-i q_{h}} \\
& +\sum_{g} \sum_{h}<x_{g}, x_{h}>\delta_{g m} w_{g}(i) e^{i q_{g}} A_{h}^{*} \\
= & -i \sum_{g}<x_{g}, x_{m}>A_{g} A_{m}^{*}+i \sum_{h}<x_{m}, x_{h}>A_{m} A_{h}^{*}
\end{aligned}
$$

Rearranging terms and identifying the summation of the antenna outputs as the array output signal, $x A$ from equation (31), yields

$$
\begin{aligned}
\frac{\partial P}{\partial q_{m}} & =-i \sum_{g}<A_{g} x_{g}, A_{m} x_{m}>+i \sum_{h}<A_{m} x_{m}, A_{h} x_{h}> \\
& =-i<x A, A_{m} x_{m}>+i<A_{m} x_{m}, x A> \\
& =2 \Im<A_{m} x_{m}, x A>.
\end{aligned}
$$

The components of the gradient with respect to the weight phases are simply the Hilbert space inner products of the imaginary parts of the scaled individual output signals of each antenna $A_{m} x_{m}(k)$ with the array signal output, $x(k) A$.

With all components of the gradient available, they are then used to determine the antenna weights to minimize the output power from the array as in equations (28) and (29).

To reiterate, the components of the gradient are found using the equations

$$
\frac{\partial P}{\partial w_{m}}=\frac{2 \Re<A_{m} x_{m}, x A>}{w_{m}}
$$


and

$$
\frac{\partial P}{\partial q_{m}}=2 \Im<A_{m} x_{m}, x A>
$$

These components, again, are not approximations of the gradient, they are the exact components of the gradient. The estimates of the gradient used by other algorithms only approximate the gradient. Algorithms that use finite differences to approximate the gradient lose accuracy, particularly near the minimum of the quadratic surface, and take multiple time steps to evaluate, since each term in the finite difference requires a separate power average. The simultaneous, efficient, exact calculation of all components of the gradient by the method proposed herein leads to faster, more robust jammer cancellation performance.

Although we do not exploit it in this study, it is of interest to note that these inner products can be evaluated using power averages alone, for judiciously chosen signal combinations

$$
\frac{\partial P}{\partial w_{m}}=\frac{1}{w_{m}}\left\{<A_{m} x_{m}+x A, A_{m} x_{m}+x A>-<A_{m} x_{m}, A_{m} x_{m}>-<x A, x A>\right\}
$$

and

$$
\frac{\partial P}{\partial q_{m}}=-i\left\{<A_{m} x_{m}+i x A, A_{m} x_{m}+i x A>-<A_{m} x_{m}, A_{m} x_{m}>-<x A, x A>\right\} .
$$

\subsection{Noise Simulation}

Up to now this chapter has focused on the implementation of the algorithm; this section will describe details about noise in the simulation of the algorithm. Noise is added as random 'input' to the array; it is subject to scaling by the antenna weights as are the jammer and GPS input signals.

The objective of the HSB gradient algorithm is to minimize the output power of the array. During each iteration, noise is simulated as random samples and input to each antenna. These samples are added to the jammer and GPS input signals and these composite signals are scaled by the antenna weights and combined to form the array output. 
Referring to equation (20), the sample at time $t_{\gamma}$ of the array output signal is modeled as

$$
\chi_{G P S} A+N A+J C A=x A .
$$

The noise term in this equation is

$$
N A=\sum_{m=1}^{N_{a n t}} N_{m}\left(t_{\gamma}\right) A_{m}
$$

where at each time step $N_{m}\left(t_{\gamma}\right)$ is modeled as an independent sample of a random variable with a Gaussian distribution of zero mean and variance $\sigma^{2}$. The power in this noise at the output of the array must equal the (specified) background noise level, $\rho$. It is known that the sum of $N_{\text {ant }}$ random variables, each with the same mean, $\mu$, and variance, $\sigma^{2}$, has mean equal to $N_{\text {ant }} \mu$ and variance equal to $N_{\text {ant }} \sigma^{2}$ [8]. Setting the standard deviation of each sample of noise at each antenna equal to $\sqrt{\rho / N_{\text {ant }}}$ fixes the power level of the noise at the output of the array to match that of the background noise.

\subsection{Differences in Algorithm and Oracle Solution}

A direct comparison of the antenna weights of the HSB gradient algorithm with another algorithm is not possible because the antenna weights approach their optimal values via different paths, depending on the details of the adaptive algorithm. However, as discussed in Chapter 3, the results of any iteration of the antenna weights of the HSB gradient algorithm can be compared with the oracle weights for the corresponding antenna position. Some difference between the algorithm and oracle weights is expected because the oracle provides the exact weights annihilating only the output power component due to jammers, $|J C A|^{2}$, while the algorithm weights minimize the output power of the array due to all inputs, $\left|\chi_{G P S} A+N A+J C A\right|^{2}$.

The HSB gradient algorithm and the oracle also differ in their dependence on the jammer amplitudes. The HSB gradient algorithm uses the jammer amplitude information in finding the solution for the antenna weights. The jammer amplitudes and arrival directions affect 
the output signal of the array, the output power of the array, the gradient, and the weight update equation. Every antenna in the array is affected by every jammer arriving at the array. A weak jammer cannot remove or reduce the effect that a particular antenna weight has in the array nor can a strong jammer cause a particular antenna weight to dominate the algorithm solution. All jammers affect all antenna weights. What determines the algorithm's solution for effective power minimizing antenna weights is how close the search of the power surface in the negative gradient direction approaches the minimum.

The oracle solution does not take into account any information about the amplitudes of the jammers. The oracle solves $C A=0$ for the optimal weights to annihilate the array output. The jammer amplitudes, $J$, do not affect the values of the oracle antenna weights. The arrival directions of the jammers, however, are critical to the solution. They are used in the elements of $C$. 


\section{Chapter 5}

\section{Matlab Implementation}

This chapter will describe the implementation of the HSB gradient algorithm in Matlab. Since the theory of the algorithm has already been presented in the previous chapter, this chapter is included only for readers who are interested in coding details. The results of the simulations are reported in Chapter 6.

The input to the array is modeled as high power jammers and low power noise. The GPS signals are not modeled explicitly, although they would appear similar to the noise. The algorithm does not perform any demodulation of the GPS signals. The HSB gradient algorithm is implemented in Matlab with 3 files: setup.m, adapt.m and motion.m.

\section{$5.1 \quad$ setup.m}

The file setup.m defines and initializes constants necessary for the adaptive algorithm implementation. See Table 1 for a list of these constants.

Setup.m also defines variables that are initialized by the user. Table 2 lists these variables along with typical values they might be assigned.

The peripheral antennas in the array can be arranged in any configuration around a central antenna. Here the antennas are arranged in a plane with the origin of the reference coordinate system coinciding with the central antenna. The peripheral antenna locations are measured counterclockwise from the $x$-axis at angles $\gamma_{m}, m=2,3, \ldots, N_{\text {ant }}$. Each jammer arrives at the array with an azimuth angle $\phi$ measured counterclockwise from the $x$-axis and with an elevation angle $\theta$ measured down from the $z$-axis. 
Table 1. Constants in setup.m.

\begin{tabular}{|l|l|l|}
\hline Variable Name & Value & Description \\
\hline deg_to_rad & $\pi / 180$ & degrees to radians conversion constant \\
\hline jmath & $\sqrt{-1}$ & imaginary number definition \\
\hline A & $\begin{array}{l}2 \times \text { Nant matrix, all } \\
\text { row } 1 \text { entries }=1, \text { all } \\
\text { row } 2 \text { entries }=0\end{array}$ & $\begin{array}{l}\text { initial antenna weight magnitudes and } \\
\text { phases }\end{array}$ \\
\hline a & $1 \times$ Nant & complex weight values \\
\hline Ann & $2 \times$ Nant & $\begin{array}{l}\text { initial antenna weight magnitudes and } \\
\text { phases for no noise case }\end{array}$ \\
\hline ann & $1 \times$ Nant & complex weight values for no noise case \\
\hline speed_of_light & $3 \times 10^{8} \mathrm{~m} / \mathrm{s}$ & speed of light constant \\
\hline P_int & $20 \times 10^{-6}$ sec & power integral duration \\
\hline LOfreq & $1.575 x 10^{9} \mathrm{~Hz}$ & local oscillator frequency \\
\hline omegaLO & $2 \pi$ LOfreq rad/sec & local oscillator frequency \\
\hline jam_freq & L0freq & frequency of jammers \\
\hline omega_jam & $2 \pi j$ am_freq & frequency of jammers \\
\hline motion_vector & {$[$ roll,pitch,yaw $]$} & $\begin{array}{l}\text { vector of variables indicating array mo- } \\
\text { tion }\end{array}$ \\
\hline az, el, rr & & $\begin{array}{l}\text { spherical coordinates of jammer arrival } \\
\text { angles }\end{array}$ \\
\hline phi_jam & $\begin{array}{l}\text { azimuth jammer arrival angle, counter- } \\
\text { clockwise from } x \text {-axis }\end{array}$ \\
\hline theta_jam & $\begin{array}{l}\text { az } \\
\text { from } z \text {-axis }\end{array}$ \\
\hline gamma & $\begin{array}{l}\text { vector of angles describing antenna lo- } \\
\text { cations, counterclockwise from } x \text {-axis }\end{array}$ \\
\hline
\end{tabular}

The sampling rate of the jammer and noise signals is set to $20 \mathrm{MHz}(\tau=50 \mathrm{~ns})$. Every iteration of the algorithm processes $\Gamma=400$ samples or $20 \mu$ s of data. These 400 samples of instantaneous array output are averaged to form one sample of the average array output power per iteration. The variable $\mathrm{P}_{-}$int represents the duration of one iteration.

The phase matrix, $C$ is calculated in setup.m. The matrix is made up of the exponential phase differences between the jammer wavefronts impinging on each peripheral antenna with respect to the center antenna in the array. $C$ has size $\mathrm{Njams} \times \mathrm{Nant}$ (from Chapter $3, N_{\text {jam }}=\mathrm{Nj}$ ams and $\left.N_{\text {ant }}=\mathrm{Nant}\right)$ and the $(n, m)$ th element has the form using the jammer arrival directions of $\phi$ and $\theta$ and antenna locations of $\gamma$

$$
C_{n m}=e^{i \frac{2 \pi}{\lambda} \vec{a}_{m} \bullet \vec{j}_{n}}=e^{i \frac{2 \pi}{\lambda} \cos \left(\gamma_{m}-\phi_{n}\right) \sin \theta_{n}}
$$


Table 2. Variables in setup.m Initialized by User.

\begin{tabular}{|l|l|l|}
\hline Variable Name & Typical Value & Description \\
\hline Nant & 7 & number of antennas in array \\
\hline array_radius & 0.1 & radius of array in meters \\
\hline a_min & 0.1 & minimum antenna weight magnitude \\
\hline a_max & 10 & maximum antenna weight magnitude \\
\hline step_control & 1 & $\begin{array}{l}\text { fraction of gradient correction to be im- } \\
\text { plemented }\end{array}$ \\
\hline noise_floor & $1.25(1=0 \mathrm{~dB})$ & target output power value \\
\hline Njams & 6 & number of jammers incident on array \\
\hline theta_jam & {$[90,90,90,90,90,90,-$} & $\begin{array}{l}\text { vector of jammer elevation angles, re- } \\
\text { duced to } 1 \times \mathrm{Nj} \text { ams }\end{array}$ \\
\hline phi_jam & $90,90,90]$ & $\begin{array}{l}\text { vector of jammer azimuth angles, re- } \\
\text { duced to } 1 \times \mathrm{Nj} \text { ams }\end{array}$ \\
\hline J & {$[30,90,150,210,270,-$} \\
& {$[100,100,100,100,-$} & $\begin{array}{l}\text { vector of jammer amplitudes, reduced } \\
\text { to } 1 \times \mathrm{Nj} \text { ams }\end{array}$ \\
\hline sim_time & $100,100,100,100,100]$ & $\begin{array}{l}\text { length of simulation, each iteration of } \\
\text { algorithm is } \mathrm{P} \text {-int long }\end{array}$ \\
\hline roll & $0.5 \mathrm{sec}$ & roll rate in deg/sec, $+y$ onto $+z$ \\
\hline pitch & 0 & pitch rate in deg/sec, $+z$ onto $-x$ \\
\hline yaw & 0 & yaw rate in deg/sec, $+y$ onto $+x$ \\
\hline beta & 360 & initial offset of array in degrees, - roll \\
\hline
\end{tabular}

where, in general, for a planar array

$$
\begin{gathered}
\vec{a}_{m}=\cos \gamma_{m} \hat{i}+\sin \gamma_{m} \hat{j}, \\
\vec{j}_{n}=\cos \phi_{n} \sin \theta_{n} \hat{i}+\sin \phi_{n} \sin \theta_{n} \hat{j}+\cos \theta_{n} \hat{k}
\end{gathered}
$$

and

$$
\vec{a}_{m} \bullet \vec{j}_{n}=\cos \left(\gamma_{m}-\phi_{n}\right) \sin \theta_{n}
$$

\section{2 adapt.m}

The file adapt.m performs the power, gradient, and weight adjustment calculations. The algorithm adapts the antenna weights for the duration sim_time, a length of time entered by the user. Since each iteration of the algorithm covers $\mathrm{P}$ int $=20 \mu \mathrm{s}$, the number of weight adjustment iterations performed is sim_time/P_int. At the start of an iteration, 


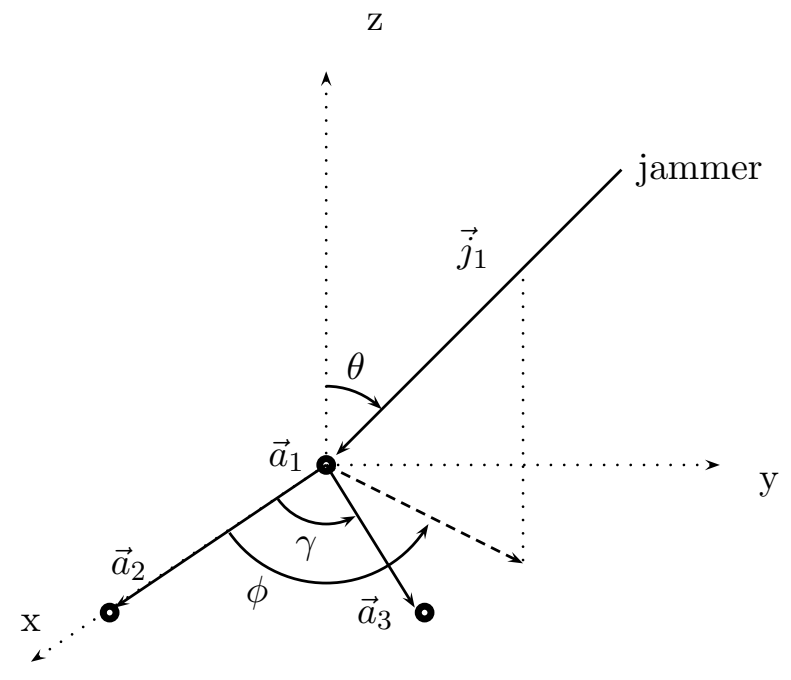

Figure 5. Example of $\vec{a}_{m}$ and $\vec{u}_{n}$.

motion_vector is checked for a nonzero value. If it is nonzero, the array has roll, pitch, and/or yaw motion and the file motion.m is called. This file updates the phase matrix $C$ due to the array motion. Details of motion.m are described in the next section.

With the phase matrix components calculated either from setup.m or from motion.m, the oracle weights are computed as in equation (10)

$$
\left[\begin{array}{c}
A_{2} \\
A_{3} \\
\vdots \\
A_{N_{\text {ant }}}
\end{array}\right]_{\text {or }}=-C_{\text {reduced }}^{-1}\left[\begin{array}{c}
1 \\
1 \\
\vdots \\
1
\end{array}\right]
$$

or $(11)$

$$
\left[\begin{array}{c}
A_{2} \\
A_{3} \\
\vdots \\
A_{N_{\text {ant }}}
\end{array}\right]_{\text {or }}=-C_{\text {reduced }}^{-\psi}\left[\begin{array}{c}
1 \\
1 \\
\vdots \\
1
\end{array}\right]
$$


where the subscript or indicates oracle weights. With the oracle weights known, the output power corresponding to the oracle weights is calculated using equation (21),

$$
\mathrm{p}_{\text {oracle }}\left(t_{\gamma}, A_{\text {or }}\right)=\left(x A_{\text {or }}\right)\left(x A_{\text {or }}\right)^{\dagger} \text {. }
$$

The condition number of $C_{\text {reduced }}$ is calculated per iteration using the cond function in Matlab.

To calculate the gradient of the power surface, the array output signal and array output power must be calculated first. As stated in section 4.3, the array output signal may contain noise as well as jammers. The simulated output signal of the array is

$$
N\left(t_{\gamma}\right) A+J\left(t_{\gamma}\right) C\left(t_{\gamma}\right) A=x\left(t_{\gamma}\right) A
$$

where the initial values of antenna weights, $A$, declared in setup.m, are used for the first iteration.

During each iteration, 400 samples of array output are simulated, $t_{\gamma}=\tau, 2 \tau, \ldots, 400 \tau$. For each value of $t_{\gamma}$, the noise, $N\left(t_{\gamma}\right)$, is a $1 \times N_{\text {ant }}$ vector of white noise samples simulated with the randn Matlab function. The Matlab randn function generates zero mean, unit variance white noise samples. The variance of the noise must be modified as described in section 4.3 to simulate a certain background noise level in the output signal of the array. This is accomplished by multiplying each noise sample at each antenna by the scalar $\sqrt{\frac{\text { noise floor }}{\text { Nant }}}$. Combining all Nant antenna outputs results in a noise signal at the output of the array with variance equal to noisefloor. The 400 noise samples are added to as many jammer components to form the input to each antenna. These signals are scaled by the antenna weights then summed together to form the array output.

Note that the phase matrix $C$ and the antenna weight vector $A$ are each modified only once per iteration. A snapshot of the array and jammer arrival directions is taken at the start of every algorithm iteration and held constant for the duration of one iteration. Recall that the objective of the algorithm is to minimize the output power of the array to within $5 \mathrm{~dB}$ of the background noise level. Each of the 400 samples of instantaneous output power 
per iteration is computed as

$$
(x A)(x A)^{*} .
$$

These 400 samples of instantaneous power are averaged to get the average output power of the array, $P_{a v}$. This average power represents the power integrated over a P_int $=20 \mu \mathrm{s}$ interval, one iteration of the algorithm. Finally, the gradient components of the output power are computed as in equations (33) and (36),

$$
\frac{\partial P}{\partial w_{m}}=\frac{2 \Re<A_{m} x_{m}, x A>}{w_{m}}
$$

and

$$
\frac{\partial P}{\partial q_{m}}=2 \Im<A_{m} x_{m}, x A>
$$

The term $A_{m} x_{m}$ represents the average signal of 400 samples of output from the $m$ th antenna, $A_{m} x_{m}=N A_{m}+J C A_{m}$ where $N$ is the average noise signal for the current loop iteration. The term $x A$ is the average output signal of the array. Both $A_{m} x_{m}$ and $x A$ are scalars, they are multiplied together and scaled to form $2<A_{m} x_{m}, x A>$. The gradient components are the real (scaled by the reciprocal of each weight magnitude) and imaginary parts of this scaled product. In the Matlab simulation, the gradient components occupy a $2 \times($ Nant-1) matrix

$$
\left[\begin{array}{c}
\frac{\partial P}{\partial w_{2}} \frac{\partial P}{\partial w_{3}} \cdots \frac{\partial P}{\partial w_{N_{a n t}}} \\
\frac{\partial P}{\partial q_{2}} \frac{\partial P}{\partial q_{3}} \cdots \frac{\partial P}{\partial q_{N_{a n t}}}
\end{array}\right] .
$$

The vector of antenna weights is adjusted using the steepest descent algorithm as follows. From equation (27), the amount of weight change is calculated as

$$
\Delta W=-\mu \nabla P=\frac{\left(\text { target power }-P_{a v}\right)}{\nabla P^{T} \nabla P} \nabla P
$$

where target power is taken to be the constant noise floor. $W$ represents the weights as a $2 \times\left(N_{\text {ant }}-1\right)$ vector

$$
\left[\begin{array}{cccc}
w_{2} & w_{3} & \cdots & w_{N_{a n t}} \\
q_{2} & q_{3} \cdots & q_{N_{a n t}}
\end{array}\right] .
$$


Finally, the weight vector is updated as

$$
W \leftarrow W+\Delta W
$$

This completes one iteration of the algorithm. The process repeats until the number of iterations determined by the user entered variable sim_time, has elapsed.

The algorithm is designed to reduce the array output power to within $5 \mathrm{~dB}$ of the target level, noiseffloor, or less. If the output power ever exceeds this level, the average number

of iterations it takes for the algorithm to return the output power to within $5 \mathrm{~dB}$ of the noise_floor level is reported on the output graphs of adapt.m.

The file adapt.m generates 3 output plots: the array output power versus time, the oracle power versus time, and the condition number of $C_{\text {reduced }}$ versus iteration number.

\section{3 motion.m}

If the array platform moves with roll, pitch, or yaw, the file motion.m is called from each iteration of adapt.m to update the phase matrix $C$. As the array rotates, the arrival angles of the jammers change from their original values.

The velocity vector of a jammer with respect to the array is

$$
\vec{v}=\vec{\omega} \times \vec{R}
$$

where $\vec{\omega}$ is the angular velocity of the array

$$
\vec{\omega}=\operatorname{roll} \hat{i}+\operatorname{pitch} \hat{j}+\operatorname{yaw} \hat{k}
$$

and $\vec{R}$ is the vector describing the location of a jammer direction of arrival ( $\phi$ represents azimuth arrival direction, $\theta$ represents elevation arrival direction) expressed in Cartesian coordinates with respect to the center of the array

$$
\vec{R}=\sin \theta \cos \phi \hat{i}+\sin \theta \sin \phi \hat{j}+\cos \theta \hat{k}
$$


The components of $\vec{v}$ are then

$$
\begin{aligned}
\vec{\omega} \times \vec{R}= & (\text { pitch } \cos \theta-\text { yaw } \sin \theta \sin \phi) \hat{i} \\
& +(\text { yaw } \sin \theta \cos \phi-\text { roll } \cos \theta) \hat{j} \\
& +(\text { roll } \sin \theta \sin \phi-\text { pitch } \sin \theta \cos \phi) \hat{k}
\end{aligned}
$$

but the velocity of the array is also the change in time of the arrival directions of the jammers

$$
\vec{v}=d \vec{R} / d t=-\sin \theta \sin \phi \dot{\phi} \hat{i}+\cos \phi \cos \theta \dot{\theta} \hat{i}+\sin \theta \cos \phi \dot{\phi} \hat{j}+\sin \phi \cos \theta \dot{\theta} \hat{j}-\sin \theta \dot{\theta} \hat{k}
$$

Evaluating the $\hat{k}$ components on both sides of $d \vec{R} / d t=\vec{\omega} \times \vec{R}$ leads to the expression for the change in the elevation angle due to roll, pitch, and yaw motion

$$
\dot{\theta}=-\operatorname{roll} \sin \phi+\text { pitch } \cos \phi
$$

Evaluating the $\hat{j}$ components on both sides of $d \vec{R} / d t=\omega \times \vec{R}$ leads to the expression for the change in the azimuth angle

$$
\dot{\phi}=\text { yaw }-\operatorname{roll} \cot \theta \cos \phi-\text { pitch } \cot \theta \sin \phi .
$$

Equations (38) and (39) are the change in jammer arrival angles with respect to time. The file motion.m is called once per iteration of adapt.m and each iteration is P int or 20 $\mu$ seconds in duration. The amounts of change in the jammer arrival directions during an iteration are then $\dot{\phi} \cdot \mathrm{P}_{\_}$int and $\dot{\theta} \cdot \mathrm{P}_{\_}$int. The values of $\phi$ and $\theta$ are updated by the amounts

$$
\phi \leftarrow \phi+\dot{\phi} \cdot P_{-} \text {int }
$$

and

$$
\theta \leftarrow \theta+\dot{\theta} \cdot \text { P_int. }
$$


These new values for $\phi$ and $\theta$ are used to update the components of the phase matrix $C$ for use in the power, gradient, and weight calculations in the next iteration of adapt.m as the array rotates. 


\section{Chapter 6}

\section{Algorithm and Oracle Performance}

The HSB gradient algorithm and oracle performance will be presented for various input scenarios in this chapter. The user can configure many inputs when running the algorithm such as the number of jammers impinging on the array, the jammer arrival angles, the jammer frequencies, the number of peripheral antennas, the array configuration, the input noise level, array motion of roll, pitch, or yaw, and any initial array offset in the roll direction. The figures included in this chapter are representative of the performance of the HSB gradient algorithm for a planar circular array. Various input scenarios are included to show the robustness of the HSB gradient algorithm.

\subsection{Summary of Performance}

This section will provide a brief summary of the performance of the algorithm and the oracle. The performance will be reported for 3 categories: no motion, yaw motion, and full (roll, pitch, and yaw) motion.

The input to the array is modeled as high power jammers and low power noise. The GPS signals are not modeled explicitly, although they would appear similar to the noise. The jammer amplitudes are represented as voltages and the noise amplitude is represented in watts. During each algorithm iteration, the array output power is sampled every $\tau=50$ ns and $\Gamma=400$ of these samples are averaged to represent the average array output power. The averaging interval, one algorithm iteration, is $20 \mu \mathrm{s}$. The gradient of this average output power is calculated and the antenna weights are updated per iteration of the HSB gradient algorithm. 


\subsubsection{No Motion}

The algorithm reduces the array output power to within $5 \mathrm{~dB}$ of the $1 \mathrm{~dB}$ noisefloor more than $99.8 \%$ of the time. The algorithm takes, on average, 10 iterations or $200 \mu$ s to reduce the initial array output power to within $5 \mathrm{~dB}$ of the noisefloor. The fewer the number of jammers, the faster the initial convergence. The performance does not deteriorate with an increasing number of jammers, as long as $N_{\text {jam }}<N_{\text {ant }}$, nor does performance suffer for varying jammer amplitudes, up to $50 \mathrm{~dB}$. The algorithm maintains the output power below the noisefloor after the initial convergence.

The oracle reduces the array output power to within $5 \mathrm{~dB}$ of the $1 \mathrm{~dB}$ noisefloor 99.9999998\% of time when the jammer arrival directions do not result in a confounding configuration or near-confounding configuration (as determined by the condition number of the phase matrix $C_{\text {reduced }}$, section 3.3.2).

\subsubsection{Yaw Motion}

The performance of the algorithm, simulated with yaw motion $\left(500^{\circ} /\right.$ second or less $)$, is only slightly weaker than the results when there is no motion. The algorithm reduces the array output power to within $5 \mathrm{~dB}$ of the $1 \mathrm{~dB}$ noisefloor more than $99.3 \%$ of the time and initial convergence takes, on average, 10 iterations or $200 \mu \mathrm{s}$. The performance does not deteriorate with an increasing number of jammers or increasing yaw rate, as long as $N_{\text {jam }}<N_{a n t}$, and varying jammer amplitudes, up to $50 \mathrm{~dB}$, do not have an adverse effect on performance. When the output power is greater than $5 \mathrm{~dB}$ above the noisefloor, the number of algorithm iterations to reduce the power below this level increases with the number of jammers but is unaffected by the yaw rate and on average is less than 3 iterations or $60 \mu \mathrm{s}$.

The oracle reduces the array output power to within $5 \mathrm{~dB}$ of the $1 \mathrm{~dB}$ noisefloor 99.9999998\% of time when the jammer arrival directions do not result in a confounding configuration or near-confounding configuration. 


\subsubsection{Full Motion}

The performance of the algorithm and oracle with full roll, pitch, and yaw motion mirror the performance of the algorithm and oracle with only yaw motion. The maximum motion rates simulated were $180^{\circ} /$ second for roll, $180^{\circ} /$ second for pitch, and $500^{\circ} /$ second for yaw.

\subsection{Catalog of Output}

The figures in this section show the output of the Matlab file adapt.m which simulates the HSB gradient algorithm. Each figure contains three plots: the array output power due to the HSB gradient algorithm weights, the array output power due to the oracle weights, and the condition number of the phase matrix $C_{\text {reduced }}$. The array used in these simulations consisted of 7 antennas in a planar configuration of 1 central, fixed-weight antenna and 6 peripheral antennas equally spaced around the central antenna at $0^{\circ}, 60^{\circ}, 120^{\circ}, 180^{\circ}, 240^{\circ}, 300^{\circ}$.

\subsubsection{No Motion}

For the following plots no array motion is simulated. The number of jammers incident on the array is indicated in the plot title. The jammers arrive in the plane of the array with elevation angle $\theta=90^{\circ}$ and azimuth angles $\phi=120^{\circ}, 330^{\circ}, 25^{\circ}, 204^{\circ}, 0^{\circ}$, and $108^{\circ}$. The jammer amplitudes are simulated with constant amplitudes of $100 \mathrm{~V}$ (or $40 \mathrm{~dB}$ ). The noise added to the input of the array is 1.25 (or $1 \mathrm{~dB}$ ) and the algorithm attempts to reduce the output power level to this level. The number of iterations simulated is 25000, a duration of 0.5 seconds. As the plots show, both the oracle and algorithm successfully reduce the output power of the array to the noisefloor. 
Table 3. Typical Data for No Motion Cases.

\begin{tabular}{|c|c|c|c|c|c|}
\hline noisefloor $=1 \mathrm{~dB}$ & \multicolumn{5}{|c|}{ No Motion, 25000 Iterations $(.5 \mathrm{sec})$} \\
\hline & \multirow{3}{*}{\multicolumn{2}{|c|}{$\begin{array}{c}\text { Output Power > } \\
\text { Allowed Level }\end{array}$}} & \multirow{3}{*}{\multicolumn{2}{|c|}{$\begin{array}{c}\text { \# iterations to return } \\
\text { below allowed level }\end{array}$}} & Maximum \\
\hline$J_{1}=\cdots=J_{N_{j a m}}$ & & & & & Condition \\
\hline$=40 \mathrm{~dB}$ & & & & & Number \\
\hline \# jammers & algorithm & oracle & algorithm & oracle & \\
\hline 2 & $0.104 \%$ & $1.6 \mathrm{e}-07 \%$ & 3 & $\overline{0}$ & 1.27 \\
\hline 4 & $0.032 \%$ & $1.6 \mathrm{e}-07 \%$ & 9 & 0 & 3.54 \\
\hline 6 & $0.036 \%$ & $1.6 \mathrm{e}-07 \%$ & 10 & 0 & 14.02 \\
\hline
\end{tabular}

Hilbert Space Based Gradient 6-jammer minimization with 7 antennas
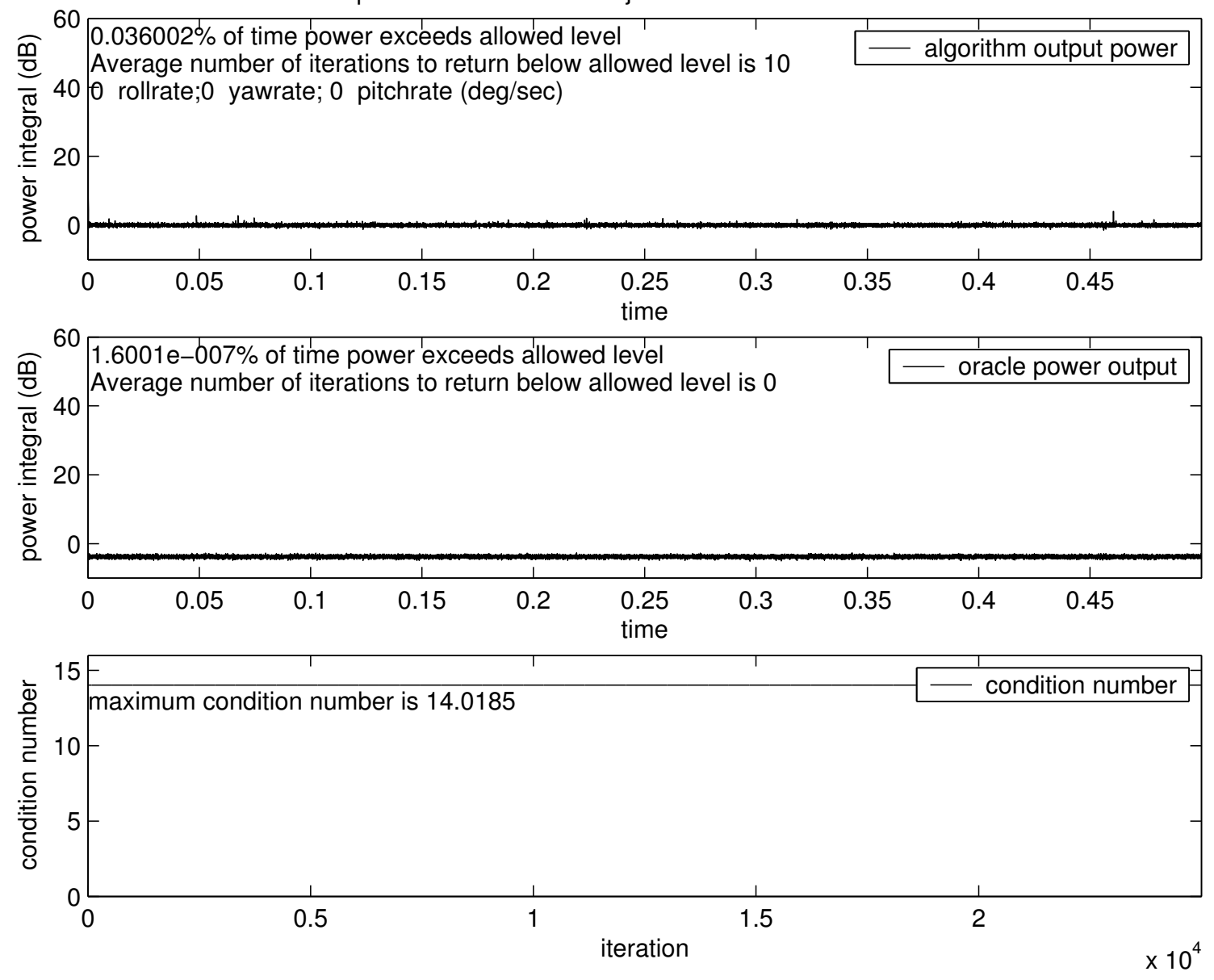

Figure 6. Six Jammers, No Motion. 


\subsubsection{Yaw Motion}

The input conditions for the plots in this section are the same as in the previous section except now the array is simulated with a yaw rate of $360^{\circ} /$ second. The number of jammers incident on the array is indicated in the plot title. The jammers arrive in the plane of the array, $\theta=90^{\circ}$ and initially with $\phi=120^{\circ}, 330^{\circ}, 25^{\circ}, 204^{\circ}, 0^{\circ}$, and $108^{\circ}$. The jammer amplitudes are all equal to $100 \mathrm{~V}(40 \mathrm{~dB})$. The noise added to the input of the array is $1 \mathrm{~dB}$ and the algorithm attempts to reduce the output power to this level. As the plots show, both the oracle and algorithm successfully reduce the output power of the array to the noisefloor.

Note the occurrence of three near-confounding configurations in figure 7. Although the oracle shows difficulty annihilating the jammers at these near-confounding configurations, the HSB gradient algorithm is able to minimize the jammers at these same configurations. Also, when the output power exceeds $5 \mathrm{~dB}$ above the noisefloor, the HSB algorithm requires less weight updates to restore the output power to within $5 \mathrm{~dB}$ of the noisefloor than the oracle, see Table 4 .

Table 4. Typical Data for Yaw Motion Cases.

\begin{tabular}{|c||r|r||r|r||r||}
\hline \multicolumn{1}{|c||}{ noisefloor=1 dB } & \multicolumn{4}{c||}{ Yaw Motion $\left(360^{\circ} / \mathrm{sec}\right), 25000$ Iterations $(.5 \mathrm{sec})$} \\
\hline \multirow{2}{*}{$\begin{array}{c}J_{1}=\cdots=J_{N_{\text {jam }}} \\
=40 \mathrm{~dB}\end{array}$} & $\begin{array}{c}\text { Output Power }> \\
\text { Allowed Level }\end{array}$ & $\begin{array}{c}\text { \# iterations to return } \\
\text { below allowed level }\end{array}$ & $\begin{array}{c}\text { Maximum } \\
\text { Condition } \\
\text { Number }\end{array}$ \\
\hline \# jammers & algorithm & oracle & algorithm & oracle & \\
\hline 2 & $0.7 \%$ & $1.6 \mathrm{e}-07 \%$ & 2 & 0 & 3.13 \\
\hline 4 & $0.504 \%$ & $1.6 \mathrm{e}-07 \%$ & 3 & 0 & 4.84 \\
\hline 6 & $0.564 \%$ & $.924 \%$ & 2 & 34 & 85590.32 \\
\hline
\end{tabular}


Hilbert Space Based Gradient 6-jammer minimization with 7 antennas
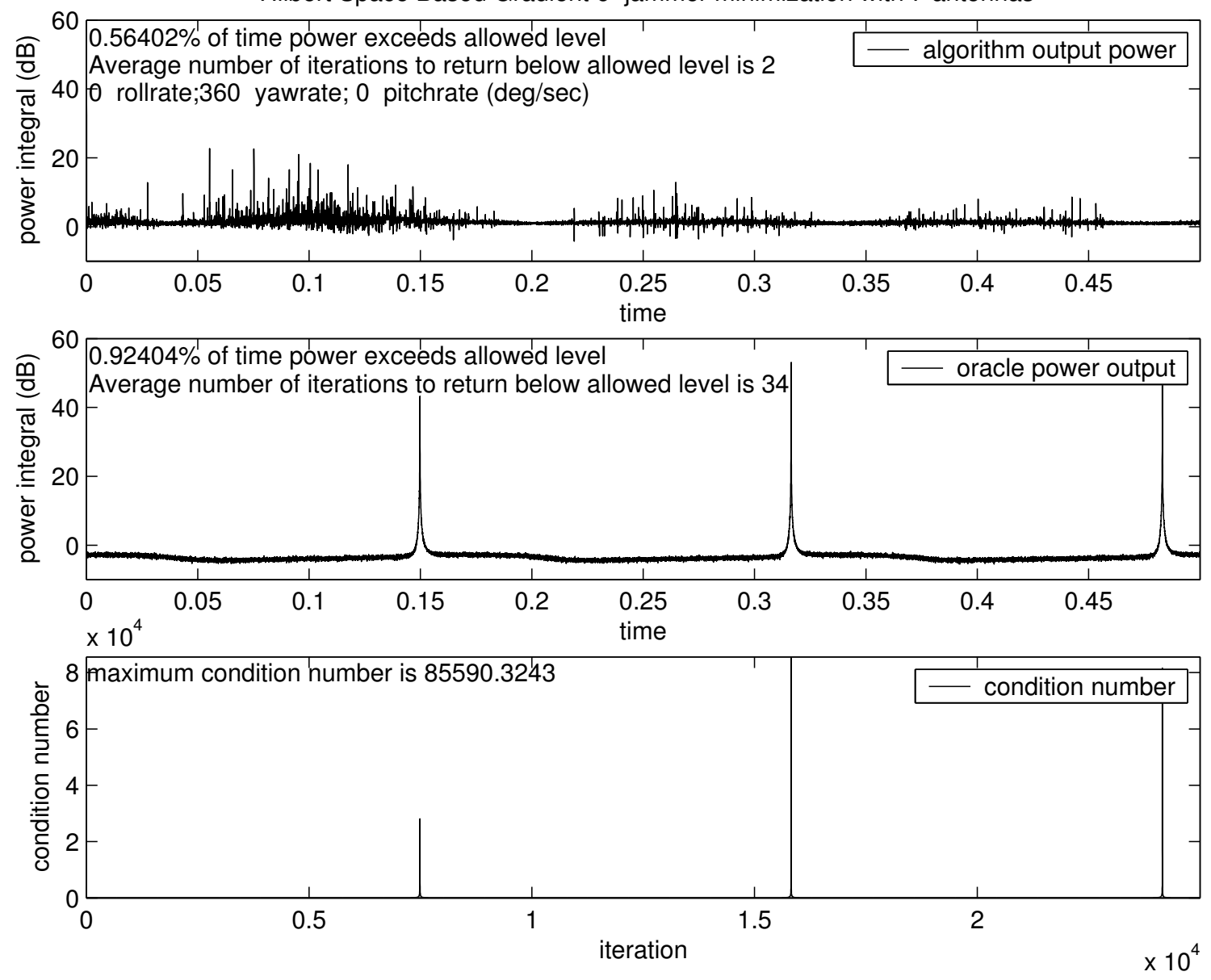

Figure 7. Six Jammers, Yaw Motion, Near Confounding Configuration. 


\subsubsection{Different Jammer Amplitude Levels}

The input conditions for the plots in this section are similar to those of the previous section except that now the jammer amplitudes are at different levels. As noted (section 4.4), this does not affect the oracle solution. The jammers arrive in the plane of the array as before, $\theta=90^{\circ}$ and $\phi=120^{\circ}, 330^{\circ}, 25^{\circ}, 204^{\circ}, 0^{\circ}$, and $108^{\circ}$ but now the jammer amplitude voltages are $316,31.6,100,10,100,316(50 \mathrm{~dB}, 30 \mathrm{~dB}, 40 \mathrm{~dB}, 20 \mathrm{~dB}, 40 \mathrm{~dB}, 50 \mathrm{~dB})$. The array is simulated with and without motion; this is indicated in the plot caption.

Table 5. Typical Data for No Motion Cases with Different Jammer Amplitudes.

\begin{tabular}{|l||r|r||r|r||r||}
\hline \multicolumn{1}{|l||}{ noisefloor=1 dB } & \multicolumn{4}{c||}{ No Motion, 25000 Iterations (.5 sec) } \\
\hline $\begin{array}{l}\text { different jammer } \\
\text { amplitudes }\end{array}$ & $\begin{array}{r}\text { Output Power }> \\
\text { Allowed Level }\end{array}$ & $\begin{array}{l}\text { \# iterations to return } \\
\text { below allowed level }\end{array}$ & $\begin{array}{l}\text { Maximum } \\
\text { Condition } \\
\text { Number }\end{array}$ \\
\hline$\#$ jammers & algorithm & oracle & algorithm & oracle & \\
\hline $2(50,30 \mathrm{~dB})$ & $0.244 \%$ & $1.6 \mathrm{e}-07 \%$ & 2 & 0 & 1.27 \\
\hline $\begin{array}{l}4(50,30,40,20 \\
\mathrm{dB})\end{array}$ & $0.084 \%$ & $1.6 \mathrm{e}-07 \%$ & 3 & 0 & 3.54 \\
\hline $\begin{array}{l}6(50,30,40,20, \\
40,50 \mathrm{~dB})\end{array}$ & $0.128 \%$ & $1.6 \mathrm{e}-07 \%$ & 3 & 0 & 14.02 \\
\hline
\end{tabular}

Table 6. Typical Data for Yaw Motion Cases with Different Jammer Amplitudes.

\begin{tabular}{|l||r|r||r|r||r||}
\hline \multicolumn{1}{|l||}{ noisefloor $=1 \mathrm{~dB}$} & \multicolumn{3}{c||}{ Yaw Motion $\left(360^{\circ} / \mathrm{sec}\right), 25000$ Iterations $(.5 \mathrm{sec})$} \\
\hline \multicolumn{1}{l}{$\begin{array}{l}\text { different jammer } \\
\text { amplitudes }\end{array}$} & \multicolumn{2}{|c||}{$\begin{array}{l}\text { Output Power }> \\
\text { Allowed Level }\end{array}$} & $\begin{array}{l}\text { \# iterations to return } \\
\text { below allowed level }\end{array}$ & $\begin{array}{l}\text { Maximum } \\
\text { Condition } \\
\text { Number }\end{array}$ \\
\hline$\#$ jammers & algorithm & oracle & algorithm & oracle & \\
\hline $2(50,30 \mathrm{~dB})$ & $1.956 \%$ & $1.6 \mathrm{e}-07 \%$ & 2 & 0 & 3.13 \\
\hline $\begin{array}{l}4(50,30,40,20 \\
\mathrm{dB})\end{array}$ & $0.988 \%$ & $1.6 \mathrm{e}-07 \%$ & 2 & 0 & 4.84 \\
\hline $\begin{array}{l}6(50,30,40,20, \\
40,50 \mathrm{~dB})\end{array}$ & $3.088 \%$ & $.924 \%$ & 3 & 34 & 85590 \\
\hline
\end{tabular}


Hilbert Space Based Gradient 4-jammer minimization with 7 antennas
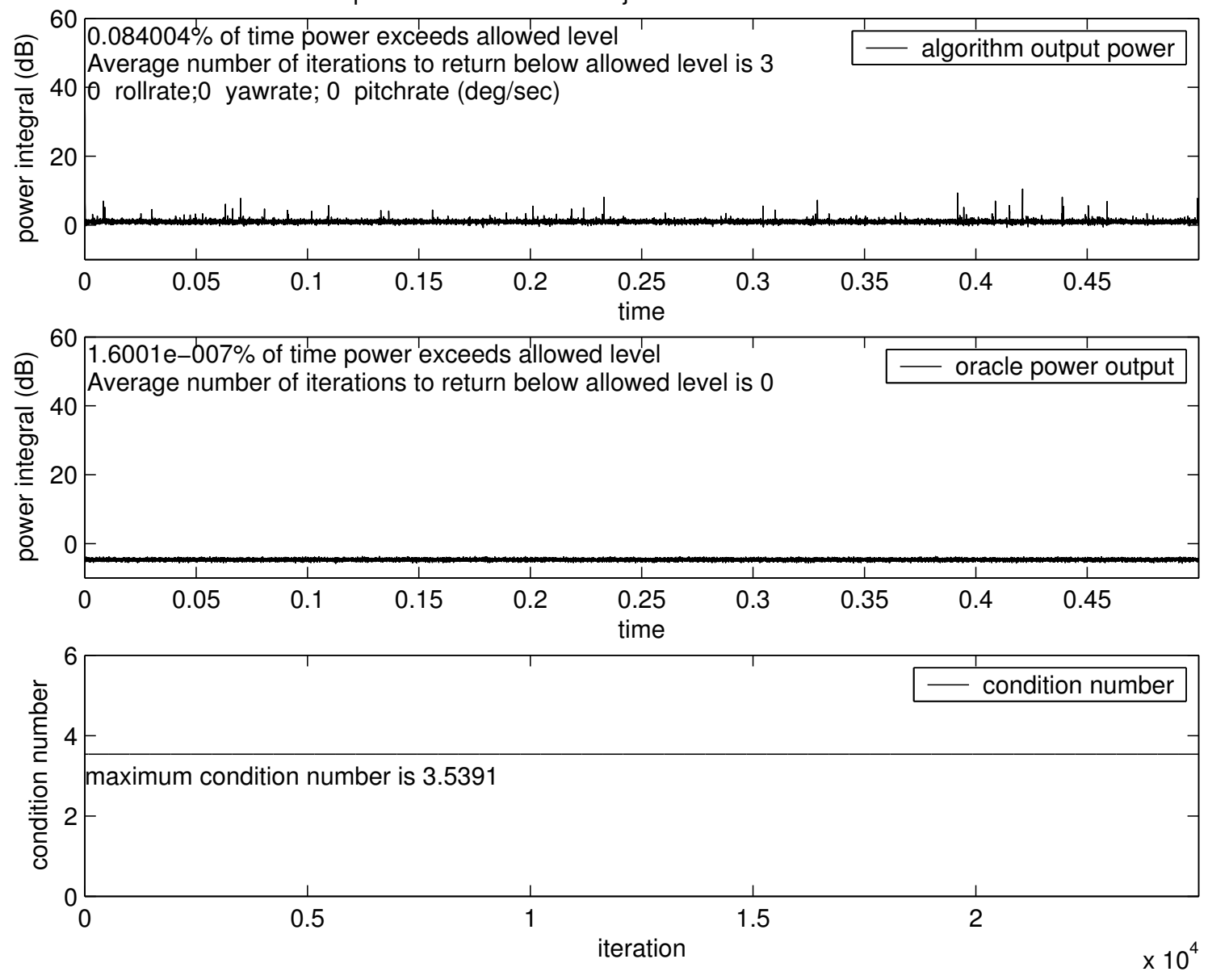

Figure 8. Four Jammers 50 dB, 30 dB, 40 dB, 20 dB; No Motion. 
Hilbert Space Based Gradient 6-jammer minimization with 7 antennas
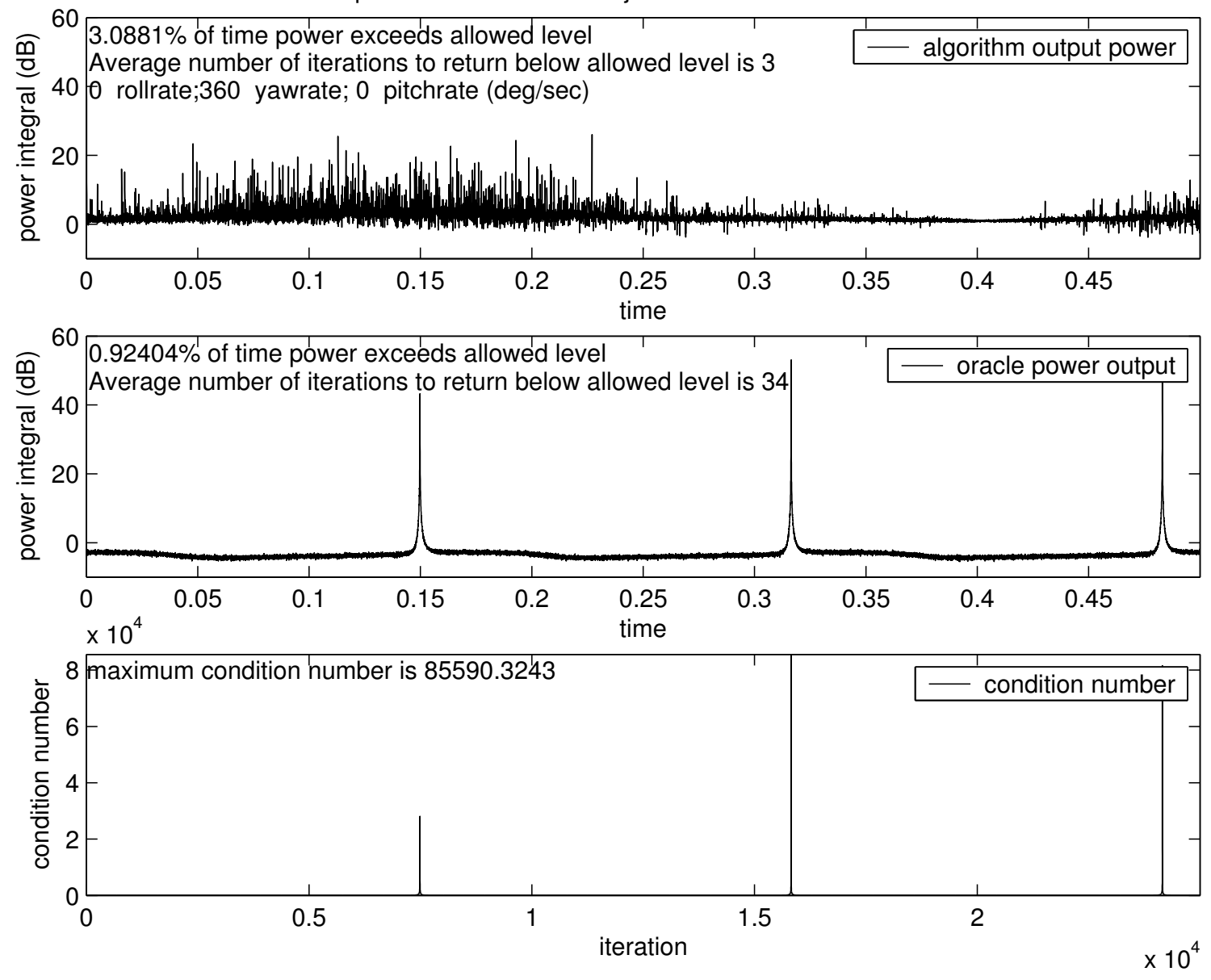

Figure 9. Six Jammers 50, 30, 40, 20, 40, 50 dB; Yaw Motion. 


\subsubsection{Full Motion}

The array is simulated with roll $=20^{\circ} /$ second, pitch $=50^{\circ} /$ second, and yaw $=180^{\circ} /$ second. The jammers arrive with different amplitudes, indicated in the plot caption and the arrival directions as in previous sections, $\theta=90^{\circ}$ and $\phi=120^{\circ}, 330^{\circ}, 25^{\circ}, 204^{\circ}, 0^{\circ}$, and $108^{\circ}$. The noisefloor the algorithm attempts to reach is $1 \mathrm{~dB}$.

Table 7. Typical Data for Full Motion Cases.

\begin{tabular}{|l||r|r||r|r||r||}
\hline \multicolumn{1}{|l||}{ noisefloor=1 dB } & \multicolumn{4}{c||}{ Full Motion, 25000 Iterations (.5 sec) } \\
\hline \multicolumn{1}{l}{$\begin{array}{l}\text { different jammer } \\
\text { amplitudes }\end{array}$} & $\begin{array}{r}\text { Output Power }> \\
\text { Allowed Level }\end{array}$ & $\begin{array}{l}\text { \# iterations to return } \\
\text { below allowed level }\end{array}$ & $\begin{array}{r}\text { Maximum } \\
\text { Condition } \\
\text { Number }\end{array}$ \\
\hline$\#$ jammers & algorithm & oracle & algorithm & oracle & \\
\hline $2(50,30 \mathrm{~dB})$ & $0.784 \%$ & $1.6 \mathrm{e}-07 \%$ & 2 & 0 & 3.063 \\
\hline $\begin{array}{l}4(50,30,40,20 \\
\mathrm{dB})\end{array}$ & $0.516 \%$ & $1.6 \mathrm{e}-07 \%$ & 2 & 0 & 4.831 \\
\hline $\begin{array}{l}6(50,30,40,20, \\
40,50 \mathrm{~dB})\end{array}$ & $1.664 \%$ & $0.792 \%$ & 2 & 41 & 62644 \\
\hline
\end{tabular}


Hilbert Space Based Gradient 4-jammer minimization with 7 antennas
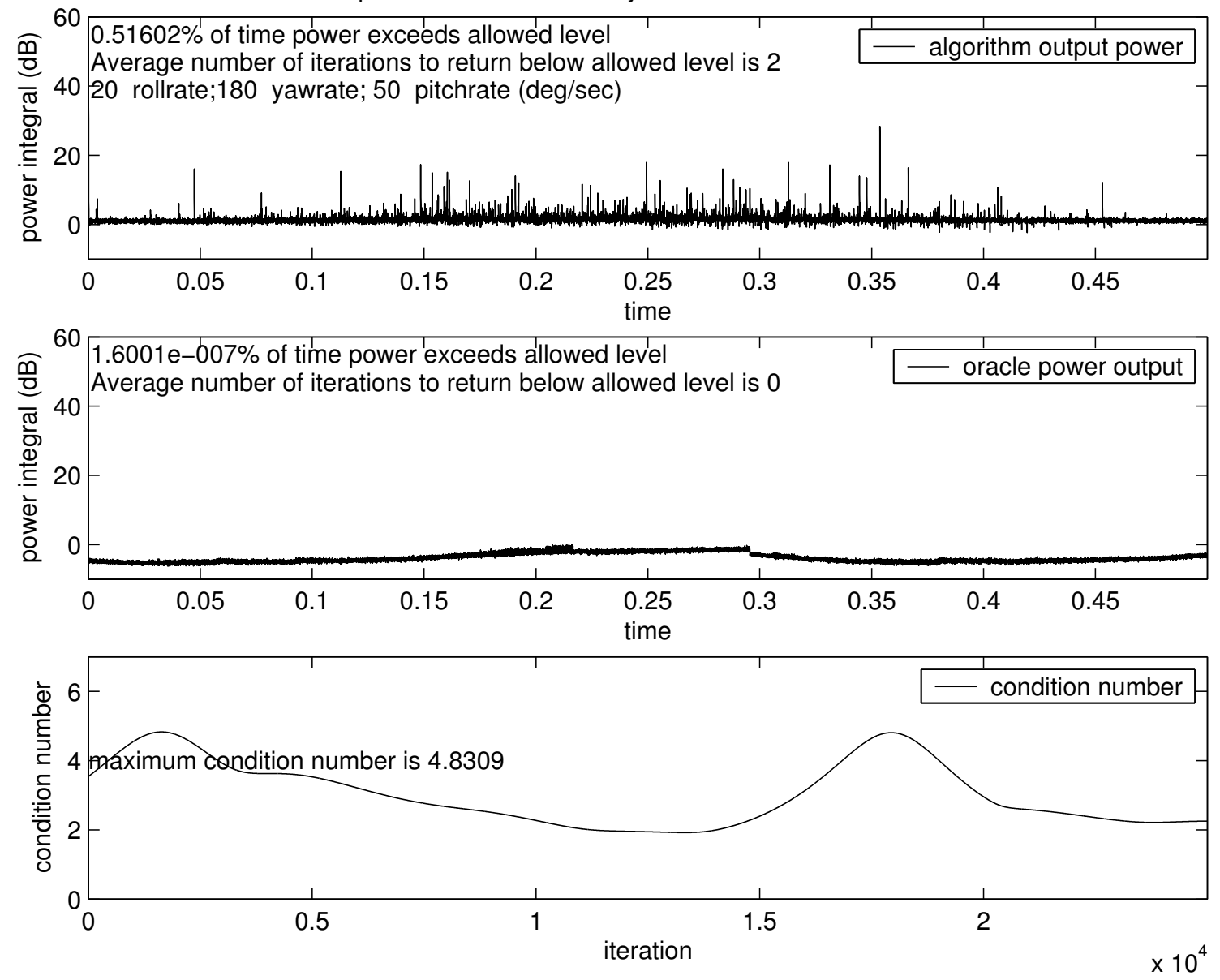

Figure 10. Six Jammers 50, 30, 40, 20, 40, 50 dB; Full Motion. 


\subsection{A Note on Out-of-Plane Jammers}

For a planar array, an out-of-plane jammer is mathematically equivalent to an in-plane jammer at a lower frequency. A jammer at wavelength $\lambda_{1}$, arriving at the array from a direction $(\phi, \theta)$ generates an array output

$$
J C A=J_{1} e^{i \omega_{1} t}\left[e^{i \frac{2 \pi}{\lambda_{1}} \vec{a}_{1} \cdot \vec{j}_{1}} \cdots e^{i \frac{2 \pi}{\lambda_{1}} \vec{a}_{N_{a n t}} \cdot \vec{j}_{1}}\right]\left[\begin{array}{c}
1 \\
w_{2} e^{i q_{2}} \\
\vdots \\
w_{N_{a n t}} e^{i q_{N_{a n t}}}
\end{array}\right]
$$

Recall, from equation (37), that the array output from this jammer impinging on the $m$ th antenna is

$$
J_{1} e^{i \frac{2 \pi}{\lambda_{1}} r \cos \left(\phi-\gamma_{m}\right) \sin \theta} w_{m} e^{i q_{m}}
$$

We can define a new, longer wavelength $\lambda^{\prime}=\lambda_{1} / \sin \theta$ so that the jammer signal at the array output is equivalent to

$$
J_{1} e^{i \frac{2 \pi}{\lambda^{\prime}} r \cos \left(\phi-\gamma_{m}\right)} w_{m} e^{i q_{m}}
$$

in the plane of the array $\left(\theta=90^{\circ}\right)$.

\subsection{Noise Immunity}

The objective of this section is to investigate how far above the noisefloor any additional input noise can be before the algorithm fails. Recall the desired GPS signals are on the order of or below the noisefloor but are spread spectrum modulated. As long as the array radiation pattern nulls do not coincide with the arrival directions of the GPS signals, the latter will pass through the array and be demodulated accurately. If the input noise and jammer amplitudes are on the order of the noisefloor or are below the noisefloor, the algorithm has nothing to do; the output is already at the noisefloor. The algorithm adaptively reduces the output power of the array only when the input to the array, assumed to be primarily 
from undesired jammer signals, is above the noisefloor. Various cases were run to test both the HSB gradient algorithm and the oracle for noise immunity.

The array used in these simulations consisted of 7 antennas in a planar configuration of 1 central, fixed-weight antenna and 6 peripheral antennas at $\gamma=0^{\circ}, 60^{\circ}, 120^{\circ}, 180^{\circ}, 240^{\circ}$,$300^{\circ}$. The jammers arrive in the plane of the array, $\theta=90^{\circ}$ and $\phi=120^{\circ}, 330^{\circ}, 25^{\circ}, 204^{\circ}, 0^{\circ}$, and $108^{\circ}$ with different amplitudes of $316(50 \mathrm{~dB}), 31.6(30 \mathrm{~dB}), 100(40 \mathrm{~dB}), 10(20 \mathrm{~dB}), 100$ $(40 \mathrm{~dB}), 316(50 \mathrm{~dB})$. The array is in motion with yaw $=360^{\circ} /$ second. Three categories of cases were run in which the algorithm attempted to reduce the array output power to within $5 \mathrm{~dB}$ of noisefloors of $0 \mathrm{~dB}, 1 \mathrm{~dB}$, and $3 \mathrm{~dB}$. The noise added as input to the array above the noisefloor is indicated in the tables. The results show that the algorithm is quite sensitive to noise added as input above the noisefloor. Failure is said to occur when the algorithm cannot reduce the output power below the noisefloor. An interesting feature is that the algorithm has a failure rate that decreases with the number of incident jammers. With more nulls in the array radiation pattern, more of the input noise can be attenuated.

Table 8. Algorithm Failure Rate with Increasing Noise Levels (noisefloor $=0 \mathrm{~dB}$ ).

\begin{tabular}{|c||r|r||r|r||r|r||}
\hline \multicolumn{1}{|c||}{ noisefloor $=0 \mathrm{~dB}$} & \multicolumn{3}{c||}{ Njam } \\
\hline \multicolumn{1}{|c||}{} & \multicolumn{2}{c||}{2} & \multicolumn{2}{c||}{4} & \multicolumn{2}{c||}{6} \\
\hline added noise & algorithm & oracle & algorithm & oracle & algorithm & oracle \\
\hline $5 \mathrm{~dB}$ & $36.113 \%$ & $1.6 \mathrm{e}-07 \%$ & $17.373 \%$ & $1.6 \mathrm{e}-07 \%$ & $23.345 \%$ & $100 \%^{*}$ \\
\hline $6 \mathrm{~dB}$ & $51.85 \%$ & $1.6 \mathrm{e}-07 \%$ & $31.873 \%$ & $1.6 \mathrm{e}-07 \%$ & $37.213 \%$ & $100 \%^{*}$ \\
\hline $8 \mathrm{~dB}$ & $80.563 \%$ & $96.172 \%$ & $59.586 \%$ & $96.172 \%$ & $62.342 \%$ & $100 \%^{*}$ \\
\hline
\end{tabular}

* indicates near-confounding configuration

Table 9. Algorithm Failure Rate with Increasing Noise Levels (noisefloor=1 dB).

\begin{tabular}{|c||r|r||r|r||r|r||}
\hline \multicolumn{1}{|c||}{ noisefloor=1 dB } & \multicolumn{3}{c||}{ Njam } \\
\hline \multicolumn{1}{|c||}{} & \multicolumn{2}{c||}{2} & \multicolumn{2}{c||}{4} & \multicolumn{2}{c||}{6} \\
\hline added noise & algorithm & oracle & algorithm & oracle & algorithm & oracle \\
\hline $5 \mathrm{~dB}$ & $20.709 \%$ & $1.6 \mathrm{e}-07 \%$ & $9.692 \%$ & $1.6 \mathrm{e}-07 \%$ & $14.633 \%$ & $100 \%^{*}$ \\
\hline $6 \mathrm{~dB}$ & $35.153 \%$ & $1.6 \mathrm{e}-07 \%$ & $17.613 \%$ & $1.6 \mathrm{e}-07 \%$ & $22.677 \%$ & $100 \%^{*}$ \\
\hline $8 \mathrm{~dB}$ & $71.207 \%$ & $8.976 \%$ & $46.722 \%$ & $8.976 \%$ & $48.794 \%$ & $100 \%^{*}$ \\
\hline
\end{tabular}


Table 10. Algorithm Failure Rate with Increasing Noise Levels (noisefloor $=3 \mathrm{~dB}$ ).

\begin{tabular}{|c||r|r||r|r||r|r||}
\hline \multicolumn{1}{|c||}{ noisefloor=3 dB } & \multicolumn{3}{c||}{ Njam } \\
\hline \multicolumn{1}{|c||}{} & \multicolumn{2}{c||}{2} & \multicolumn{2}{c||}{4} & \multicolumn{2}{||}{6} \\
\hline added noise & algorithm & oracle & algorithm & oracle & algorithm & oracle \\
\hline $5 \mathrm{~dB}$ & $5.284 \%$ & $1.6 \mathrm{e}-7 \%$ & $2.276 \%$ & $1.6 \mathrm{e}-7 \%$ & $4.548 \%$ & $99.672 \%^{*}$ \\
\hline $6 \mathrm{~dB}$ & $10.64 \%$ & $1.6 \mathrm{e}-7 \%$ & $4.828 \%$ & $1.6 \mathrm{e}-7 \%$ & $7.368 \%$ & $99.708 \%^{*}$ \\
\hline $8 \mathrm{~dB}$ & $34.381 \%$ & $1.6 \mathrm{e}-7 \%$ & $16.621 \%$ & $1.6 \mathrm{e}-7 \%$ & $20.705 \%$ & $99.98 \%^{*}$ \\
\hline $10 \mathrm{~dB}$ & $66.139 \%$ & $7.12 \%$ & $45.062 \%$ & $7.12 \%$ & $48.41 \%$ & $100 \%^{*}$ \\
\hline
\end{tabular}

* indicates near-confounding configuration 


\section{Chapter 7}

\section{Summary and Future Work Directions}

The main contributions of this work are as follows.

The exact components of the gradient are calculated using inner products of signals readily available at the individual antenna outputs and at the array output. The computation is fast and all of the gradient components are calculated per iteration.

The condition number is used to indicate the likelihood of the existence of the optimal antenna weights. A high condition number indicates a confounding configuration; one without stable oracle weights.

The ability to construct confounding configurations is presented. Research funding is being pursued to study other methods to construct confounding configurations as well as the geometric interpretations, if any, of them.

The oracle solution calculates the exact antenna weights to null the jammer component of the array output. The weights depend only upon the present configuration of antennas and jammers and so provide a check of the effectiveness of weights at any configuration from any adaptive algorithm.

The HSB gradient algorithm, implemented in Matlab, successfully minimizes the power output of the array.

Areas for future work concern the investigation of an adaptive accumulation time for the calculation of the average array output power. As the array motion increases, the accumulation of instantaneous array output power measurements must decrease to capture the dynamic information of the non-stationary array environment. 


\section{References}

[1] Robert E. Collin. Antennas and Radiowave Propagation, section 3.6, pages 107-121. McGraw-Hill Series in Electrical Engineering. McGraw-Hill, Inc., New York, New York, 1985.

[2] Biswa Nath Datta. Numerical Linear Algebra and Applications, chapter 7, pages 316324. Brooks/Cole Publishing Company, Pacific Grove, California, 1996.

[3] Biswa Nath Datta. Numerical Linear Algebra and Applications, section 7.9, pages 348-351. Brooks/Cole Publishing Company, Pacific Grove, California, 1996.

[4] Paul G. Flikkema. Spread spectrum techniques for wireless communications. IEEE Signal Processing Magazine, 14(3):26-28, May 1997.

[5] Anton Gecan, Paul G. Flikkema, and Arthur David Snider. Jammer cancellation with adaptive arrays for gps signals. IEEE Southeastcon Conference Proceedings, pages 320-323, 1996.

[6] Anton S. Gecan and Michael D. Zoltowski. Power minimization techniques for gps null steering antennas. preprint, 1995.

[7] Jr. J. J. Spilker. Gps signal structure and performance characteristics. Navigation, 25(2):121-146, July 1978.

[8] Harold J. Larson. Introduction to Probability Theory and Statistical Inference, section 5.4, pages 178-179. Wiley Series in Probability and Mathematical Statistics. John Wiley and Sons, Inc., New York, New York, 1969.

[9] John Litva and Titus Kwok-Yeung Lo. Digital Beamforming in Wireless Communications, section 2.4, pages 28-34. The Artech House Mobile Communications Series. Artech House, Inc., Norwood, Massachusetts, 1996.

[10] Robert A. Monzingo and Thomas W. Miller. Introduction to Adaptive Arrays, section 3.3, page 89. A Wiley-Interscience Publication. John Wiley and Sons, New York, 1980.

[11] Robert A. Monzingo and Thomas W. Miller. Introduction to Adaptive Arrays, section 4.2, pages 162-178. A Wiley-Interscience Publication. John Wiley and Sons, New York, 1980 .

[12] Robert A. Monzingo and Thomas W. Miller. Introduction to Adaptive Arrays, chapter 5, pages 217-292. A Wiley-Interscience Publication. John Wiley and Sons, New York, 1980. 
[13] Roger L. Peterson, Rodger E. Ziemer, and David E. Borth. Introduction to Spread Spectrum Communications, section 2.3, 3.3, pages 52-60, 113-115. Prentice-Hall, Inc., Upper Saddle River, New Jersey, 1995.

[14] John G. Proakis. Digital Communications, section 13.2, pages 698-699,707-709. McGraw-Hill Series in Electrical and Computer Engineering. Communications and Signal Processing. McGraw-Hill, Inc., New York, New York, third edition, 1995.

[15] Jr. R. T. Compton. The power-inversion adaptive array: Concept and performance. IEEE Transactions on Aerospace and Electronic Systems, 15(6):803-814, November 1979.

[16] Gilbert Strang. Introduction to Linear Algebra, section 3.3, 9.2, pages 125-136,386-388. Wellesley-Cambridge Press, Wellesley, Massachusetts, 1993. 
Appendices 


\section{Appendix A: Symbol Glossary}

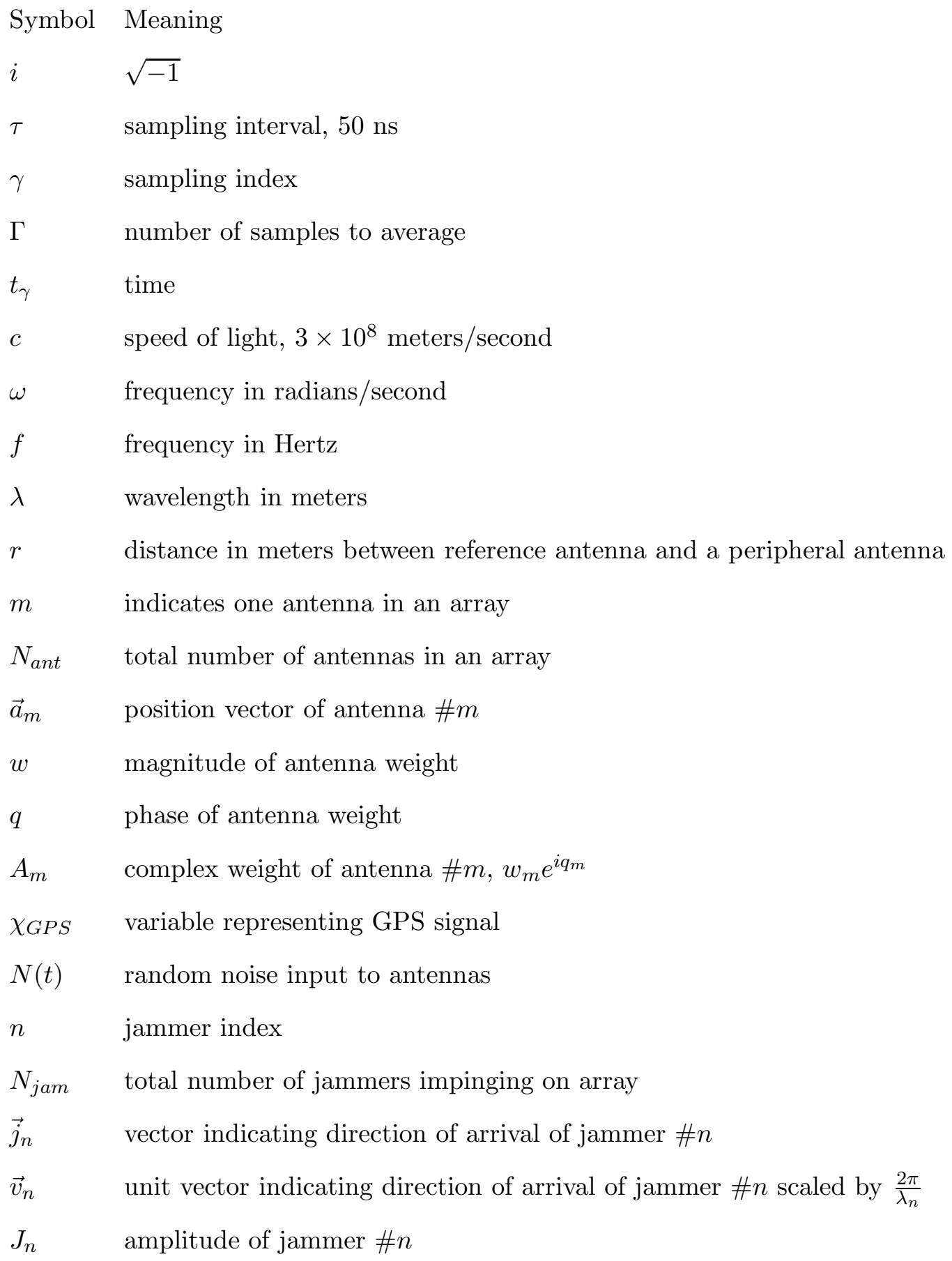


Appendix A (Continued)

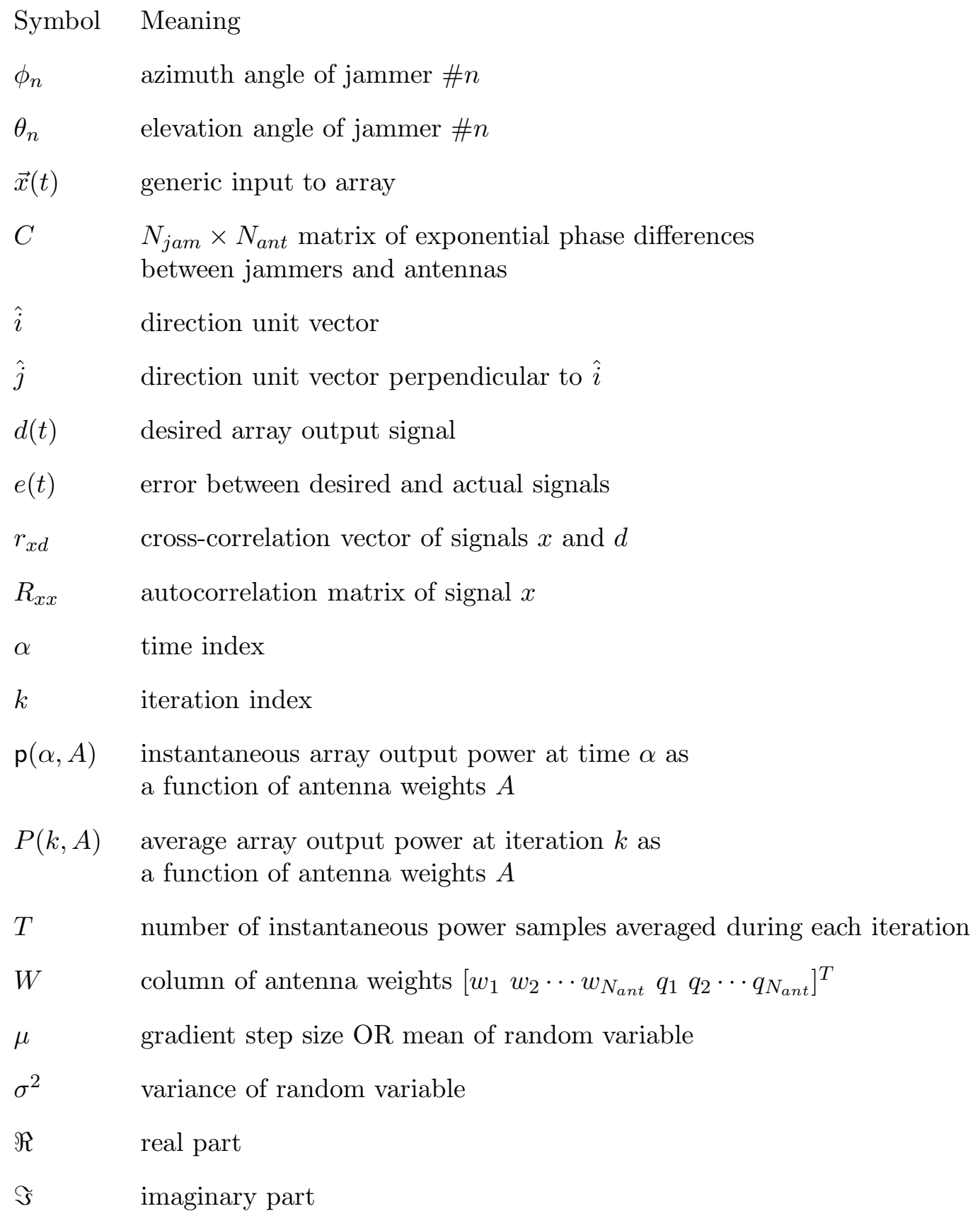




\section{Appendix B: Matlab Source Code}

\section{setup.m}

$\%$ This script is a tool for initializing the adapt simulation.

$\% \quad$ Not all parameters listed herein are used in any given version

$\%$ of the simulation

$\% \quad$ September 24, 2004

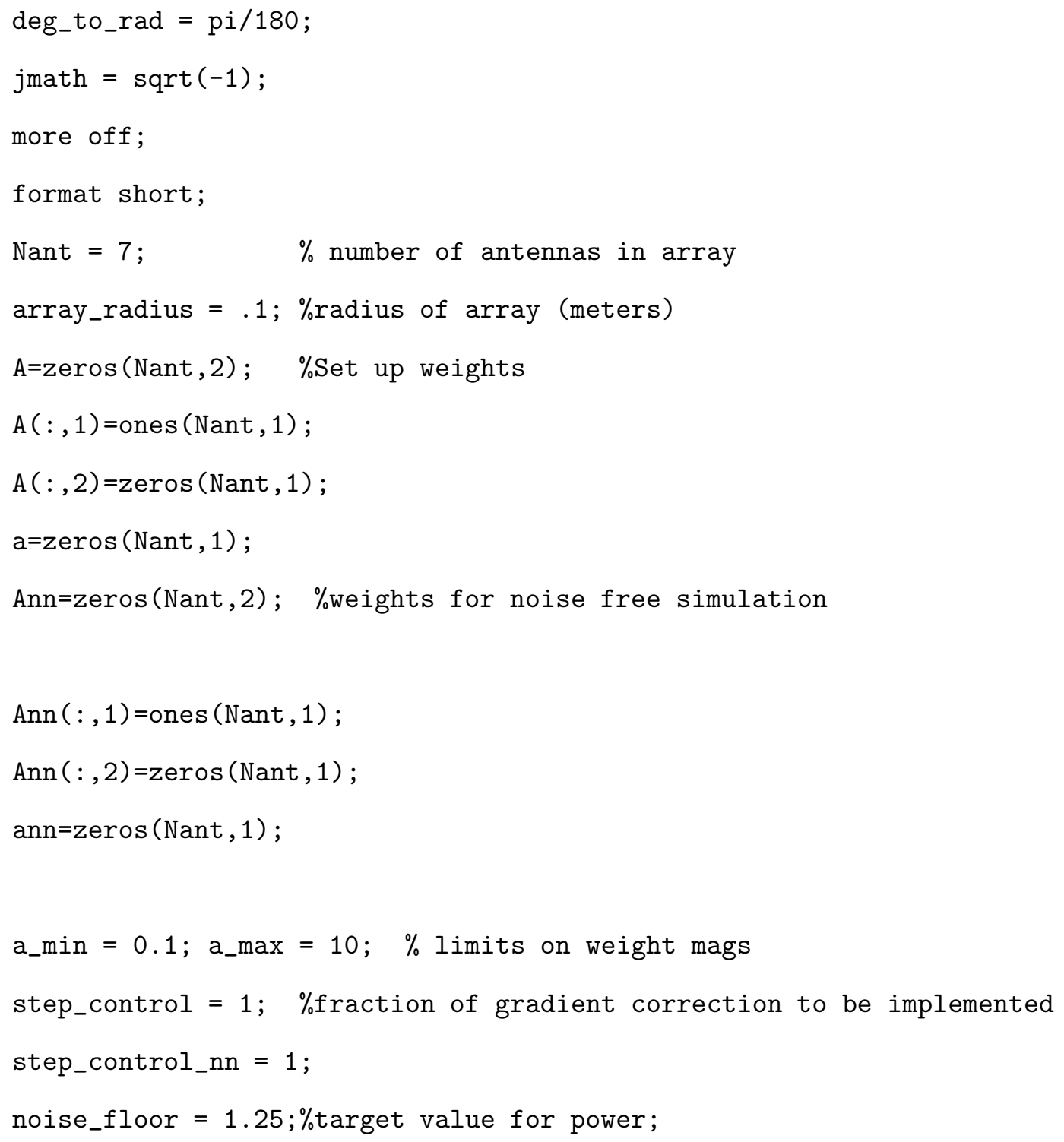


Appendix B (Continued)

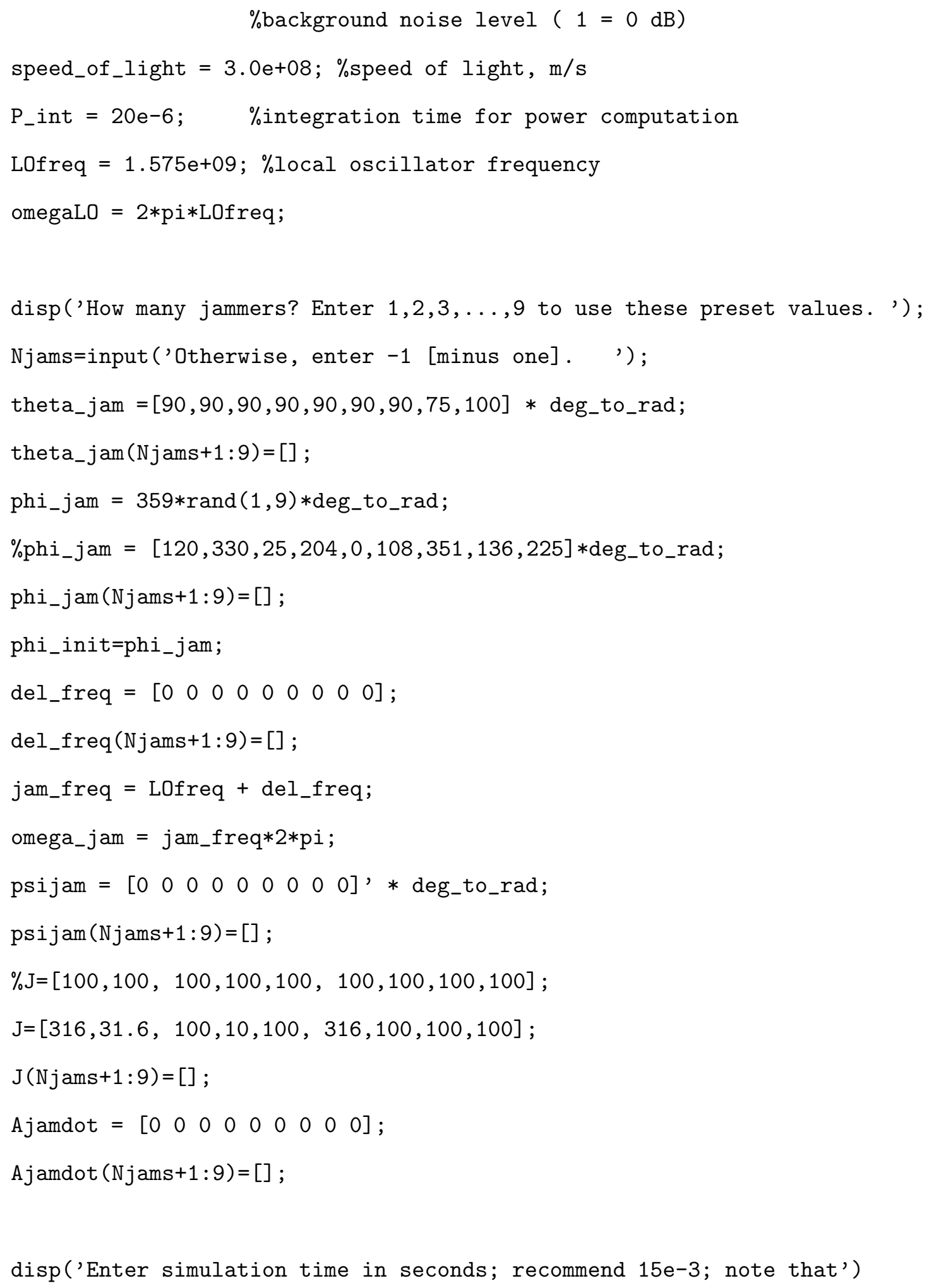


Appendix B (Continued)

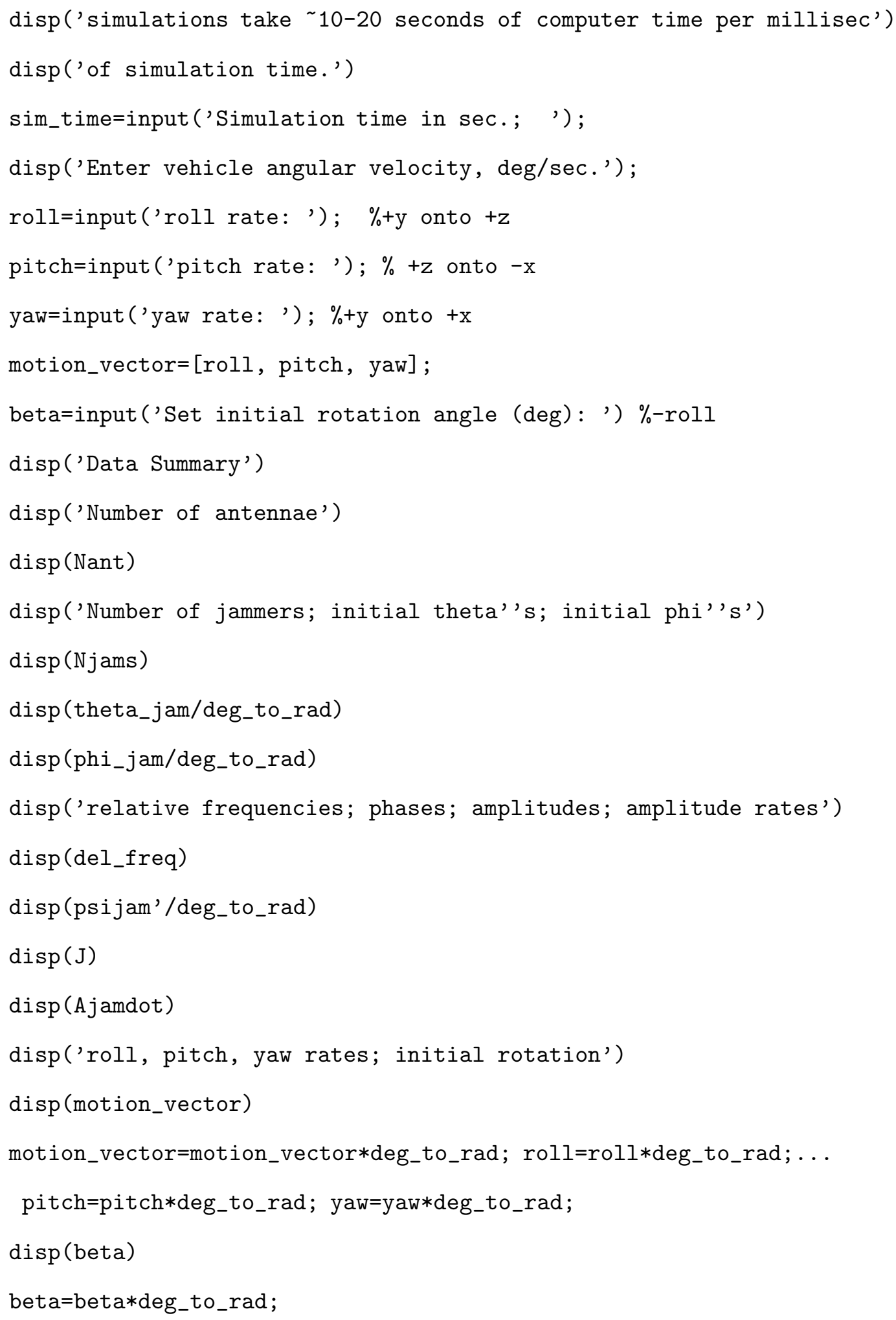


Appendix B (Continued)

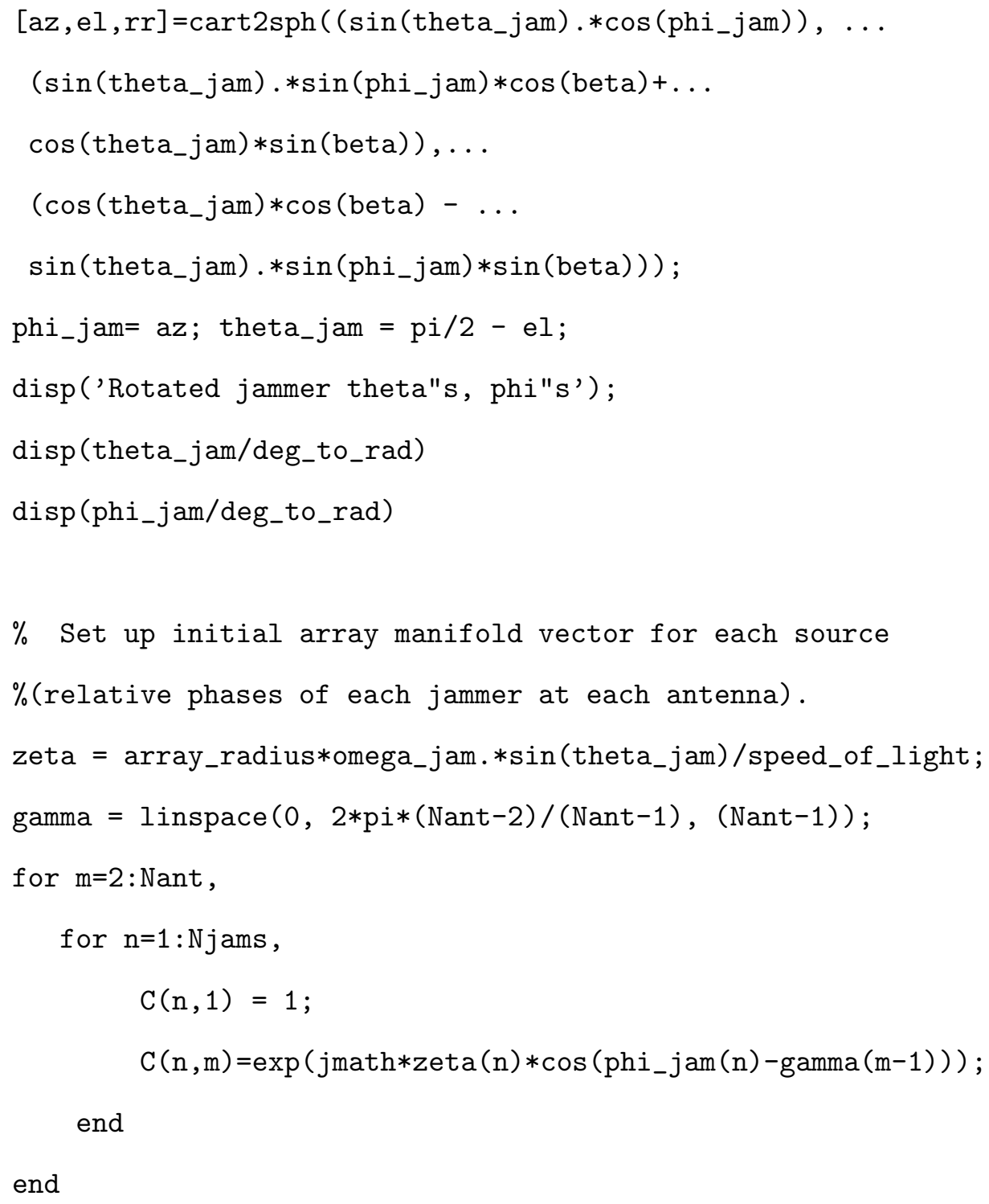


Appendix B (Continued)

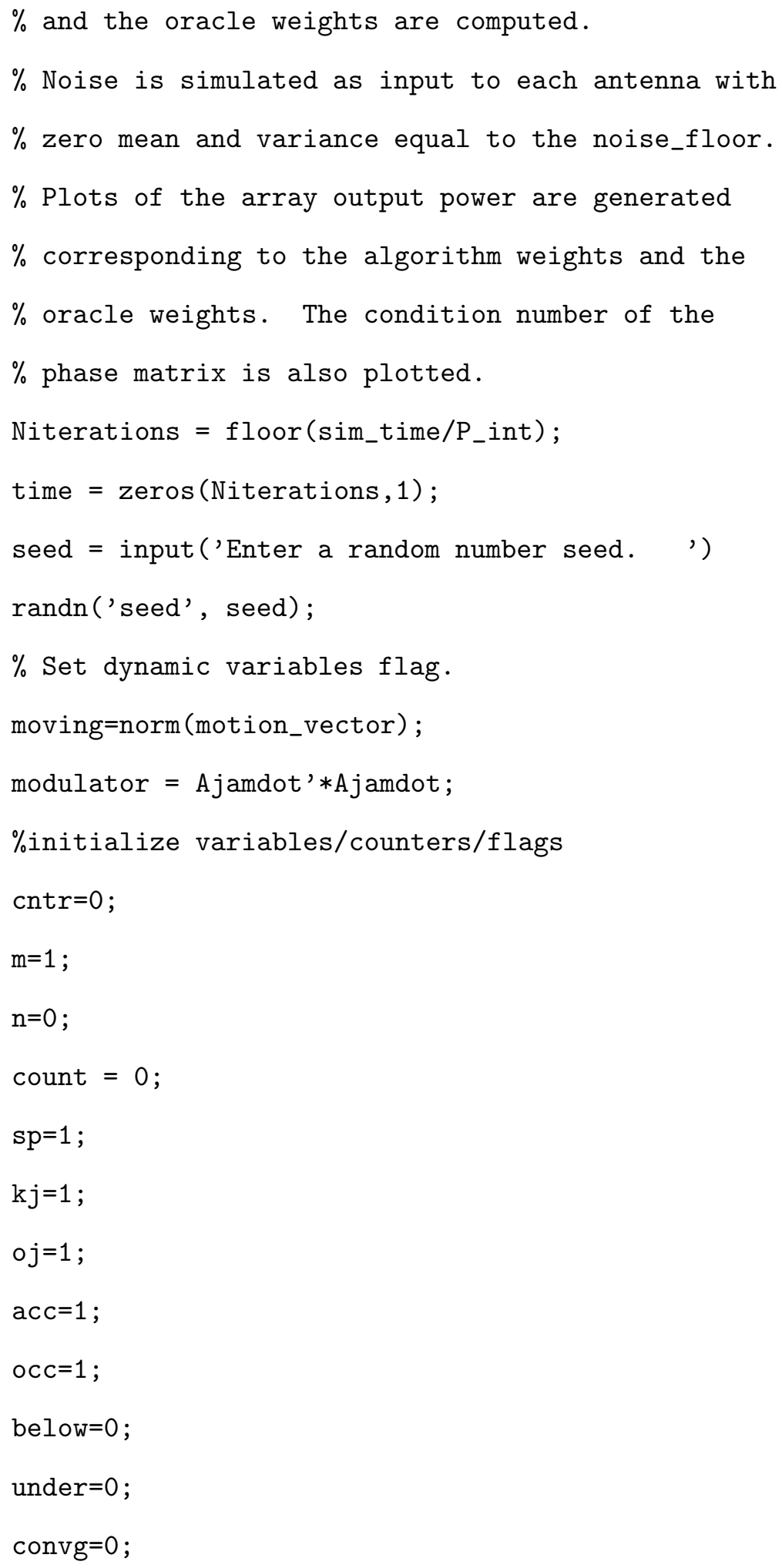


Appendix B (Continued)

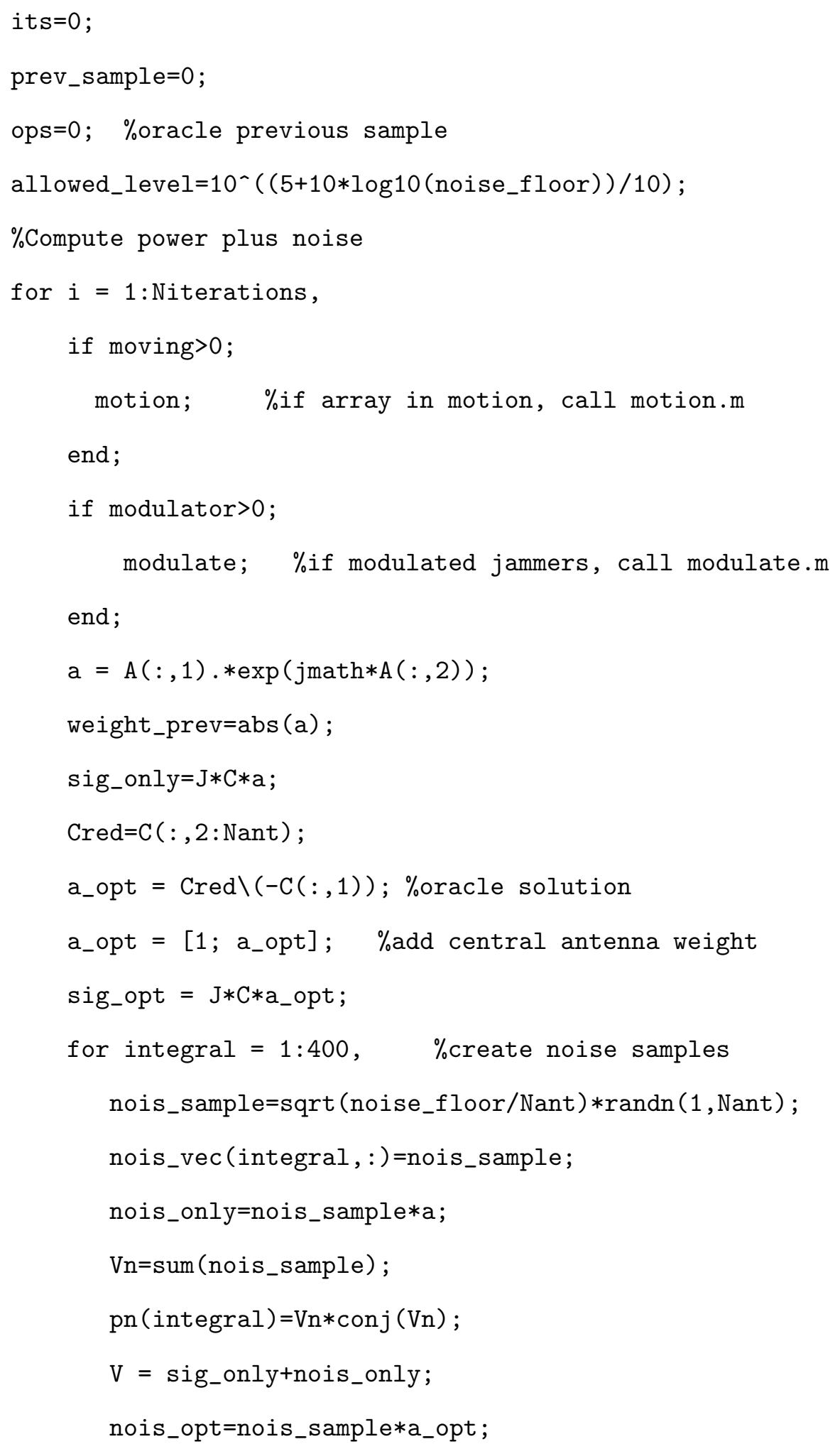


Appendix B (Continued)

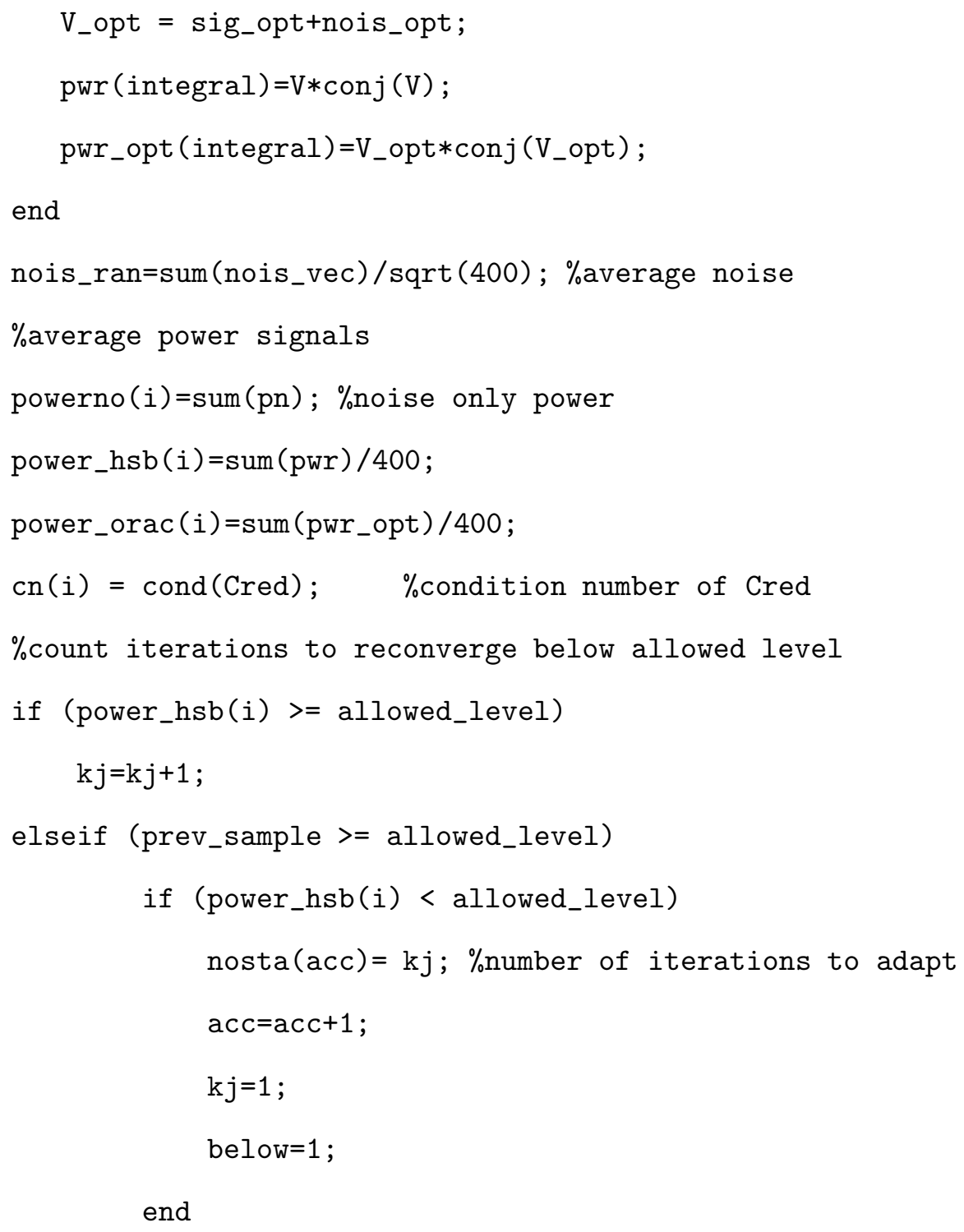

end

if (below $==0$ ) \%power never went below allowed level 
Appendix B (Continued)

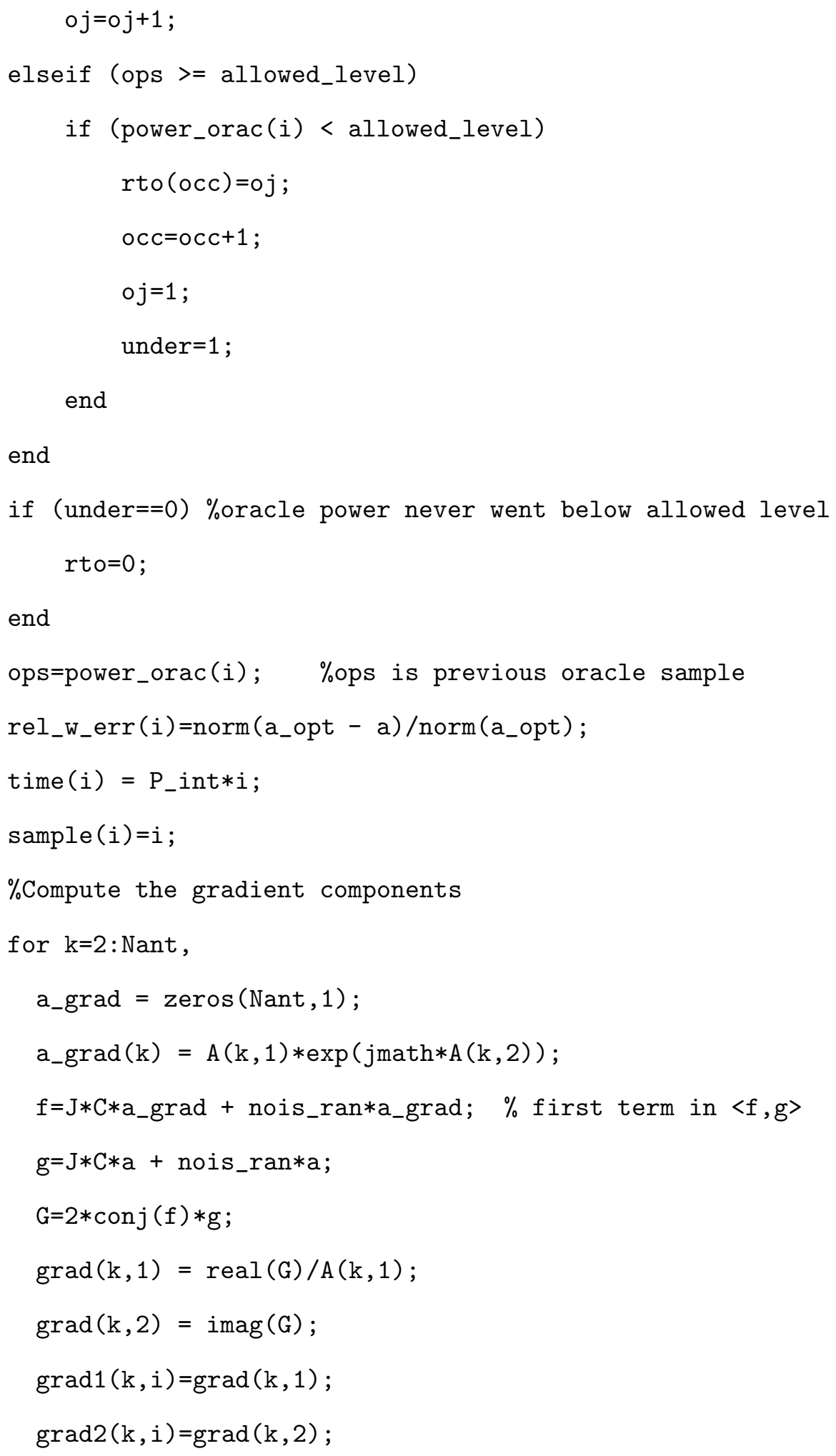


Appendix B (Continued)

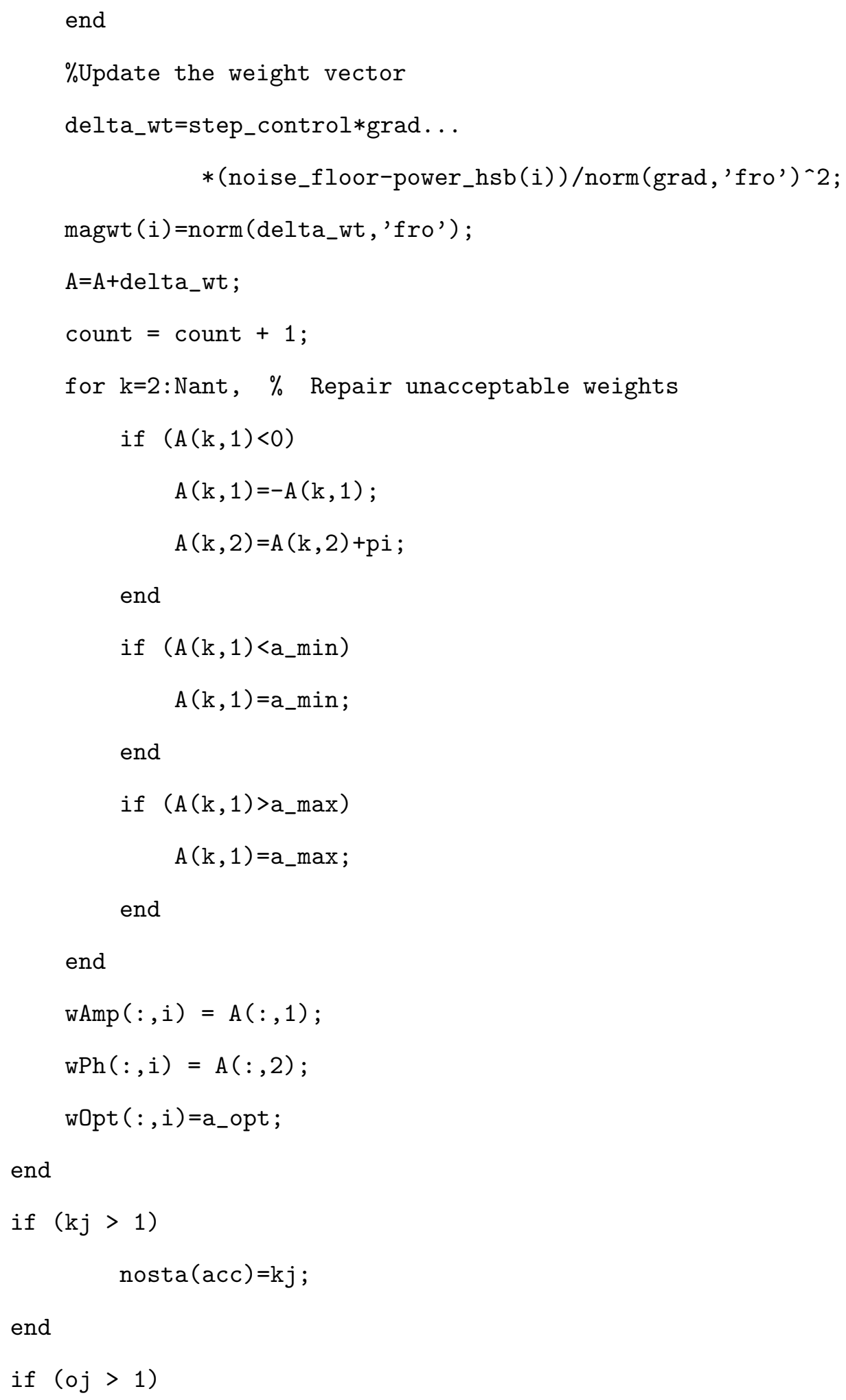


Appendix B (Continued)

$\operatorname{rto}(o c c)=o j$

end

for rows $=1: \operatorname{size}(w 0 p t, 1)$,

$\%$ change matrix of complex optimal weights to

for cols $=1: \operatorname{size}($ wOpt, 2$)$,

$\%$ matrix of magnitudes and matrix of phases

$\mathrm{mwO}($ rows, $\operatorname{cols})=\operatorname{sqrt}\left(\right.$ real $(\text { wOpt }(\text { rows }, \operatorname{cols}))^{\wedge} 2 \ldots$

+imag (wOpt (rows, cols) )^2);

$\mathrm{pwO}($ rows, cols $)=\operatorname{atan}($ imag $($ wOpt $($ rows, cols $)) \ldots$

/real (wOpt (rows, cols)));

end

end

\%Output power in $\mathrm{dB}$

power_orac_dB $=10 * \log 10$ (power_orac);

power_hsb_dB $=10 * \log 10$ (power_hsb);

$\%$ Failure rate (power $>$ allowed_level)

$\mathrm{b}=\mathrm{f}$ ind (power_hsb_dB $>=10 * \log 10($ allowed_level));

err=100*size(b)/size(power_hsb_dB);

orac_over=find (power_orac_dB $>=10 * \log 10($ allowed_level)) ;

err_orac $=100 * \operatorname{size}($ orac_over)/size (power_orac_dB);

\%Initial adaption iteration count

for $a b c=1:$ length (power_hsb_dB),

if $\operatorname{convg}==0$

its $=i t s+1$

end

if (power_hsb_dB $(a b c)<=10 * \log 10($ allowed_level))

convg $=1 ; \quad \%$ algorithm has reduced power below 
Appendix B (Continued)

end $\quad \%$ allowed level

end

its=its -1 ;

$\%$ Average re-convergence counts

if $(\mathrm{acc}>1)$

$\operatorname{str}=\operatorname{sum}($ nosta $) /(\operatorname{acc}-1)$;

else

str=0; \%power never went above allowed level

end

if $($ occ $>1)$

osc $=\operatorname{sum}($ rto $) /($ occ -1$) ; \quad \%$ oracle sample count

else

osc $=0 ; \%$ oracle never went above allowed level

end

$\%$ Plot output

figure;

subplot $(3,1,1)$

plot (time, power_hsb_dB)

legend('algorithm output power')

$\operatorname{axis}([0$ P_int*Niterations -1060$])$;

rep $=[$ num2str $(e r r)$, \% of time power exceeds allowed level '];

$\operatorname{text}(.001,55, \mathrm{rep})$;

rul $=[$ 'Average number of iterations to return below allowed level is '... num2str(round (str))];

$\operatorname{text}(.001,47, \mathrm{rul})$;

rots $=[$ num2str (roll/deg_to_rad) ' rollrate;' num2str (yaw/deg_to_rad)...

, yawrate; ' num2str(pitch/deg_to_rad) '... 
Appendix B (Continued)

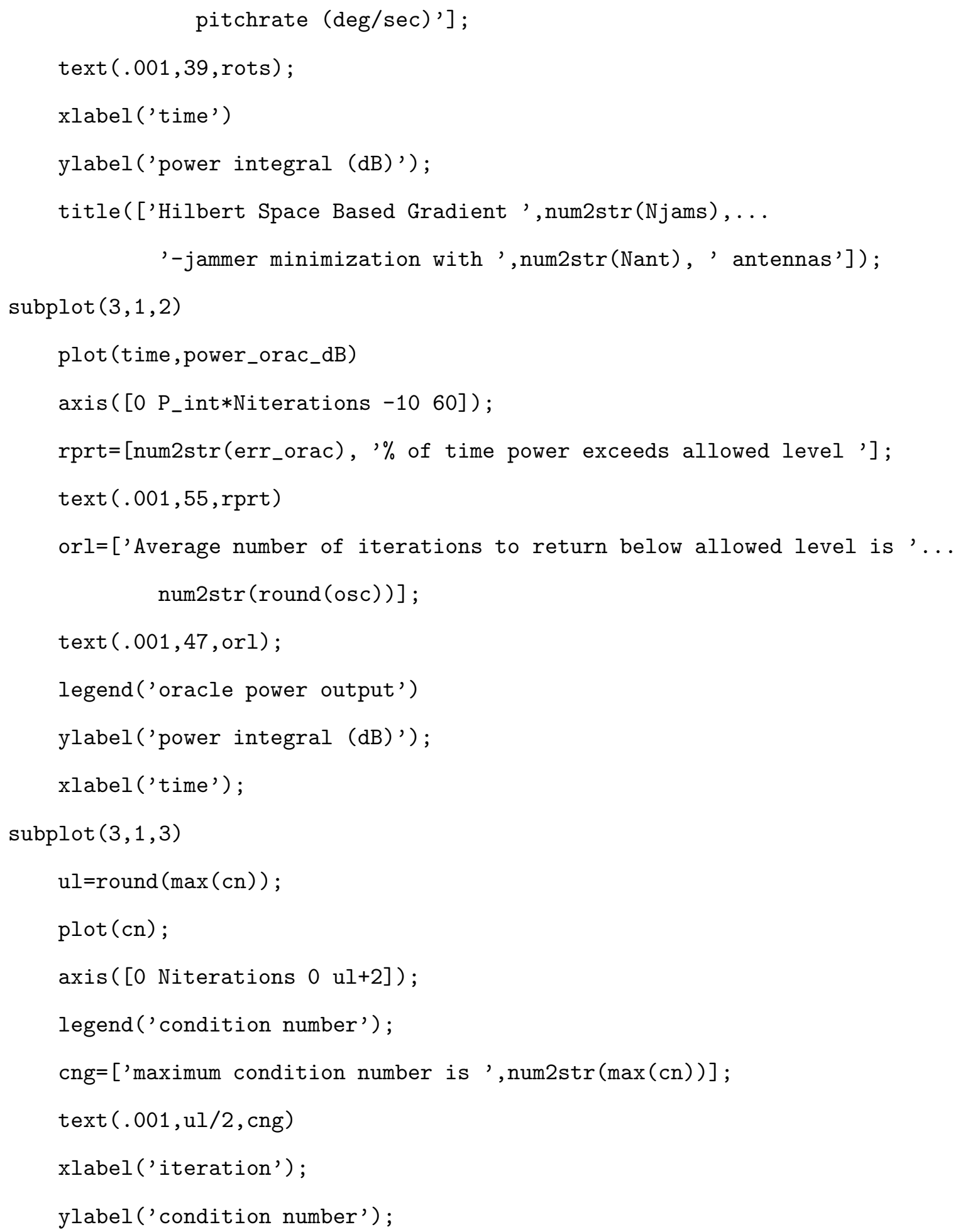


Appendix B (Continued)

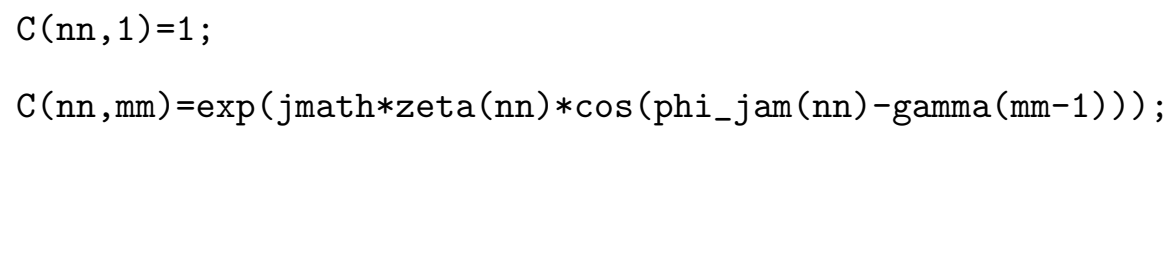




\section{Appendix C: GPS Signal Characteristics}

The Hilbert space based gradient algorithm is designed to adjust the weights of antennas in an array to detect signals from the Global Positioning System (GPS) Satellites while simultaneously nulling jammer signals. The GPS signals originate from one of 24 satellites. This network of satellites provides extremely accurate location and time data for navigational purposes. The signals transmitted from the satellites have very low power due to the limited fuel source onboard each satellite. The modulation scheme for the satellite signals is called Direct Sequence Spread Spectrum modulation. Spread spectrum modulates a narrowband signal into a wideband signal that appears to be random in nature while conserving the power in the signal. With the same amount of average power now over a much larger bandwidth, the instantaneous power of the signal is reduced. This is accomplished by modulating the data with a sequence of bits called chips that take on only two values +1 or -1 . The sequence of chips appears as random data but, in fact, is not. The sequence is called a pseudorandom or PN sequence because it can be generated from an equation, a primitive polynomial in $\mathrm{GF}(2)$. The sequence can be generated by implementing the primitive polynomial with a Linear Feedback Shift Register. For demodulation, the PN sequence that modulates the data must be reproduced at the receiver. Unintended receivers who do not know the PN sequence will not be able to demodulate the data. The receiver works by correlating the received signal with the locally generated PN sequence. The sequence has a high correlation with itself only at zero shift. When a high correlation is detected, the receiver integrates the signal over a data bit interval to recover the value of the data bit. This despreads the signal from wideband back to narrowband. The average power in the signal remains the same but the instantaneous power is increased due to the smaller bandwidth, thus improving correct bit detection [4], [13].

Each chip of the PN sequence is of fixed length, $T_{c}$ seconds, but this is much shorter than the length of a data bit, $T_{b}$ seconds. Modulating the data bits by this PN sequence increases the bandwidth or spreads the spectrum of the data signal from $B=1 / T_{b} \mathrm{~Hz}$ to 
Appendix C (Continued)

$W=1 / T_{c}$ Hz. The ratio $W / B$ is called the processing gain and is how much the bandwidth has increased due to spreading [14].

The nonspread GPS data rate is 50 bps BPSK, $T_{b}=1 / 50$ seconds. The transmitted, spread GPS signal consists of signals in quadrature. The I and Q components are spread spectrum modulated each with a distinct PN sequence. For the I component, the data is modulo-2 added to a PN sequence called the $\mathrm{P}$ (Precision) code and for the $\mathrm{Q}$ component, the data is modulo-2 added to another PN sequence called the C/A (Clear/Acquisition) code [7]. The chip length for the $\mathrm{P}$ code is $T_{c}=1 / 10.23 \times 10^{6}$ seconds giving a processing gain of

$$
\frac{W}{B}_{P}=\frac{T_{b}}{T_{c}}=204600
$$

Therefore, the spectral height of the I component is 204600 times lower than it would be if it were not spread. The chip length for the $\mathrm{C} / \mathrm{A}$ code is $T_{c}=1 / 1.023 \times 10^{6}$ seconds giving a processing gain of

$$
\frac{W}{B}_{C / A}=\frac{T_{b}}{T_{c}}=20460
$$

and so the spectral height of the Q component is 20460 times lower than it would be if it were not spread.

The ratio of signal energy per data bit, $\mathcal{E}_{b}=P_{a v} T_{b}$, to jammer power spectral density, $J_{0}=J_{a v} T_{c}$ is

$$
\frac{\mathcal{E}_{b}}{J_{0}}=\frac{P_{a v} T_{b}}{J_{a v} T_{c}}=\frac{T_{b} / T_{c}}{J_{a v} / P_{a v}}
$$

The ratio $\mathcal{E}_{b} / J_{0}$ can be interpreted as the SNR, while $T_{b} / T_{c}$ is the processing gain, and $J_{a v} / P_{a v}$ is the jamming margin [14]. If the processing gain and desired SNR level are known, the jamming margin from equation (40) represents the largest value a jammer can take with reference to the signal of interest for the system to still meet the desired SNR level. 
Appendix C (Continued)

As an example, a desired SNR level of $10.5 \mathrm{~dB}$ per bit or $10^{-6}$ error probability for a BPSK signal is chosen. The processing gain for the $\mathrm{P}$ code yields a jamming margin of

$$
10 \log _{10} \frac{J_{a v}}{P_{a v}}=10 \log _{10} \frac{T_{b}}{T_{c}}-S N R=10 \log _{10} 204600-10.5=42.61 \mathrm{~dB}
$$

For the $\mathrm{C} / \mathrm{A}$ code, the jamming margin is

$$
10 \log _{10} \frac{J_{a v}}{P_{a v}}=10 \log _{10} \frac{T_{b}}{T_{c}}-S N R=10 \log _{10} 20460-10.5=32.61 \mathrm{~dB}
$$

Given that the GPS signals received by a 0 dBIC antenna are on the order of -160 $\mathrm{dBw}[7]$ and the jamming margin of $32.61 \mathrm{~dB}$, the jammer signal cannot be stronger than $-160+32.61=-127.4 \mathrm{dBw}$ for this error probability to be achieved. This illustrates the advantage of using spread spectrum modulation in that the unwanted signal may actually be higher in power than the desired signal while still providing a low probability of bit error. This demonstrates that the goal of the algorithm to reduce the output power of the array to within $5 \mathrm{~dB}$ of the background noise level will allow a high probability of detection and demodulation of the GPS signals.

The signal processing required to obtain accurate navigational measurements from GPS signals is not relevant to this document. The interested reader will find a description of this in $[7]$. 


\section{Appendix D: Alternative Gradient Formula}

Dr. V. K. Jain has suggested the alternative derivation of the gradient formulas: Since

$$
\begin{aligned}
P(k) & =\langle x A, x A> \\
& =A^{H} x^{H} x A \\
& =A^{H} \Phi A
\end{aligned}
$$

is a real-valued function of $\mathrm{A}$,

$$
\begin{aligned}
\nabla_{A} P(k) & =2 \Phi A \\
& =2(F+i G) A
\end{aligned}
$$

where $\Phi$ is Hermitian symmetric: $F^{T}=F ; G^{T}=-G$. Also, if we let $A=a+i b$, then (since $P(k)$ is a real-valued function of $A$ )

$$
\nabla_{A}=\nabla_{a}+i \nabla_{b}
$$

Thus,

$$
\begin{aligned}
\nabla_{A} P & =2(F+i G)(a+i b) \\
& =2[(F a-G b)+i(F b+G a)]
\end{aligned}
$$

and

$$
\begin{aligned}
& \nabla_{a} P=2(F a-G b) \\
& \nabla_{b} P=2(F b+G a) .
\end{aligned}
$$

The gradient of the power with respect to the magnitude and phase of the antenna weights in terms of the gradient with respect to the real and imaginary parts of the antenna 
Appendix D (Continued)

weights can be found using the chain rule:

$$
\frac{\partial P}{\partial w}=\frac{\partial P}{\partial a} \frac{\partial a}{\partial w}+\frac{\partial P}{\partial b} \frac{\partial b}{\partial w}
$$

and

$$
\frac{\partial P}{\partial q}=\frac{\partial P}{\partial a} \frac{\partial a}{\partial q}+\frac{\partial P}{\partial b} \frac{\partial b}{\partial q}
$$

where $\frac{\partial a}{\partial w}=e^{i q}=\frac{A}{w}, \frac{\partial b}{\partial w}=-i e^{i q}=-i \frac{A}{w}, \frac{\partial a}{\partial q}=i w e^{i q}=i A$, and $\frac{\partial b}{\partial q}=w e^{i q}=A$. 


\begin{abstract}
About the Author
Sandra Gomulka Johnson was born in Johnstown, Pennsylvania. She attended the University of New Mexico in Albuquerque and obtained her BSEE in 1990. She attended graduate school at the University of Southern California in Los Angeles obtaining her MSEE in 1992. She was employed as a signal processing engineer at the Los Alamos National Laboratory, Los Alamos, New Mexico and at Raytheon, St. Petersburg, Florida before obtaining her $\mathrm{PhD}$ in Electrical Engineering at the University of South Florida in Tampa.
\end{abstract}

